\title{
A categorification of quantum sl(n)
}

\author{
Mikhail Khovanov and Aaron D. Lauda
}

\begin{abstract}
To an arbitrary root datum we associate a 2-category. For root datum corresponding to $\operatorname{sl}(n)$ we show that this 2-category categorifies the idempotented form of the quantum enveloping algebra.
\end{abstract}

Mathematics Subject Classification (2010). 81R50, 14M15, 17B37, 16W99.

Keywords. Categorification, quantum groups, quantum $\operatorname{sl}(n)$, iterated flag variety, 2-representation, 2-category

\section{Contents}

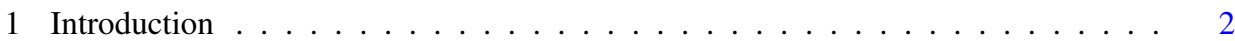

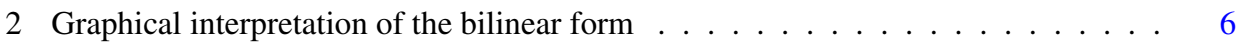

2.1 Quantum groups . . . . . . . . . . . . . . . . . 6

2.2 Geometric interpretation of the bilinear form . . . . . . . . . . . . . . 12

3 Graphical calculus for $\mathbf{U}$ categorification . . . . . . . . . . . . . . . . . . 21

3.1 The 2-category $u \ldots \ldots \ldots \ldots \ldots \ldots$

3.2 Spanning sets of HOMs in $u \ldots \ldots \ldots \ldots \ldots$

3.3 Properties and symmetries of 2-category $u \ldots \ldots \ldots \ldots$

3.4 Karoubi envelope, $\dot{u}$, and 2-representations . . . . . . . . . . . . . . . . . . . . 41

3.5 Direct sum decompositions . . . . . . . . . . . . . . . . . . . . . 42

$3.6 K_{0}(\dot{u})$ and homomorphism $\gamma \ldots \ldots \ldots \ldots \ldots$

3.7 Idempotented rings . . . . . . . . . . . . . . . . . . . . . . . 47

3.8 Surjectivity of $\gamma \ldots \ldots \ldots \ldots \ldots \ldots \ldots$

3.9 Injectivity of $\gamma$ in the non-degenerate case . . . . . . . . . . 56

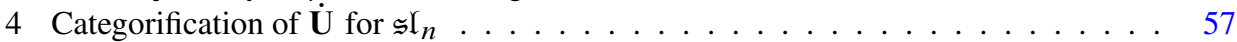

4.1 Forms of quantum $s_{n} \ldots \ldots \ldots \ldots$

4.2 The 2-category $u_{\rightarrow}\left(s_{n}\right) \ldots \ldots \ldots \ldots$

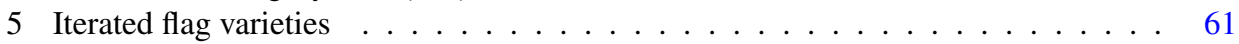

5.1 Cohomology of $n$-step varieties . . . . . . . . . . . . . . . . . 61

5.2 Graphical calculus for iterated flag varieties . . . . . . . . . . . . 66

5.3 The 2-category $\mathbf{F l a g}_{N} \ldots \ldots \ldots \ldots \ldots \ldots \ldots$

6 Representing $u_{\rightarrow}^{*}$ on the flag 2 -category . . . . . . . . . . . . . 76

6.1 Defining the 2 -functor $\Gamma_{N} \ldots \ldots \ldots \ldots \ldots \ldots$ 
6.2 Checking the relations of $u_{\rightarrow} \ldots \ldots \ldots \ldots \ldots$

6.3 Equivariant representation . . . . . . . . . . . . . . . . . . . . . 82

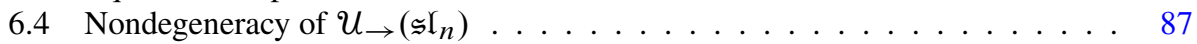

References . . . . . . . . . . . . . . . . . . . . 90

\section{Introduction}

In this paper we categorify the Beilinson-Lusztig-MacPherson idempotented modification $\dot{\mathbf{U}}\left(\mathfrak{s} \mathfrak{l}_{n}\right)$ of $\mathbf{U}_{q}\left(\mathfrak{s} \mathfrak{l}_{n}\right)$ for any $n$, generalizing [21], [22], where such categorification was described for $n=2$, and using constructions and results of [16], [17] which contain a categorification of $\mathbf{U}^{-}$for any Cartan datum. More generally, we define a 2-category associated to any root datum. The categorification of $\dot{\mathbf{U}}\left(\mathfrak{s} \mathfrak{l}_{n}\right)$ is given by the 2-category associated to the root system $A_{n-1}$.

In [24] Lusztig associates a quantum group $\mathbf{U}$ to any root datum; the latter consists of a perfect pairing $\langle$,$\rangle between two free abelian groups X$ and $Y$, embeddings of the set $I$ of simple roots into $X, Y$, and a bilinear form on $\mathbb{Z}[I]$ subject to certain compatibility and integrality conditions. Lusztig's definition is slightly different from the original ones due to Drinfeld [9] and Jimbo [13]. Lusztig then modifies $\mathbf{U}$ to the nonunital ring $\dot{\mathbf{U}}$ which contains a system of idempotents $\left\{1_{\lambda}\right\}$ over all weights $\lambda \in X$ as a substitute for the unit element,

$$
\dot{\mathbf{U}}=\bigoplus_{\lambda, \mu \in X} 1_{\mu} \dot{\mathbf{U}} 1_{\lambda}
$$

In the $\mathfrak{s l}_{n}$ case $\dot{\mathbf{U}}$ was originally defined by Beilinson, Lusztig, and Macpherson [2] and then appeared in [23], [14] in greater generality. It is clear from Lusztig's work that the $\mathbb{Q}(q)$-algebra $\dot{\mathbf{U}}$ is natural for at least the following reasons:

(1) A $\dot{\mathbf{U}}$-module is the same as a $\mathbf{U}$-module which has an integral weight decomposition. These modules are of prime importance in the representation theory of $\mathbf{U}$.

(2) $\dot{\mathbf{U}}$ has analogues of the comultiplication, the antipode, and other standard symmetries of $\mathbf{U}$.

(3) $\dot{\mathbf{U}}$ is a $\mathbf{U}$-bimodule.

(4) The Peter-Weyl theorem and the theory of cells can be intrinsically stated in terms of the algebra $\dot{\mathbf{U}}$.

(5) $\dot{\mathbf{U}}$ has an integral form ${ }_{\mathcal{A}} \dot{\mathbf{U}}$, a $\mathbb{Z}\left[q, q^{-1}\right]$-lattice closed under multiplication and comultiplication. The integral form comes with a canonical basis $\dot{\mathbf{B}}$. Conjecturally, multiplication and comultiplication in this basis have coefficients in $\mathbb{N}\left[q, q^{-1}\right]$ when the Cartan datum is symmetric.

(6) The braid group associated to the Cartan datum acts on $\dot{\mathbf{U}}$.

Moreover, $\dot{\mathbf{U}}$ appears throughout the categorification program for quantum groups. Representations of quantum groups that are known to have categorifications all have 
integral weight decompositions, and thus automatically extend to representations of $\dot{\mathbf{U}}$. In most or all of these examples, see [3], [7], [10], [31], [33], the weight decomposition of representations lifts to a direct sum decomposition of categories, so one obtains a categorification of the idempotent $1_{\lambda}$ as the functor of projection onto the corresponding direct summand (the only possible exception is the categorification of tensor products via the affine Grassmannian [5], [6]). In the categorification of tensor powers of the fundamental $\mathbf{U}_{q}\left(\mathfrak{s} \mathfrak{l}_{2}\right)$-representation [3], [10], each canonical basis element of $\dot{\mathbf{U}}$ acts as an indecomposable projective functor or as the zero functor. The idea that $\dot{\mathbf{U}}$ rather than $\mathbf{U}$ should be categorified goes back to Crane and Frenkel [8].

$\dot{\mathbf{U}}$ is generated by elements $E_{i} 1_{\lambda}, F_{i} 1_{\lambda}$, and $1_{\lambda}$, where $\lambda \in X$ is an element of the weight lattice and $i$ is a simple root. We will often write $E_{+i}$ instead of $E_{i}$ and $E_{-i}$ instead of $F_{i}$. We have

$$
E_{ \pm i} 1_{\lambda}=1_{\mu} E_{ \pm i} 1_{\lambda}
$$

where, in our notations, explained in Section 2.1, $\mu=\lambda \pm i_{X}$, and $i_{X}$ is the element of $X$ associated to the simple root $i$. Algebra $\dot{\mathbf{U}}$ is spanned by products

$$
E_{\boldsymbol{i}} 1_{\lambda}:=E_{ \pm i_{1}} E_{ \pm i_{2}} \ldots E_{ \pm i_{m}} 1_{\lambda}=1_{\mu} E_{ \pm i_{1}} E_{ \pm i_{2}} \ldots E_{ \pm i_{m}} 1_{\lambda}
$$

where $\boldsymbol{i}=\left( \pm i_{1}, \ldots, \pm i_{m}\right)$ is a signed sequence of simple roots, and $\mu=\lambda+\boldsymbol{i}_{X}$.

The integral form ${ }_{\mathcal{A}} \dot{\mathbf{U}} \subset \dot{\mathbf{U}}$ is the $\mathbb{Z}\left[q, q^{-1}\right]$-algebra generated by divided powers

$$
E_{i(a)} 1_{\lambda}=\frac{1}{[a]_{i} !} E_{i}^{a} 1_{\lambda} .
$$

Note that $\dot{\mathbf{U}}$ can, alternatively, be viewed as a pre-additive category with objects $\lambda \in X$ and morphisms from $\lambda$ to $\mu$ being $1_{\mu} \dot{\mathbf{U}} 1_{\lambda}$. Of course, any ring with a collection of mutually orthogonal idempotents as a substitute for the unit element can be viewed as a pre-additive category and vice versa. From this perspective, though, we can expect the categorification of $\dot{\mathbf{U}}$ to be a 2-category.

In Section 3.1 we associate a 2-category $U$ to a root datum. The objects of this 2-category are integral weights $\lambda \in X$, the morphisms from $\lambda$ to $\mu$ are finite formal sums of symbols $\mathcal{E}_{\boldsymbol{i}} \mathbf{1}_{\lambda}\{t\}$, where $\boldsymbol{i}=\left( \pm i_{1}, \ldots, \pm i_{m}\right)$ is a signed sequence of simple roots such that the left weight of the symbol is $\mu\left(E_{\boldsymbol{i}} 1_{\lambda}=1_{\mu} E_{\boldsymbol{i}} 1_{\lambda}\right)$, and $t \in \mathbb{Z}$ is a grading shift. When $\boldsymbol{i}$ consists of a single term, we get 1 -morphisms $\mathcal{E}_{+i} \mathbf{1}_{\lambda}$ and $\mathcal{E}_{-i} \mathbf{1}_{\lambda}$, which should be thought of as categorifying elements $E_{i} 1_{\lambda}$ and $F_{i} 1_{\lambda}$ of $\dot{\mathbf{U}}$, respectively. Grading shift $\{t\}$ categorifies multiplication by $q^{t}$. The 1 -morphism $\varepsilon_{i} \mathbf{1}_{\lambda}: \lambda \rightarrow \mu$ should be thought of as a categorification of the element $E_{\boldsymbol{i}} 1_{\lambda}$. When the sequence $\boldsymbol{i}$ is empty, we get the identity morphism $\mathbf{1}_{\lambda}: \lambda \rightarrow \lambda$, a categorification of the element $1_{\lambda}$.

Two-morphisms between $\mathcal{E}_{\boldsymbol{i}} \mathbf{1}_{\lambda}\{t\}$ and $\mathcal{E}_{\boldsymbol{j}} \mathbf{1}_{\lambda}\left\{t^{\prime}\right\}$ are given by linear combinations of degree $t-t^{\prime}$ diagrams drawn on the strip $\mathbb{R} \times[0,1]$ of the plane. The diagrams consist of immersed oriented one-manifolds, with every component labelled by a 
simple root, and dots placed on the components. Labels and orientations at the lower and upper endpoints of the one-manifold must match the sequences $\boldsymbol{i}$ and $\boldsymbol{j}$, respectively. Integral weights label regions of the plane cut out by the one-manifold, with the rightmost region labelled $\lambda$. Each diagram has an integer degree assigned to it. We work over a ground field $\mathbb{k}$, and define a 2-morphism between $\varepsilon_{\boldsymbol{i}} \mathbf{1}_{\lambda}\{t\}$ and $\mathscr{E}_{\boldsymbol{j}} \mathbf{1}_{\lambda}\left\{t^{\prime}\right\}$ as a linear combination of such diagrams of degree $t-t^{\prime}$, with coefficients in $\mathbb{k}$, modulo isotopies and a collection of very carefully chosen local relations. The set of 2-morphisms $U\left(\mathcal{E}_{\boldsymbol{i}} \mathbf{1}_{\lambda}\{t\}, \mathcal{E}_{\boldsymbol{j}} \mathbf{1}_{\lambda}\left\{t^{\prime}\right\}\right)$ is a $\mathbb{k}$-vector space. We also form graded vector space

$$
\operatorname{HOM}_{u}\left(\varepsilon_{\boldsymbol{i}} \mathbf{1}_{\lambda}, \varepsilon_{j} \mathbf{1}_{\lambda}\right):=\bigoplus_{t \in \mathbb{Z}} u\left(\varepsilon_{\boldsymbol{i}} \mathbf{1}_{\lambda}\{t\}, \varepsilon_{j} \mathbf{1}_{\lambda}\right)
$$

Vertical composition of 2-morphisms is given by concatenation of diagrams, horizontal composition consists of placing diagrams next to each other.

In each graded $\mathrm{k}$-vector space $\mathrm{HOM}_{\boldsymbol{u}}\left(\boldsymbol{E}_{\boldsymbol{i}} \mathbf{1}_{\lambda}, \boldsymbol{E}_{\boldsymbol{j}} \mathbf{1}_{\lambda}\right)$ we construct a homogeneous spanning set $B_{\boldsymbol{i}, \boldsymbol{j}, \lambda}$ which depends on extra choices. The Laurent power series in $q$, with the coefficient at $q^{r}$ equal to the number of spanning set elements of degree $r$, is proportional to suitably normalized inner product $\left\langle E_{\boldsymbol{i}} 1_{\lambda}, E_{\boldsymbol{j}} 1_{\lambda}\right\rangle$, where the semilinear form $\langle$,$\rangle is a mild modification of the Lusztig bilinear form on \dot{\mathbf{U}}$. The proportionality coefficient $\pi$ depends only on the root datum.

We say that our graphical calculus is non-degenerate for a given root datum and field $\mathrm{k}$ if for each $\boldsymbol{i}, \boldsymbol{j}$ and $\lambda$ the homogeneous spanning set $B_{\boldsymbol{i}, \boldsymbol{j}, \lambda}$ is a basis of the $k$-vector space $\operatorname{HOM}_{u}\left(\varepsilon_{\boldsymbol{i}} \mathbf{1}_{\lambda}, \mathcal{E}_{\boldsymbol{j}} \mathbf{1}_{\lambda}\right)$. Nondegeneracy will be crucial for our categorification constructions.

The 2-category $\mathcal{U}$ is $\mathbb{k}$-additive, and we form its Karoubian envelope $\dot{U}$, the smallest 2-category which contains $\mathcal{U}$ and has splitting idempotents. Namely, for each $\lambda, \mu \in X$, the category $\dot{U}(\lambda, \mu)$ of morphisms $\lambda \rightarrow \mu$ is defined as the Karoubian envelope of the additive $\mathbb{k}$-linear category $U(\lambda, \mu)$. The split Grothendieck category $K_{0}(\dot{U})$ is a pre-additive category with objects $\lambda$, and the abelian group of morphisms from $\lambda$ to $\mu$ is the split Grothendieck group $K_{0}(\dot{U}(\lambda, \mu))$ of the additive category $\dot{U}(\lambda, \mu))$. The grading shift functor on $\dot{U}(\lambda, \mu)$ turns $K_{0}(\dot{U}(\lambda, \mu))$ into a $\mathbb{Z}\left[q, q^{-1}\right]$-module. This module is free with the basis given by isomorphism classes of indecomposable objects of $\dot{U}(\lambda, \mu)$, up to grading shifts. The split Grothendieck category $K_{0}(\dot{U})$ can also be viewed as a nonunital $\mathbb{Z}\left[q, q^{-1}\right]$-algebra with a collection of idempotents $\left[\mathbf{1}_{\lambda}\right]$ as a substitute for the unit element.

In Section 3.6 we set up a $\mathbb{Z}\left[q, q^{-1}\right]$-algebra homomorphism

$$
\gamma:{ }_{A} \dot{\mathbf{U}} \rightarrow K_{0}(\dot{U})
$$

which takes $1_{\lambda}$ to $\left[\mathbf{1}_{\lambda}\right]$ and $E_{\boldsymbol{i}} \mathbf{1}_{\lambda}$ to $\left[\mathcal{E}_{\boldsymbol{i}} \mathbf{1}_{\lambda}\right]$, for any "divided power" signed sequence $\boldsymbol{i}$.

The main results of this paper are the following theorems.

Theorem 1.1. The map $\gamma$ is surjective for any root datum and field $\mathrm{k}$. 
Theorem 1.2. The map $\gamma$ is injective if the graphical calculus for the root datum and field $\mathrm{k}$ is non-degenerate.

Theorem 1.3. The graphical calculus is non-degenerate for the root datum of $\mathfrak{I}_{n}$ and any field $\mathrm{k}$.

The three theorems together immediately imply

Proposition 1.4. The map $\gamma$ is an isomorphism for the root datum of $\mathfrak{s l}_{n}$ and any field $\mathrm{k}$.

The last result establishes a canonical isomorphism

$$
{ }_{\mathcal{A}} \dot{\mathbf{U}}\left(\mathfrak{s} \mathfrak{l}_{n}\right) \cong K_{0}\left(\dot{U}\left(\mathfrak{s} \mathfrak{l}_{n}\right)\right)
$$

and allows us to view $\dot{U}\left(\mathfrak{s} \mathfrak{l}_{n}\right)$ as a categorification of $\dot{\mathbf{U}}\left(\mathfrak{s} \mathfrak{l}_{n}\right)$.

Theorem 1.1, proved in Section 3.8, follows from the results of [16], [17], [21] and basic properties of Grothendieck groups and idempotents. Theorem 1.2, proved in Section 3.9, follows from the non-degeneracy of the semilinear form on $\dot{\mathbf{U}}$ and its pictorial interpretation explained in Section 2.2. To prove theorem 1.3 we construct a family of 2-representations of $\dot{U}$ and check that the elements of each spanning set $B_{\boldsymbol{i}, \boldsymbol{j}, \lambda}$ act linearly independently on vector spaces in these 2-representations, implying non-degeneracy of the graphical calculus. Sections 4-6 are devoted to these constructions.

Indecomposable 1-morphisms, up to isomorphism and grading shifts, constitute a basis of $K_{0}\left(\dot{U}\left(\mathfrak{s} \mathfrak{l}_{n}\right)\right) \cong{ }_{A} \dot{U}\left(\mathfrak{s} \mathfrak{l}_{n}\right)$, which might potentially depend on the ground field $\mathbb{k}$. The multiplication in this basis has coefficients in $\mathbb{N}\left[q, q^{-1}\right]$. It is an open problem whether this basis coincides with the Lusztig canonical basis of ${ }_{\mathcal{A}} \dot{\mathbf{U}}\left(\mathfrak{s} \mathfrak{l}_{n}\right)$. The answer is positive when $n=2$, see [21].

Another major problem is to determine for which root data the graphical calculus is non-degenerate. Nondegeneracy immediately implies, via Theorems 1.1 and 1.2, that $\dot{U}$ categorifies $\dot{\mathbf{U}}$ for a given root datum.

We believe that $\dot{U}$ will prove ubiquitous in representation theory. This 2-category or its mild modifications is expected to act on parabolic-singular blocks of highest weight categories for $\mathfrak{s} \mathfrak{l}_{N}$ in the context of categorification of $\mathfrak{s} \mathfrak{l}_{n}$ representations [7], [10], [31], on derived categories of coherent sheaves on Kronheimer-Nakajima [20] and Nakajima [26] quiver varieties and on their Fukaya-Floer counterparts, on categories of modules over cyclotomic Hecke and degenerate Hecke algebras [1], [19], on categories of perverse sheaves in Zheng's categorifications of tensor products [33], on categories of modules over cyclotomic quotients of rings $R(v)$ in [16], [17], on categories of matrix factorizations that appear in [18, Section 11], etc. A possible approach to proving that the calculus is non-degenerate for other root systems is to show that $\dot{U}$ acts on a sufficiently large 2 -category and verify that the spanning 
set elements act linearly independently. It would also be interesting to relate our constructions with those of Rouquier [29], [30].

Categories of projective modules over rings $R(v)$, defined in [16], [17], categorify $\mathbf{U}^{-}$weight spaces. A subset of our defining local relations on 2-morphisms gives the relations for rings $R(v)$. This subset consists exactly of the relations whose diagrams have no critical points (U-turns) on strands and have all strand orientations going in the same direction. In other words, the relations on braid-like diagrams allow us to categorify $\mathbf{U}^{-}$, while the relations without these restrictions lead to a categorification of the entire $\dot{\mathbf{U}}$, at least in the $\mathfrak{s} \mathfrak{l}_{n}$ case. Informally, the passage from a categorification of $\mathbf{U}^{-}$to a categorification of $\dot{\mathbf{U}}$ is analogous to generalizing from braids to tangles.

Acknowledgments. M. K. was partially supported by the NSF grant DMS-0706924 and, during the early stages of this work, by the Institute for Advanced Study.

\section{Graphical interpretation of the bilinear form}

\subsection{Quantum groups}

2.1.1. Algebras f and $\mathbf{U}$. We recall several definitions, following [24]. A Cartan $\operatorname{datum}(I, \cdot)$ consists of a finite set $I$ and a symmetric $\mathbb{Z}$-valued bilinear form on $\mathbb{Z}[I]$, subject to conditions

- $i \cdot i \in\{2,4,6, \ldots\}$ for $i \in I$,

- $d_{i j}:=-2 \frac{i \cdot j}{i \cdot i} \in\{0,1,2, \ldots\}$ for any $i \neq j$ in $I$.

Let $q_{i}=q^{\frac{i \cdot i}{2}},[a]_{i}=q_{i}^{a-1}+q_{i}^{a-3}+\cdots+q_{i}^{1-a},[a]_{i} !=[a]_{i}[a-1]_{i} \ldots[1]_{i}$. Denote by 'f the free associative algebra over $\mathbb{Q}(q)$ with generators $\theta_{i}, i \in I$, and introduce $q$-divided powers $\theta_{i}^{(a)}=\theta_{i}^{a} /[a]_{i}$ !. The algebra'f is $\mathbb{N}[I]$-graded, with $\theta_{i}$ in degree $i$. The tensor square' $\mathbf{f} \otimes^{\prime} \mathbf{f}$ is an associative algebra with twisted multiplication

$$
\left(x_{1} \otimes x_{2}\right)\left(x_{1}^{\prime} \otimes x_{2}^{\prime}\right)=q^{-\left|x_{2}\right| \cdot\left|x_{1}^{\prime}\right|} x_{1} x_{1}^{\prime} \otimes x_{2} x_{2}^{\prime}
$$

for homogeneous $x_{1}, x_{2}, x_{1}^{\prime}, x_{2}^{\prime}$. The assignment $r\left(\theta_{i}\right)=\theta_{i} \otimes 1+1 \otimes \theta_{i}$ extends to a unique algebra homomorphism $r:{ }^{\prime} \mathbf{f} \rightarrow{ }^{\prime} \mathbf{f} \otimes^{\prime} \mathbf{f}$.

The algebra' $\mathbf{f}$ carries a $\mathbb{Q}(q)$-bilinear form determined by the conditions ${ }^{1}$

- $(1,1)=1$,

- $\left(\theta_{i}, \theta_{j}\right)=\delta_{i, j}\left(1-q_{i}^{2}\right)^{-1}$ for $i, j \in I$,

- $\left(x, y y^{\prime}\right)=\left(r(x), y \otimes y^{\prime}\right)$ for $x, y, y^{\prime} \in{ }^{\prime} \mathbf{f}$,

- $\left(x x^{\prime}, y\right)=\left(x \otimes x^{\prime}, r(y)\right)$ for $x, x^{\prime}, y \in{ }^{\prime} \mathbf{f}$.

The bilinear form $($,$) is symmetric. Its radical \mathfrak{s}$ is a two-sided ideal of ${ }^{\prime} \mathbf{f}$. The form (, ) descends to a non-degenerate form on the associative $\mathbb{Q}(q)$-algebra $\mathbf{f}=$ 'f $/ \mathfrak{S}$.

\footnotetext{
${ }^{1}$ Our bilinear form ( , ) corresponds to Lusztig's bilinear form $\{$, $\}$, see Lusztig [24, 1.2.10].
} 
Theorem 2.1. The ideal $\mathfrak{s}$ is generated by the elements

$$
\sum_{a+b=d_{i j}+1}(-1)^{a} \theta_{i}^{(a)} \theta_{j} \theta_{i}^{(b)}
$$

over all $i, j \in I, i \neq j$.

It seems that the only known proof of this theorem, for a general Cartan datum, requires Lusztig's geometric realization of $\mathbf{f}$ via perverse sheaves. This proof is given in his book [24, Theorem 33.1.3]. Less sophisticated proofs exist when the Cartan datum is finite.

We see that $\mathbf{f}$ is the quotient of 'f by the quantum Serre relations

$$
\sum_{a+b=d_{i j}+1}(-1)^{a} \theta_{i}^{(a)} \theta_{j} \theta_{i}^{(b)}=0
$$

Let ${ }_{\mathcal{A}} \mathbf{f}$ be the $\mathbb{Z}\left[q, q^{-1}\right]$-subalgebra of $\mathbf{f}$ generated by the divided powers $\theta_{i}^{(a)}$, over all $i \in I$ and $a \in \mathbb{N}$.

A root datum of type $(I, \cdot)$ consists of

- free finitely generated abelian groups $X, Y$ and a perfect pairing $\langle\rangle:, Y \times X \rightarrow$ $\mathbb{Z}$;

- inclusions $I \subset X\left(i \mapsto i_{X}\right)$ and $I \subset Y(i \mapsto i)$ such that $\left\langle i, j_{X}\right\rangle=2 \frac{i \cdot j}{i \cdot i}=-d_{i j}$ for all $i, j \in I$.

This implies $\left\langle i, i_{X}\right\rangle=2$ for all $i$. We write $i_{X}$, rather than Lusztig's $i^{\prime}$, to denote the image of $i$ in $X$.

The quantum group $\mathbf{U}$ associated to a root datum as above is the unital associative $\mathbb{Q}(q)$-algebra given by generators $E_{i}, F_{i}, K_{\mu}$ for $i \in I$ and $\mu \in Y$, subject to the relations

i) $K_{0}=1, K_{\mu} K_{\mu^{\prime}}=K_{\mu+\mu^{\prime}}$ for all $\mu, \mu^{\prime} \in Y$,

ii) $K_{\mu} E_{i}=q^{\left\langle\mu, i_{X}\right\rangle} E_{i} K_{\mu}$ for all $i \in I, \mu \in Y$,

iii) $K_{\mu} F_{i}=q^{-\left\langle\mu, i_{X}\right\rangle} F_{i} K_{\mu}$ for all $i \in I, \mu \in Y$,

iv) $E_{i} F_{j}-F_{j} E_{i}=\delta_{i j} \frac{\tilde{K}_{i}-\tilde{K}_{-i}}{q_{i}-q_{i}^{-1}}$, where $\tilde{K}_{ \pm i}=K_{ \pm(i \cdot i / 2) i}$,

v) for all $i \neq j$

$$
\sum_{a+b=-\left\langle i, j_{X}\right\rangle+1}(-1)^{a} E_{i}^{(a)} E_{j} E_{i}^{(b)}=0 \quad \text { and } \sum_{a+b=-\left\langle i, j_{X}\right\rangle+1}(-1)^{a} F_{i}^{(a)} F_{j} F_{i}^{(b)}=0 .
$$

If $f\left(\theta_{i}\right) \in \mathfrak{I}$ for a polynomial $f$ in noncommutative variables $\theta_{i}, i \in I$, then $f\left(E_{i}\right)=0$ and $f\left(F_{i}\right)=0$ in $\mathbf{U}$. This gives a pair of injective algebra homomorphisms $\mathbf{f} \rightarrow \mathbf{U}$.

2.1.2. Some automorphisms of $\mathbf{U}$. Let ${ }^{-}$be the $\mathbb{Q}$-linear involution of $\mathbb{Q}(q)$ which maps $q$ to $q^{-1}$. $\mathbf{U}$ has the following standard algebra (anti)automorphisms: 
- the $\mathbb{Q}(q)$-antilinear algebra involution $\psi: \mathbf{U} \rightarrow \mathbf{U}$ given by

$$
\psi\left(E_{i}\right)=E_{i}, \quad \psi\left(F_{i}\right)=F_{i}, \quad \psi\left(K_{\mu}\right)=K_{-\mu}, \quad \psi(f x)=\bar{f} \psi(x)
$$

for $f \in \mathbb{Q}(q)$ and $x \in \mathbf{U}$;

- the $\mathbb{Q}(q)$-linear algebra involution $\omega: \mathbf{U} \rightarrow \mathbf{U}$ given by

$$
\omega\left(E_{i}\right)=F_{i}, \quad \omega\left(F_{i}\right)=E_{i}, \quad \omega\left(K_{\mu}\right)=K_{-\mu} ;
$$

- the $\mathbb{Q}(q)$-linear algebra antiinvolution $\sigma: \mathbf{U} \rightarrow \mathbf{U}$ given by

$$
\sigma\left(E_{i}\right)=E_{i}, \quad \sigma\left(F_{i}\right)=F_{i}, \quad \sigma\left(K_{\mu}\right)=K_{\mu}
$$

- the $\mathbb{Q}(q)$-linear algebra antiinvolution $\rho: \mathbf{U} \rightarrow \mathbf{U}$ given by

$$
\rho\left(E_{i}\right)=q_{i} \tilde{K}_{i} F_{i}, \quad \rho\left(F_{i}\right)=q_{i} \tilde{K}_{-i} E_{i}, \quad \rho\left(K_{\mu}\right)=K_{\mu},
$$

we denote by $\bar{\rho}$ the $\mathbb{Q}(q)$-linear antiinvolution $\psi \rho \psi: \mathbf{U} \rightarrow \mathbf{U}$;

- the $\mathbb{Q}(q)$-antilinear antiautomorphism $\tau: \mathbf{U} \rightarrow \mathbf{U}$ defined as the composite $\tau=\psi \rho$,

$$
\tau\left(E_{i}\right)=q_{i}^{-1} \tilde{K}_{-i} F_{i}, \quad \tau\left(F_{i}\right)=q_{i}^{-1} \tilde{K}_{i} E_{i}, \quad \tau\left(K_{\mu}\right)=K_{-\mu} .
$$

2.1.3. $\dot{\mathbf{U}}$ and the bilinear form. The $\mathbb{Q}(q)$-algebra $\dot{\mathbf{U}}$ is obtained from $\mathbf{U}$ by adjoining a collection of orthogonal idempotents $1_{\lambda}$ for each $\lambda \in X$,

$$
1_{\lambda} 1_{\lambda^{\prime}}=\delta_{\lambda, \lambda^{\prime}} 1_{\lambda^{\prime}}
$$

such that

$$
K_{\mu} 1_{\lambda}=1_{\lambda} K_{\mu}=q^{\langle\mu, \lambda\rangle} 1_{\lambda}, \quad E_{i} 1_{\lambda}=1_{\lambda+i_{X}} E_{i}, \quad F_{i} 1_{\lambda}=1_{\lambda-i_{X}} F_{i} .
$$

The algebra $\dot{\mathbf{U}}$ decomposes as direct sum of weight spaces

$$
\dot{\mathbf{U}}=\bigoplus_{\lambda, \lambda^{\prime} \in X} 1_{\lambda^{\prime}} \dot{\mathbf{U}} 1_{\lambda}
$$

We say that $\lambda$, respectively $\lambda^{\prime}$, is the right, respectively left, weight of $x \in 1_{\lambda^{\prime}} \mathbf{U} 1_{\lambda}$. The algebra ${ }_{\mathcal{A}} \dot{\mathbf{U}}$ is the $\mathbb{Z}\left[q, q^{-1}\right]$-subalgebra of $\dot{\mathbf{U}}$ generated by products of divided powers $E_{i}^{(a)} 1_{\lambda}$ and $F_{i}^{(a)} 1_{\lambda}$, and has a similar weight decomposition

$$
{ }_{\mathcal{A}} \dot{\mathbf{U}}=\bigoplus_{\lambda, \lambda^{\prime} \in X} 1_{\lambda^{\prime}}\left({ }_{\mathcal{A}} \dot{\mathbf{U}}\right) 1_{\lambda} .
$$


The following identities hold in $\dot{\mathbf{U}}$ and ${ }_{\mathcal{A}} \dot{\mathbf{U}}$ :

$$
\begin{aligned}
& \left(E_{i} F_{j}-F_{j} E_{i}\right) 1_{\lambda}=\delta_{i, j}[\langle i, \lambda\rangle]_{i} 1_{\lambda}, \\
& E_{i}^{(a)} 1_{\lambda}=1_{\lambda+a i_{X}} E_{i}^{(a)}, \quad F_{i}^{(a)} 1_{\lambda}=1_{\lambda-a i_{X}} F_{i}^{(a)}, \\
& E_{i}^{(a)} F_{i}^{(b)} 1_{\lambda}=\sum_{t=0}^{\min (a, b)}\left[\begin{array}{c}
a-b+\langle i, \lambda\rangle \\
t
\end{array}\right]_{i} F_{i}^{(b-t)} E_{i}^{(a-t)} 1_{\lambda}, \\
& F_{i}^{(b)} E_{i}^{(a)} 1_{\lambda}=\sum_{t=0}^{\min (a, b)}\left[\begin{array}{c}
-a+b-\langle i, \lambda\rangle \\
t
\end{array}\right]_{i} E_{i}^{(a-t)} F_{i}^{(b-t)} 1_{\lambda} .
\end{aligned}
$$

The (anti) automorphisms $\psi, \omega, \sigma, \rho$ and $\tau$ all naturally extend to $\dot{\mathbf{U}}$ and ${ }_{\mathcal{A}} \dot{\mathbf{U}}$ if we set

$$
\psi\left(1_{\lambda}\right)=1_{\lambda}, \quad \omega\left(1_{\lambda}\right)=1_{-\lambda}, \quad \sigma\left(1_{\lambda}\right)=1_{-\lambda}, \quad \rho\left(1_{\lambda}\right)=1_{\lambda}, \quad \tau\left(1_{\lambda}\right)=1_{\lambda} .
$$

Taking direct sums of the induced maps on each summand $1_{\lambda^{\prime}} \dot{\mathbf{U}} 1_{\lambda}$ allows these maps to be extended to $\dot{\mathbf{U}}$. For example, the antiautomorphism $\tau$ induces for each $\lambda$ and $\lambda^{\prime}$ in $X$ an isomorphism $1_{\lambda^{\prime}} \dot{\mathbf{U}} 1_{\lambda} \rightarrow 1_{\lambda} \dot{\mathbf{U}} 1_{\lambda^{\prime}}$. Restricting to the $\mathbb{Z}\left[q, q^{-1}\right]$-subalgebra ${ }_{\mathcal{A}} \dot{\mathbf{U}}$ and taking direct sums, we obtain an algebra antiautomorphism $\tau:{ }_{\mathcal{A}} \dot{\mathbf{U}} \rightarrow{ }_{\mathcal{A}} \dot{\mathbf{U}}$ such that $\tau\left(1_{\lambda}\right)=1_{\lambda}, \tau\left(1_{\lambda+i_{X}} E_{i} 1_{\lambda}\right)=q_{i}^{-1-\langle i, \lambda\rangle} 1_{\lambda} F_{i} 1_{\lambda+i_{X}}$, and $\tau\left(1_{\lambda} F_{i} 1_{\lambda+i_{X}}\right)=$ $q_{i}^{1+\langle i, \lambda\rangle} 1_{\lambda+i_{X}} E_{i} 1_{\lambda}$ for all $\lambda \in X$.

The following result is taken from Lusztig [24, 26.1.1], but our bilinear form is normalized slightly differently from his.

Proposition 2.2. There exists a unique pairing $():, \dot{\mathbf{U}} \times \dot{\mathbf{U}} \rightarrow \mathbb{Q}(q)$ with the properties:

(i) (, ) is bilinear, i.e., $(f x, y)=f(x, y),(x, f y)=f(x, y)$ for $f \in \mathbb{Q}(q)$ and $x, y \in \dot{\mathbf{U}}$,

(ii) $\left(1_{\lambda_{1}} x 1_{\lambda_{2}}, 1_{\lambda_{1}^{\prime}} y 1_{\lambda_{2}^{\prime}}\right)=0$ for all $x, y \in \dot{\mathbf{U}}$ unless $\lambda_{1}=\lambda_{1}^{\prime}$ and $\lambda_{2}=\lambda_{2}^{\prime}$,

(iii) $(u x, y)=(x, \bar{\rho}(u) y)$ for $u \in \mathbf{U}$ and $x, y \in \dot{\mathbf{U}}$,

(iv) $\left(x 1_{\lambda}, x^{\prime} 1_{\lambda}\right)=\left(x, x^{\prime}\right)$ for all $x, x^{\prime} \in \mathbf{f} \cong \mathbf{U}^{+}$and all $\lambda$ (here $\left(x, x^{\prime}\right)$ is as in Section 2.1.1),

(v) $(x, y)=(y, x)$ for all $x, y \in \dot{\mathbf{U}}$.

Definition 2.3. Define a semilinear form $\langle\rangle:, \dot{\mathbf{U}} \times \dot{\mathbf{U}} \rightarrow \mathbb{Q}(q)$ by

$$
\langle x, y\rangle:=(\psi(x), y) \text { for all } x, y \in \dot{\mathbf{U}}
$$

Proposition 2.4. The map $\langle\rangle:, \dot{\mathbf{U}} \times \dot{\mathbf{U}} \rightarrow \mathbb{Q}(q)$ has the following properties:

(i) $\langle$, $\rangle$ is semilinear, i.e., $\langle f x, y\rangle=\bar{f}\langle x, y\rangle,\langle x, f y\rangle=f\langle x, y\rangle$ for $f \in \mathbb{Q}(q)$ and $x, y \in \dot{\mathbf{U}}$, 
(ii) $\left\langle 1_{\lambda_{1}} x 1_{\lambda_{2}}, 1_{\lambda_{1}^{\prime}} y 1_{\lambda_{2}^{\prime}}\right\rangle=0$ for all $x, y \in \dot{\mathbf{U}}$ unless $\lambda_{1}=\lambda_{1}^{\prime}$ and $\lambda_{2}=\lambda_{2}^{\prime}$,

(iii) $\langle u x, y\rangle=\langle x, \tau(u) y\rangle$ for $u \in \mathbf{U}$ and $x, y \in \dot{\mathbf{U}}$,

(iv) $\left\langle x 1_{\lambda}, x^{\prime} 1_{\lambda}\right\rangle=\left(\psi(x), x^{\prime}\right)$ for $x, x^{\prime} \in \mathbf{f}$ and $\lambda \in X$,

(v) $\langle x, y\rangle=\langle\psi(y), \psi(x)\rangle$ for all $x, y \in \dot{\mathbf{U}}$.

Proof. Immediate.

Proposition 2.5. The bilinear form (, ) and the semilinear form $\langle$,$\rangle are both non-$ degenerate on $\dot{\mathbf{U}}$.

Proof. Nondegeneracy of (, ) is implicit throughout [24, Chapter 26] and follows for instance from Theorem 26.3.1 of [24].

The bilinear and semilinear forms restrict to pairings

$$
(,):{ }_{\mathcal{A}} \dot{\mathbf{U}} \times{ }_{\mathcal{A}} \dot{\mathbf{U}} \rightarrow \mathbb{Z}\left[q, q^{-1}\right], \quad\langle,\rangle:{ }_{\mathcal{A}} \dot{\mathbf{U}} \times{ }_{\mathcal{A}} \dot{\mathbf{U}} \rightarrow \mathbb{Z}\left[q, q^{-1}\right] .
$$

2.1.4. Signed sequences. Throughout the paper we write $E_{+i}$ for $E_{i}$ and $E_{-i}$ for $F_{i}$ and need notation for products of these elements. By a signed sequence $\boldsymbol{i}=$ $\left(\varepsilon_{1} i_{1}, \varepsilon_{2} i_{2}, \ldots, \varepsilon_{m} i_{m}\right)$, where $\varepsilon_{1}, \ldots, \varepsilon_{m} \in\{+,-\}$ and $i_{1}, \ldots, i_{m} \in I$, we mean a finite sequence of elements of $I$ with signs. We may also write $i$ as $\varepsilon_{1} i_{1}, \varepsilon_{2} i_{2}, \ldots, \varepsilon_{m} i_{m}$ or even $\varepsilon_{1} i_{1} \varepsilon_{2} i_{2} \ldots \varepsilon_{m} i_{m}$. Let $|i|=\sum_{k=1}^{m} \varepsilon_{k} i_{k}$, viewed as an element of $\mathbb{Z}[I]$. The length of $\boldsymbol{i}$, denoted $\|\boldsymbol{i}\|$, is the number of elements in the sequence $\boldsymbol{i}$ ( $m$ in the above notations). Define SSeq to be the set of signed sequences. Let

$$
\begin{aligned}
E_{\boldsymbol{i}} & :=E_{\varepsilon_{1} i_{1}} E_{\varepsilon_{2} i_{2}} \ldots E_{\varepsilon_{m} i_{m}} \in \mathbf{U}, \\
E_{\boldsymbol{i}} 1_{\lambda} & :=E_{\varepsilon_{1} i_{1}} E_{\varepsilon_{2} i_{2}} \ldots E_{\varepsilon_{m} i_{m}} 1_{\lambda} \in \dot{\mathbf{U}} .
\end{aligned}
$$

Recall that $i_{X} \in X$ denotes the image of $i$ under the embedding $I \rightarrow X$. Let

$$
\boldsymbol{i}_{X}:=\varepsilon_{1}\left(i_{1}\right)_{X}+\cdots+\varepsilon_{m}\left(i_{m}\right)_{X} \in X .
$$

Then $E_{\boldsymbol{i}} 1_{\lambda}=1_{\lambda+\boldsymbol{i}_{X}} E_{\boldsymbol{i}} 1_{\lambda}$, so that $\lambda$, respectively $\lambda+\boldsymbol{i}_{X}$, is the right, respectively left, weight of $E_{\boldsymbol{i}} 1_{\lambda}$.

A sequence $\boldsymbol{i}$ is positive if all signs $\varepsilon_{1}, \ldots, \varepsilon_{m}=+$. We may write a positive sequence as $\left(i_{1}, \ldots, i_{m}\right)$ or even $i_{1} \ldots i_{m}$. Denote by SSeq ${ }^{+}$the set of all positive sequences. As in [16], for $v \in \mathbb{N}[I], v=\sum v_{i} \cdot i$; denote by $\operatorname{Seq}(v)$ the set of all sequences $\boldsymbol{i}=i_{1} \ldots i_{m}$ such that $i$ appears $v_{i}$ times in the sequence. There is an obvious bijection

$$
\mathrm{SSeq}^{+} \cong \coprod_{\nu \in \mathbb{N}[I]} \operatorname{Seq}(v) .
$$

A sequence is negative if all signs $\varepsilon_{1}, \ldots, \varepsilon_{m}=-$. For a positive sequence $\boldsymbol{i}$ denote by $-\boldsymbol{i}$ the corresponding negative sequence. For any signed sequence $\boldsymbol{i}$ denote by $-\boldsymbol{i}$ 
the sequence given by reversing all signs in $\boldsymbol{i}$. We write concatenation of sequences $\boldsymbol{i}$ and $\boldsymbol{j}$ as $\boldsymbol{i} \boldsymbol{j}$, attaching $\pm i$ to the left of $\boldsymbol{i}$ as $\pm i \boldsymbol{i}$, etc.

By a divided powers signed sequence (dpss for short) we mean a sequence

$$
\boldsymbol{i}=\left(\varepsilon_{1} i_{1}^{\left(a_{1}\right)}, \varepsilon_{2} i_{2}^{\left(a_{2}\right)}, \ldots, \varepsilon_{m} i_{m}^{\left(a_{m}\right)}\right)
$$

where $\varepsilon$ 's and $i$ 's are as before and $a_{1}, \ldots, a_{m} \in\{1,2, \ldots\}$. Let $|\boldsymbol{i}|=\sum_{k=1}^{m} \varepsilon_{k} a_{k} i_{k} \in$ $\mathbb{Z}[I]$ and define $\|\boldsymbol{i}\|$, the length of $\boldsymbol{i}$, as $\sum_{k=1}^{m} a_{k} \in \mathbb{N}$. For a dpss $\boldsymbol{i}$ let $\hat{\boldsymbol{i}}$ be a signed sequence given by expanding $i$

$$
\begin{aligned}
\hat{\boldsymbol{i}} & =\left(\varepsilon_{1} i_{1}, \ldots, \varepsilon_{1} i_{1}, \varepsilon_{2} i_{2}, \ldots, \varepsilon_{2} i_{2}, \ldots, \varepsilon_{m} i_{m}, \ldots, \varepsilon_{m} i_{m}\right) \\
& =\left(\left(\varepsilon_{1} i_{1}\right)^{a_{1}}\left(\varepsilon_{2} i_{2}\right)^{a_{2}} \ldots\left(\varepsilon_{m} i_{m}\right)^{a_{m}}\right),
\end{aligned}
$$

with term $\varepsilon_{1} i_{1}$ repeating $a_{1}$ times, term $\varepsilon_{2} i_{2}$ repeating $a_{2}$ times, etc.

Define

$$
E_{\varepsilon i(a)}:=E_{\varepsilon i}^{(a)}=\frac{E_{\varepsilon i}^{a}}{[a]_{i} !} \in \mathbf{U},
$$

the quantum divided powers of generators $E_{\varepsilon i}$. Then

$$
E_{\varepsilon i(a)} 1_{\lambda}=E_{\varepsilon i}^{(a)} 1_{\lambda} \in \dot{\mathbf{U}}
$$

has left weight $\lambda+\varepsilon a i_{X}$. More generally, for a dpss $\boldsymbol{i}$,

$$
E_{\boldsymbol{i}}:=E_{\varepsilon_{1} i_{1}}^{\left(a_{1}\right)} E_{\varepsilon_{2} i_{2}}^{\left(a_{2}\right)} \ldots E_{\varepsilon_{m} i_{m}}^{\left(a_{m}\right)} \in \mathbf{U}
$$

and

$$
E_{\boldsymbol{i}} 1_{\lambda}:=E_{\varepsilon_{1} i_{1}}^{\left(a_{1}\right)} E_{\varepsilon_{2} i_{2}}^{\left(a_{2}\right)} \ldots E_{\varepsilon_{m} i_{m}}^{\left(a_{m}\right)} 1_{\lambda} \in \dot{\mathbf{U}}
$$

with left weight $\lambda+\sum_{r=1}^{m} \varepsilon_{r} a_{r}\left(i_{r}\right)_{X}$.

Let SSeqd be the set of all dpss. Elements $E_{\boldsymbol{i}} 1_{\lambda}$, over all $\boldsymbol{i} \in \mathrm{SSeqd}$ and $\lambda \in X$, $\operatorname{span}{ }_{A} \dot{\mathbf{U}}$ as a $\mathbb{Z}\left[q, q^{-1}\right]$-module. For $v \in \mathbb{N}[I]$, Seqd(v) was defined in [16] as the set of all expressions $i_{1}^{\left(a_{1}\right)} \ldots i_{m}^{\left(a_{m}\right)}$ such that $\sum_{r=1}^{m} a_{r} i_{r}=v$. Each element of Seqd(v) gives a positive divided power sequence, and

$$
\operatorname{SSeqd}^{+} \cong \coprod_{\nu \in \mathbb{N}[I]} \operatorname{Seqd}(v)
$$

The length $\|\boldsymbol{i}\|$ of a dpss is defined as the sum $a_{1}+a_{2}+\cdots+a_{m}$ in the above notation. 
For the reader's convenience our notations for sequences are collected below:

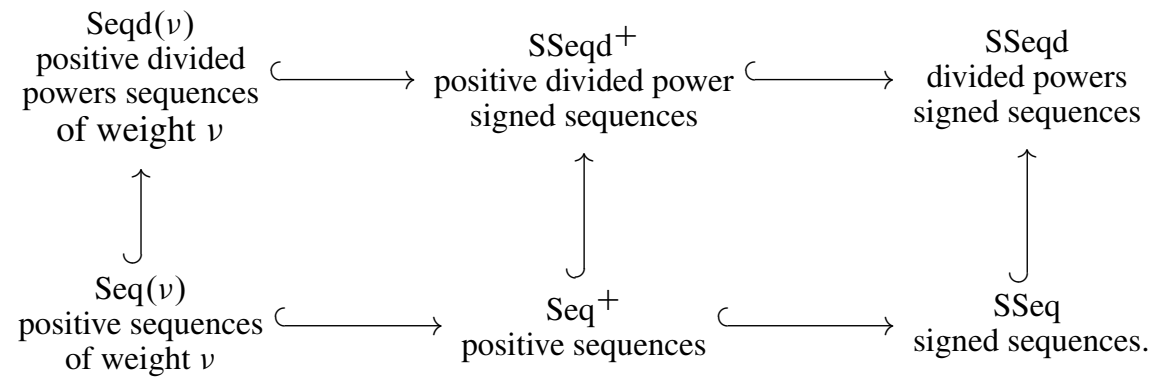

2.2. Geometric interpretation of the bilinear form. Mark $m$ points $1 \times\{0\}, 2 \times\{0\}$, $\ldots, m \times\{0\}$ on the lower boundary $\mathbb{R} \times\{0\}$ of the strip $\mathbb{R} \times[0,1]$ and $k$ points $1 \times\{1\}, 2 \times\{1\}, \ldots, k \times\{1\}$ on the upper boundary $\mathbb{R} \times\{1\}$. Assuming $m+k$ is even, choose an immersion of $\frac{m+k}{2}$ strands into $\mathbb{R} \times[0,1]$ with these $m+k$ points as the endpoints. Orient each strand and label it by an element of $I$. Then endpoints inherit orientations and labels from the strands:
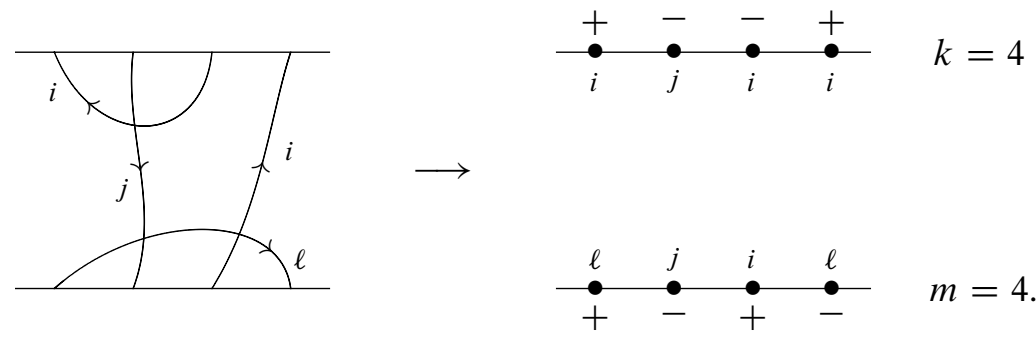

Orientation and labels at lower and upper endpoints define signed sequences $\boldsymbol{i}, \boldsymbol{j} \in$ SSeq. In the above example, $\boldsymbol{i}=(+\ell,-j,+i,-\ell)$ and $\boldsymbol{j}=(+i,-j,-i,+i)$. We consider immersions modulo boundary-preserving homotopies and call them pairings between sequences $\boldsymbol{i}$ and $\boldsymbol{j}$, or simply $(\boldsymbol{i}, \boldsymbol{j})$-pairings.

Clearly, $(\boldsymbol{i}, \boldsymbol{j})$-pairings are in a bijection with complete matchings of $m+k$ points such that the two points in each matching pair share the same label and their orientations are compatible. Denote by $p^{\prime}(\boldsymbol{i}, \boldsymbol{j})$ the set of all $(\boldsymbol{i}, \boldsymbol{j})$-pairings.

A minimal diagram $D$ of a $(\boldsymbol{i}, \boldsymbol{j})$-pairing is a generic immersion that realizes the pairing such that strands have no self-intersections and any two strands intersect at most once. We consider minimal diagrams up to boundary-preserving isotopies. A $(\boldsymbol{i}, \boldsymbol{j})$-pairing has at least one minimal diagram, in the example below it has two.
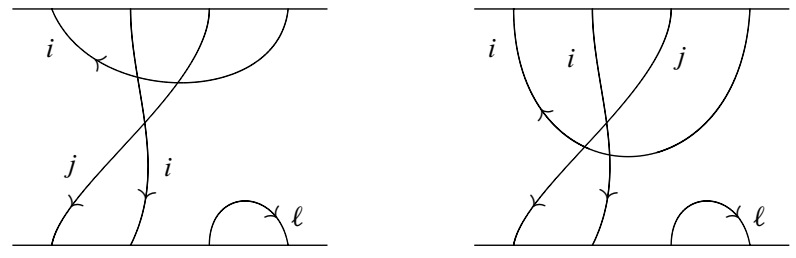
Here is an immersion which is not a minimal diagram.

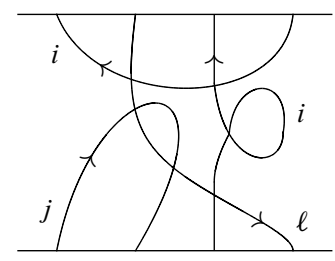

Given $\lambda \in X$, color the regions of a minimal diagram $D$ by elements of $X$ such that the rightmost region is colored $\lambda$, and the two regions between the two sides on an $i$-labelled strand differ by $i_{X}$ :

$$
\lambda+i_{X} \uparrow_{i} \lambda
$$

Isotope $D$ so that the strands at each crossing are either both oriented up or down, and define the degree $\operatorname{deg}(D, \lambda)$ of $D$ relative to $\lambda$ as the integer which is the sum of contributions from all crossings, local maxima and minima of $D$ :

\begin{tabular}{|c|c|c|c|c|c|c|}
\hline deg & $c_{+i, \lambda}$ & $c_{-i, \lambda}$ & $c_{+i, \lambda}$ & $c_{-i, \lambda}$ & $-i \cdot j$ & $-i \cdot j$ \\
\hline
\end{tabular}

where

$$
c_{ \pm i, \lambda}:=\frac{i \cdot i}{2}(1 \pm\langle i, \lambda\rangle)
$$

Notice that

$$
q^{c_{ \pm i, \lambda}}=q_{i}^{1 \pm\langle i, \lambda\rangle} .
$$

In the simply-laced case we have $q_{i}=q$ and $c_{ \pm i, \lambda}=1 \pm\langle i, \lambda\rangle$.

The degrees of the other crossings are determined from the rules above:

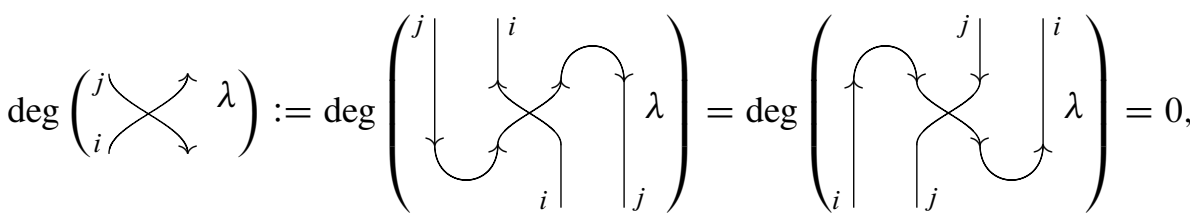

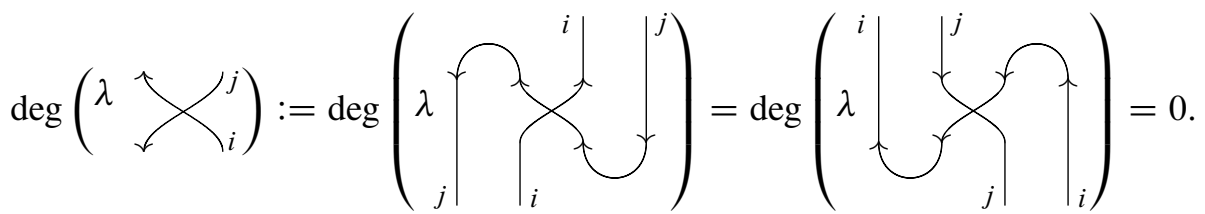


Since both of these crossings have degree zero, we will refer to them as balanced crossings.

Proposition 2.6. $\operatorname{deg}(D, \lambda)$ depends only on $\lambda$ and on the $(\boldsymbol{i}, \boldsymbol{j})$-pairing realized by $D$. Thus, $\operatorname{deg}(D, \lambda)$ is independent of the choice of a minimal diagram for a pairing.

Proof. Invariance under cancellation of U-turns can be shown as follows:

$$
\begin{aligned}
& \operatorname{deg}\left(\uparrow^{\lambda+i_{X}}{ }^{\lambda}\right)=\frac{i \cdot i}{2}(1+\langle i, \lambda\rangle)+\frac{i \cdot i}{2}\left(1-\left\langle i, \lambda+i_{X}\right\rangle\right) \\
& =0=\operatorname{deg}\left(\begin{array}{c|c}
\lambda+i_{X} & \lambda \\
&
\end{array}\right) .
\end{aligned}
$$

Invariance of $\operatorname{deg}(D, \lambda)$ under other isotopies of $D$ is straightforward and is left to the reader. Any two minimal diagrams of the same $(\boldsymbol{i}, \boldsymbol{j})$-pairing are related by a sequence of isotopies and "triple-crossing" moves for various orientations, see examples below.
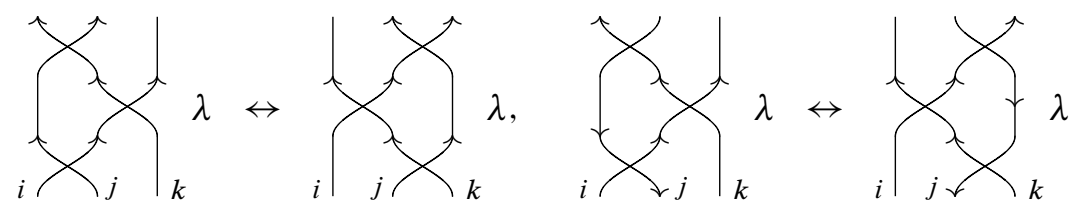

The invariance under these moves is manifestly obvious.

From now on, for each $(\boldsymbol{i}, \boldsymbol{j})$-pairing we choose one minimal diagram $D$ representing this pairing and denote by $p(\boldsymbol{i}, \boldsymbol{j})$ the set of these diagrams. Recall the pairings (, ) and $\langle$,$\rangle on \dot{\mathbf{U}}$ from Section 2.1. The following theorem generalizes similar results from [16], [17].

Theorem 2.7. For any $\boldsymbol{i}, \boldsymbol{j} \in \mathrm{SSeq}$ and $\lambda, \mu \in X$ we have

$$
\left(E_{\boldsymbol{i}} 1_{\lambda}, E_{\boldsymbol{j}} 1_{\mu}\right)=\left\langle E_{\boldsymbol{i}} 1_{\lambda}, E_{\boldsymbol{j}} 1_{\mu}\right\rangle=\delta_{\lambda \mu} \sum_{D \in p(\boldsymbol{i}, \boldsymbol{j})} q^{\operatorname{deg}(D, \lambda)} \prod_{i \in I} \frac{1}{\left(1-q_{i}^{2}\right)^{\kappa_{i}}},
$$

where $\kappa_{i}$ is the number of $i$-colored strands in $D$. 
For example,
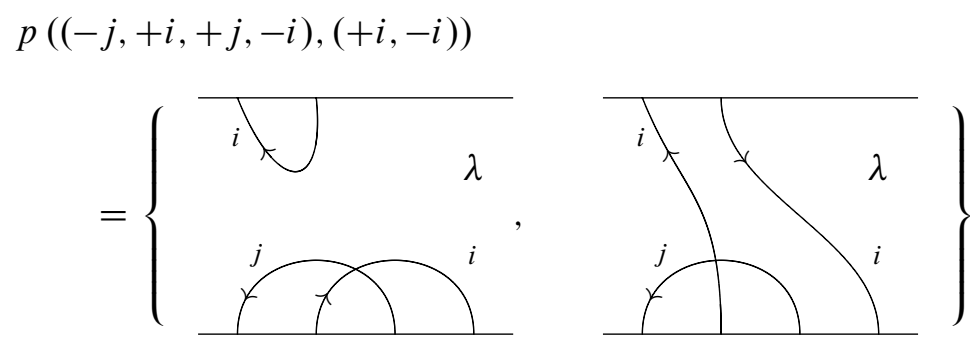

so that

$$
\begin{aligned}
& \left(E_{(-j,+i,+j,-i)} 1_{\lambda}, E_{(+i,-i)} 1_{\lambda}\right) \\
& \quad=\left(q^{2 c_{-i, \lambda}+c_{+j, \lambda}-i \cdot j}+q^{c_{+j, \lambda-i}}\right) \frac{1}{\left(1-q_{i}\right)^{2}} \frac{1}{\left(1-q_{j}\right)} \\
& \quad=\left(q_{i}^{2(1-\langle i, \lambda\rangle)} q_{j}^{(1+\langle j, \lambda\rangle)} q^{-i \cdot j}+q_{j}^{(1+\langle j, \lambda\rangle)} q^{-i \cdot j}\right) \frac{1}{\left(1-q_{i}\right)^{2}} \frac{1}{\left(1-q_{j}\right)} .
\end{aligned}
$$

The product term in (2.2) is independent of $D$ since $2 \kappa_{i}$ is the number of times label $i$ appears in the sequence $\boldsymbol{i} \boldsymbol{j}$.

Proof. The first equality in (2.2) is obvious since $E_{\boldsymbol{i}} 1_{\lambda}$ is invariant under the involution $\psi$. For $\boldsymbol{i}, \boldsymbol{j}, \lambda, \mu$ as in Theorem 2.7 define

$$
\left(E_{\boldsymbol{i}} 1_{\lambda}, E_{\boldsymbol{j}} 1_{\mu}\right)^{\prime}=\delta_{\lambda \mu} \sum_{D \in p(\boldsymbol{i}, \boldsymbol{j})} q^{\operatorname{deg}(D, \lambda)} \prod_{i \in I} \frac{1}{\left(1-q_{i}^{2}\right)^{\kappa_{i}}} .
$$

During the proof we view $E_{\boldsymbol{i}} 1_{\lambda}$ and $E_{\boldsymbol{j}} 1_{\mu}$ in $\left(E_{\boldsymbol{i}} 1_{\lambda}, E_{\boldsymbol{j}} 1_{\mu}\right)^{\prime}$ as formal symbols rather than elements of $\dot{\mathbf{U}}$, since we have not yet proved that (, $)^{\prime}$ descends to $\dot{\mathbf{U}}$. We want to show

$$
\left(E_{\boldsymbol{i}} 1_{\lambda}, E_{\boldsymbol{j}} 1_{\mu}\right)^{\prime}=\left(E_{\boldsymbol{i}} 1_{\lambda}, E_{\boldsymbol{j}} 1_{\mu}\right)
$$

for all $\boldsymbol{i}, \boldsymbol{j}, \lambda$ and $\mu$.

The nontrivial case is $\lambda=\mu$. We can also interpret the formula $\left(E_{\boldsymbol{i}} 1_{\lambda}, E_{\boldsymbol{j}} 1_{\mu}\right)^{\prime}=$ 0 for $\mu \neq \lambda$ via the sum of diagrams, since then the rightmost region must be colored by both $\lambda$ and $\mu$, which is not allowed.

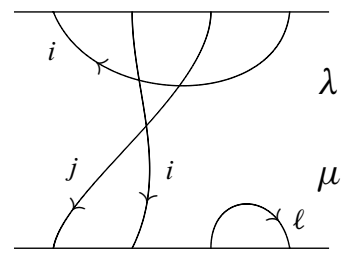

Notice that in the absence of $(\boldsymbol{i}, \boldsymbol{j})$-pairings the right-hand side of (2.2) is zero, since there are no diagrams to sum over. If the left weights of $E_{\boldsymbol{i}} 1_{\lambda}$ and $E_{\boldsymbol{j}} 1_{\lambda}$ are 
different the inner product $\left(E_{\boldsymbol{i}} 1_{\lambda}, E_{\boldsymbol{j}} 1_{\lambda}\right)=0$ and $\left(E_{\boldsymbol{i}} 1_{\lambda}, E_{\boldsymbol{j}} 1_{\lambda}\right)^{\prime}=0$ as well since the set of $(\boldsymbol{i}, \boldsymbol{j})$-pairings is empty in this case.

Assume that both $\boldsymbol{i}$ and $\boldsymbol{j}$ are positive. Then any minimal $(\boldsymbol{i}, \boldsymbol{j})$-diagram can be isotoped to be braid-like, with the strands going upward.
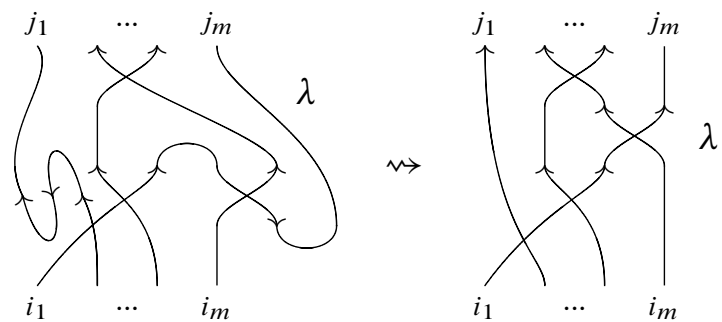

Moreover, in this case $k=m$, otherwise $p(\boldsymbol{i}, \boldsymbol{j})$ is empty. The sum on the righthand side of (2.2) describes the canonical bilinear form on $\mathbf{U}^{+} \cong \mathbf{f}$ evaluated on $E_{\boldsymbol{i}}=E_{i_{1}} \ldots E_{i_{m}}$ and $E_{\boldsymbol{j}}=E_{j_{1}} \ldots E_{j_{m}}$, see [25], [27], [16], [17].

In view of property (iv) of Lusztig's bilinear form, we obtain the following:

Lemma 2.8. If $\boldsymbol{i}, \boldsymbol{j}$ are positive,

$$
\left(E_{\boldsymbol{i}} 1_{\lambda}, E_{\boldsymbol{j}} 1_{\lambda}\right)^{\prime}=\left(E_{\boldsymbol{i}} 1_{\lambda}, E_{\boldsymbol{j}} 1_{\lambda}\right)
$$

for any $\lambda \in X$.

Thus, for positive $\boldsymbol{i}, \boldsymbol{j}$, the geometrically defined bilinear form $(,)^{\prime}$ coincides with $($,$) .$

Lemma 2.9. Equation (2.2) holds for the pair $( \pm i \boldsymbol{i}, \boldsymbol{j})$ if and only if it holds for the $\operatorname{pair}(\boldsymbol{i}, \mp i \boldsymbol{j})$.

Proof. Attaching a U-turn gives a bijection between $p( \pm i \boldsymbol{i}, \boldsymbol{j})$ and $p(\boldsymbol{i}, \mp i \boldsymbol{j})$.
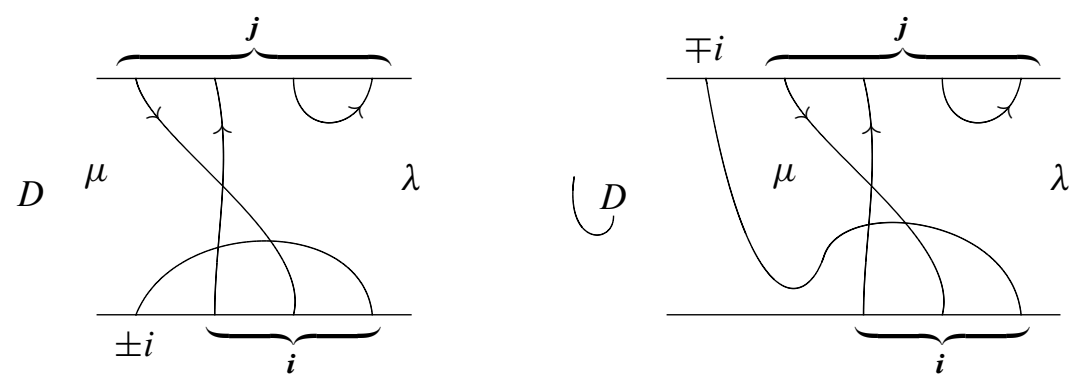

We have

$\operatorname{deg}(\bigcup D, \lambda)=\operatorname{deg}(D, \lambda)+\frac{i \cdot i}{2}\left(1 \pm\left\langle i, \mu \mp i_{X}\right\rangle\right)=\operatorname{deg}(D, \lambda)-\frac{i \cdot i}{2}(1 \mp\langle i, \mu\rangle)$,

where the additional term matches the power of $q$ in the formula $\bar{\rho}\left(1_{\mu} E_{ \pm i}\right)=$ $q_{i}^{1 \mp\langle i, \mu\rangle} E_{\mp i} 1_{\mu}$. 
The previous lemma implies that it is enough to check the equality

$$
\left(E_{\boldsymbol{i}} 1_{\lambda}, E_{\boldsymbol{j}} 1_{\lambda}\right)^{\prime}=\left(E_{\boldsymbol{i}} 1_{\lambda}, E_{\boldsymbol{j}} 1_{\lambda}\right)
$$

when $\boldsymbol{j}$ is the empty sequence. The next three lemmas show that

$$
\left(E_{\boldsymbol{i}} 1_{\lambda}, 1_{\lambda}\right)^{\prime}=\left(E_{\boldsymbol{i}} 1_{\lambda}, 1_{\lambda}\right)
$$

for any $i$ and $\lambda$.

Lemma 2.10. Equation (2.2) holds for all pairs $((-\boldsymbol{j}) \boldsymbol{i}, \emptyset)$ with $\boldsymbol{i}, \boldsymbol{j}$ positive.

Proof. Bending up $-\boldsymbol{j}$ by adding U-turns transforms the pair $((-\boldsymbol{j}) \boldsymbol{i}, \emptyset)$ to $(\boldsymbol{i}, \boldsymbol{j})$ for which (2.2) holds (see Lemma 2.8). We have

$$
\left(E_{(-\boldsymbol{j}) \boldsymbol{i}} 1_{\lambda}, 1_{\lambda}\right)=q^{\alpha}\left(E_{\boldsymbol{i}} 1_{\lambda}, E_{\boldsymbol{j}} 1_{\lambda}\right), \quad\left(E_{(-\boldsymbol{j}) \boldsymbol{i}} 1_{\lambda}, 1_{\lambda}\right)^{\prime}=q^{\alpha}\left(E_{\boldsymbol{i}} 1_{\lambda}, E_{\boldsymbol{j}} 1_{\lambda}\right)^{\prime},
$$

$\alpha$ being the power of $q$ in the formula $\bar{\rho}\left(E_{-\boldsymbol{j}} 1_{\lambda+\boldsymbol{i}_{X}}\right)=\tau\left(E_{-\boldsymbol{j}} 1_{\lambda+\boldsymbol{i}_{X}}\right)=q^{\alpha} 1_{\lambda+\boldsymbol{i}_{X}} E_{\boldsymbol{j}}$.

Lemma 2.11. For $i, j \in I, i \neq j$

$$
\left(E_{\boldsymbol{i}^{\prime} \pm i \mp j \boldsymbol{i}^{\prime \prime}} 1_{\lambda}, 1_{\lambda}\right)^{\prime}=\left(E_{\boldsymbol{i}^{\prime} \pm i \mp j \boldsymbol{i}^{\prime \prime}} 1_{\lambda}, 1_{\lambda}\right)
$$

if and only if

$$
\left(E_{\boldsymbol{i}^{\prime} \mp j \pm i \boldsymbol{i}^{\prime \prime}} 1_{\lambda}, 1_{\lambda}\right)^{\prime}=\left(E_{\boldsymbol{i}^{\prime} \mp j \pm i \boldsymbol{i}^{\prime \prime}} 1_{\lambda}, 1_{\lambda}\right) .
$$

Proof. Attach a crossing at the $\pm i \mp j$ location to a diagram $D$ in $p\left(\boldsymbol{i}^{\prime} \pm i \mp j \boldsymbol{i}^{\prime \prime}, \emptyset\right)$.
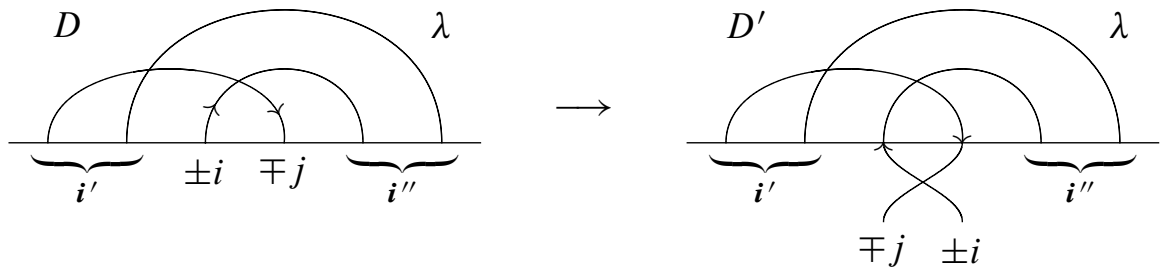

The resulting diagram $D^{\prime}$ is minimal if the $\pm i$ and $\mp j$ strands of $D$ do not intersect. Otherwise, it is not minimal, but the homotopy

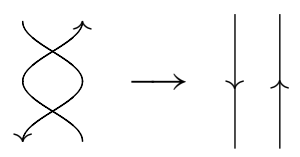

will make it minimal (the orientations in the picture are for the $+i-j$ case).

We get a bijection

$$
p\left(\boldsymbol{i}^{\prime} \pm i \mp j \boldsymbol{i}^{\prime \prime}, \varnothing\right) \stackrel{\cong}{\longrightarrow} p\left(\boldsymbol{i}^{\prime} \mp j \pm i \boldsymbol{i}^{\prime \prime}, \varnothing\right)
$$


which preserves the degree of a diagram, for any $\lambda$, since $\operatorname{deg}(\measuredangle \mu)=0$ for any $\mu$. Hence,

$$
\left(E_{\boldsymbol{i}^{\prime} \pm i \mp j \boldsymbol{i}^{\prime \prime}} 1_{\lambda}, 1_{\lambda}\right)^{\prime}=\left(E_{\boldsymbol{i}^{\prime} \mp j \pm i \boldsymbol{i}^{\prime \prime}} 1_{\lambda}, 1_{\lambda}\right)^{\prime} .
$$

Since $E_{\boldsymbol{i}^{\prime} \pm i \mp j \boldsymbol{i}^{\prime \prime}} 1_{\lambda}=E_{\boldsymbol{i}^{\prime} \mp j \pm i \boldsymbol{i}^{\prime \prime}} 1_{\lambda}$ in $\dot{\mathbf{U}}$, we see that

$$
\left(E_{\boldsymbol{i}^{\prime} \pm i \mp j \boldsymbol{i}^{\prime \prime}} 1_{\lambda}, 1_{\lambda}\right)=\left(E_{\boldsymbol{i}^{\prime} \mp j \pm i \boldsymbol{i}^{\prime \prime}} 1_{\lambda}, 1_{\lambda}\right)
$$

and the lemma follows.

Lemma 2.12. Assume that $\left(E_{\boldsymbol{i}^{\prime} \boldsymbol{i}^{\prime \prime}} 1_{\lambda}, 1_{\lambda}\right)^{\prime}=\left(E_{\boldsymbol{i}^{\prime} \boldsymbol{i}^{\prime \prime}} 1_{\lambda}, 1_{\lambda}\right)$. Then

$$
\left(E_{\boldsymbol{i}^{\prime}+i-i \boldsymbol{i}^{\prime \prime}} 1_{\lambda}, 1_{\lambda}\right)^{\prime}=\left(E_{\boldsymbol{i}^{\prime}+i-i \boldsymbol{i}^{\prime \prime}} 1_{\lambda}, 1_{\lambda}\right)
$$

if and only if

$$
\left(E_{\boldsymbol{i}^{\prime}-i+i \boldsymbol{i}^{\prime \prime}} 1_{\lambda}, 1_{\lambda}\right)^{\prime}=\left(E_{\boldsymbol{i}^{\prime}-i+i \boldsymbol{i}^{\prime \prime}} 1_{\lambda}, 1_{\lambda}\right) .
$$

Proof. Decompose the diagrams in $p\left(\boldsymbol{i}^{\prime}+i-i \boldsymbol{i}^{\prime \prime}, \emptyset\right)$ into three classes:

(1) $+i$ and $-i$ strands do not intersect

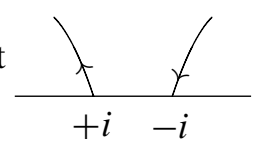

(2) $+i$ and $-i$ strands intersect

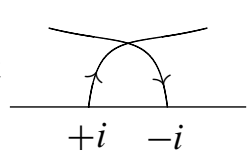

(3) a strand connects $+i$ and $-i$

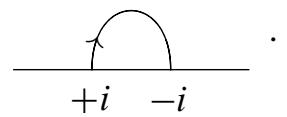

Likewise, decompose the diagrams in $p\left(\boldsymbol{i}^{\prime}-i+i \boldsymbol{i}^{\prime \prime}, \varnothing\right)$ into three classes:

(1) $-i$ and $+i$ strands do not intersect

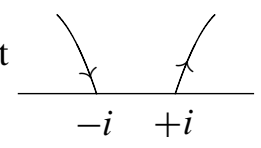

(2) $-i$ and $+i$ strands intersect

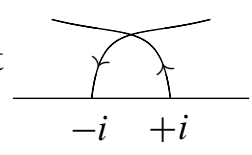

(3) a strand connects $-i$ and $+i$

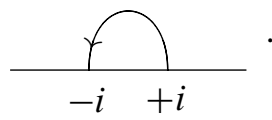


Set up a bijection

$$
p\left(\boldsymbol{i}^{\prime}+i-i \boldsymbol{i}^{\prime \prime}, \emptyset\right) \stackrel{\cong}{\longrightarrow} p\left(\boldsymbol{i}^{\prime}-i+i \boldsymbol{i}^{\prime \prime}, \emptyset\right)
$$

that takes diagrams from class (1) in the first set to diagrams of class (2) in the second set by adding a crossing, diagrams of class (2) to diagrams of class (1) by removing the crossing $\left(>_{+i}\right)$, and diagrams of class (3) to diagrams of class (3) by reversing the orientation of the $+i-i$ strand.

Let $E_{\boldsymbol{i}^{\prime}+i-i \boldsymbol{i}^{\prime \prime}} 1_{\lambda}=E_{\boldsymbol{i}^{\prime}+i-i} 1_{\mu} E_{\boldsymbol{i}^{\prime \prime}} 1_{\lambda}$, that is, $\mu=\lambda+\boldsymbol{i}_{X}^{\prime \prime}$ is the weight of the region to the right of the strand near $-i$.

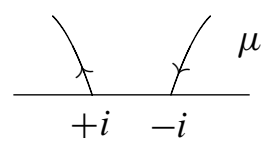

We have in $\dot{\mathbf{U}}$

$$
E_{\boldsymbol{i}^{\prime}+i-i \boldsymbol{i}^{\prime \prime}} 1_{\lambda}=E_{\boldsymbol{i}^{\prime}-i+i \boldsymbol{i}^{\prime \prime}} 1_{\lambda}+[\langle i, \mu\rangle]_{i} E_{\boldsymbol{i}^{\prime} \boldsymbol{i}^{\prime \prime}} 1_{\lambda}
$$

Therefore,

$$
\left(E_{\boldsymbol{i}^{\prime}+i-i \boldsymbol{i}^{\prime \prime}} 1_{\lambda}, 1_{\lambda}\right)=\left(E_{\boldsymbol{i}^{\prime}-i+i \boldsymbol{i}^{\prime \prime}} 1_{\lambda}, 1_{\lambda}\right)+[\langle i, \mu\rangle]_{i}\left(E_{\boldsymbol{i}^{\prime} \boldsymbol{i}^{\prime \prime}} 1_{\lambda}, 1_{\lambda}\right) .
$$

Since $\operatorname{deg}\left(\downarrow^{\prime} \mu\right)=\operatorname{deg}\left(\searrow^{\prime} \mu\right)=0$, a diagram of class (1), respec-

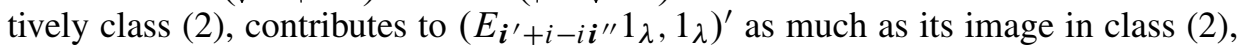
respectively class (1), contributes to $\left(E_{\boldsymbol{i}^{\prime}-i+i \boldsymbol{i}^{\prime \prime}} 1_{\lambda}, 1_{\lambda}\right)^{\prime}$.

A diagrams of class (3) in $p\left(\boldsymbol{i}^{\prime}+i-i \boldsymbol{i}^{\prime \prime}, \emptyset\right)$ comes from some diagram in $p\left(\boldsymbol{i}^{\prime} \boldsymbol{i}^{\prime \prime}, \emptyset\right)$ by adding a $(+i-i)$ cap to the correct position. Similarly, a diagram of class (3) in $p\left(\boldsymbol{i}^{\prime}-i+i \boldsymbol{i}^{\prime \prime}, \emptyset\right)$ comes from a diagram in $p\left(\boldsymbol{i}^{\prime} \boldsymbol{i}^{\prime \prime}, \varnothing\right)$ by adding a $(-i+i)$ cap. Thus, the only contribution to the difference $\left(E_{\boldsymbol{i}^{\prime}+i-i \boldsymbol{i}^{\prime \prime}} 1_{\lambda}, 1_{\lambda}\right)^{\prime}-\left(E_{\boldsymbol{i}^{\prime}-i+i \boldsymbol{i}^{\prime \prime}} 1_{\lambda}, 1_{\lambda}\right)^{\prime}$ comes from class (3) and is given by

$$
\begin{aligned}
& \left(E_{\boldsymbol{i}^{\prime}+i-i \boldsymbol{i}^{\prime \prime}} 1_{\lambda}, 1_{\lambda}\right)^{\prime}-\left(E_{\boldsymbol{i}^{\prime}-i+i \boldsymbol{i}^{\prime \prime}} 1_{\lambda}, 1_{\lambda}\right)^{\prime} \\
& \operatorname{deg}(\overbrace{}^{i}{ }^{\mu}) \operatorname{deg}(\overbrace{\uparrow}^{i}{ }^{\mu}) \\
& =\frac{q}{1-q_{i}^{2}}\left(E_{\boldsymbol{i}^{\prime} \boldsymbol{i}^{\prime \prime}} 1_{\lambda}, 1_{\lambda}\right)^{\prime}-\frac{q}{1-q_{i}^{2}}\left(E_{\boldsymbol{i}^{\prime} \boldsymbol{i}^{\prime \prime}} 1_{\lambda}, 1_{\lambda}\right)^{\prime}
\end{aligned}
$$

or

$$
\begin{aligned}
& \left(E_{\boldsymbol{i}^{\prime}+i-i \boldsymbol{i}^{\prime \prime}} 1_{\lambda}, 1_{\lambda}\right)^{\prime}=\left(E_{\boldsymbol{i}^{\prime}-i+i \boldsymbol{i}^{\prime \prime}} 1_{\lambda}, 1_{\lambda}\right)^{\prime}+\frac{q_{i}^{1-\langle i, \mu\rangle}-q_{i}^{1+\langle i, \mu\rangle}}{1-q_{i}^{2}}\left(E_{\boldsymbol{i}^{\prime} \boldsymbol{i}^{\prime \prime}} 1_{\lambda}, 1_{\lambda}\right)^{\prime}
\end{aligned}
$$

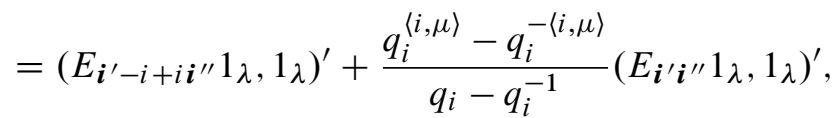


where the coefficient of $\left(E_{\boldsymbol{i}^{\prime} \boldsymbol{i}^{\prime \prime}} 1_{\lambda}, 1_{\lambda}\right)^{\prime}$ in the last term is easily recognized as $[\langle i, \mu\rangle]_{i}$, completing the proof of the lemma.

We can finish the proof that

$$
\left(E_{\boldsymbol{i}} 1_{\lambda}, 1_{\lambda}\right)^{\prime}=\left(E_{\boldsymbol{i}} 1_{\lambda}, 1_{\lambda}\right)
$$

by induction on $\|i\|$, the length of $\boldsymbol{i}$. During the induction step we move all negative entries of $\boldsymbol{i}$ to the left of all positive entries, sometimes adding terms $\left(E_{\boldsymbol{j}} 1_{\lambda}, 1_{\lambda}\right)^{\prime}$, respectively $\left(E_{\boldsymbol{j}} 1_{\lambda}, 1_{\lambda}\right)$ with $\|\boldsymbol{j}\|=\|\boldsymbol{i}\|-2$ to the equation. We can then reduce to the case when all negative entries of $\boldsymbol{i}$ precede all positive entries, which is Lemma 2.10. Theorem 2.7 follows.

We can turn this proof around to define $\dot{\mathbf{U}}$ in a more geometric way than in Lusztig [24]. Start with the $\mathbb{Q}(q)$-algebra' $\mathbf{U}$ with mutually orthogonal idempotents $1_{\lambda}$ and basis $\left\{E_{\boldsymbol{i}} 1_{\lambda}\right\}_{i, \lambda}$, over all finite signed sequences $\boldsymbol{i} \in \mathrm{SSeq}$ and $\lambda \in X$. The multiplication is

$$
E_{\boldsymbol{i}^{\prime}} 1_{\mu} E_{\boldsymbol{i}} 1_{\lambda}= \begin{cases}E_{\boldsymbol{i}^{\prime} \boldsymbol{i}} 1_{\lambda} & \text { if } \mu=\lambda+\boldsymbol{i}_{X}, \\ 0 & \text { otherwise. }\end{cases}
$$

When $\boldsymbol{i}$ is the empty sequence, $E_{\emptyset} 1_{\lambda}=1_{\lambda}$.

Define a $\mathbb{Q}(q)$-bilinear form $(,)^{\prime}$ on $^{\prime} \mathbf{U}$ via the sum over diagrams, formula (2.3). Let $\mathcal{I} \subset{ }^{\prime} \mathbf{U}$ be the kernel of this bilinear form. Then

$$
\mathcal{I}=\bigoplus_{\mu, \lambda \in X}{ }_{\mu} \mathcal{I}_{\lambda}, \quad{ }_{\mu} \mathcal{I}_{\lambda}:=\mathcal{I} \cap{ }_{\mu}{ }^{\mathbf{U}_{\lambda}}
$$

where ${ }_{\mu}{ }^{\prime} \mathbf{U}_{\lambda}$ is spanned by $E_{\boldsymbol{i}} 1_{\lambda}$ for all $\boldsymbol{i}$ such that $\mu=\lambda+\boldsymbol{i}_{X}$. It follows from the definition of the bilinear form that $\mathcal{I}$ is an ideal of ${ }^{\prime} \mathbf{U}$.

It is not hard to check that

$$
\dot{\mathbf{U}} \cong{ }^{\prime} \mathbf{U} / \mathcal{I} \text { and }(,)^{\prime}=(,),
$$

following the above proof of Theorem 2.7. This definition is not too far off from Lusztig's original definition, which utilizes $\mathbf{f} \cong \mathbf{U}^{ \pm}$, defined as the quotient of the free associative algebra ' $\mathbf{f}$ by the kernel of a bilinear form on ' $\mathbf{f}$. The latter bilinear form is the restriction of $(,)^{\prime}$ on ${ }^{\prime} \mathbf{U}$ to ${ }^{\prime} \mathbf{f} \subset^{\prime} \mathbf{U}$. Here we map ' $\mathbf{f} \rightarrow^{\prime} \mathbf{U}$ by sending $\theta_{\boldsymbol{i}}=\theta_{i_{1}} \ldots \theta_{i_{m}}$ to $E_{\boldsymbol{i}} 1_{\lambda}=E_{+i_{1}} \ldots E_{+i_{m}} 1_{\lambda}$ for each positive sequence $\boldsymbol{i}$ (this map is not a homomorphism), alternatively we can send $\theta_{\boldsymbol{i}}$ to $E_{-\boldsymbol{i}} 1_{\lambda}$.

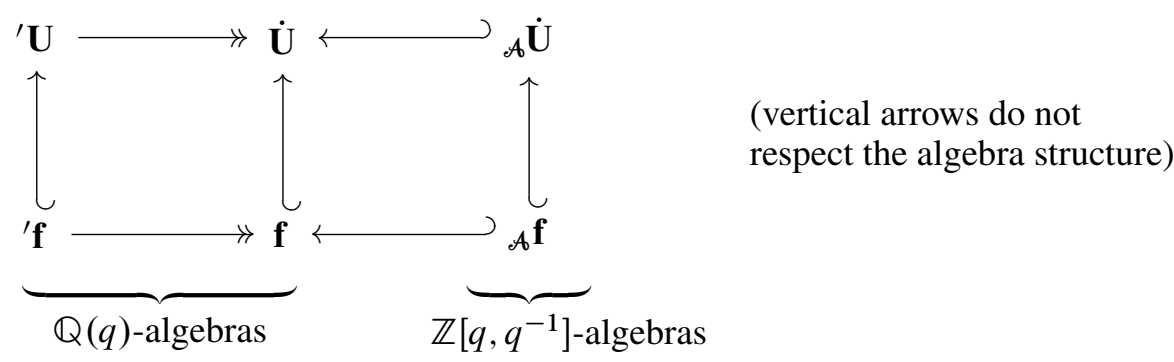


For each $\lambda \in X$ there are two inclusions (corresponding to $\mathbf{U}^{+}$and $\mathbf{U}^{-}$) of the lower half of the diagram to the upper half. Restricting to weight spaces, we get the diagram

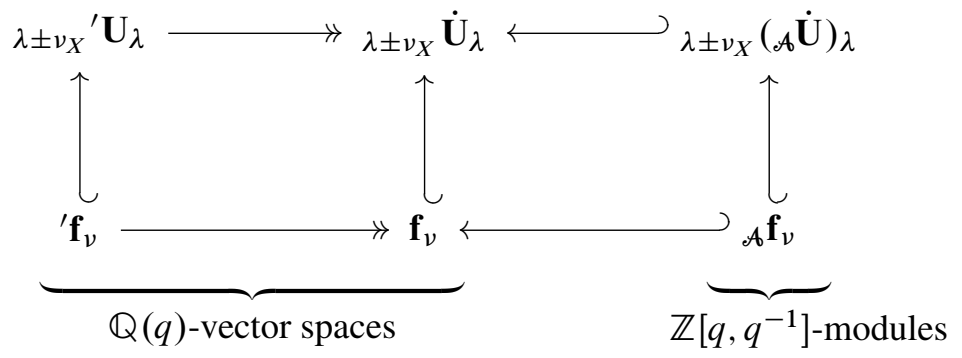

where $\lambda_{ \pm v_{X}} \dot{\mathbf{U}}_{\lambda}=1_{\lambda \pm v_{X}} \dot{\mathbf{U}} 1_{\lambda}$, etc.

\section{Graphical calculus for $\dot{U}$ categorification}

\subsection{The 2-category $u$}

3.1.1. Definition. We define a 2 -category $\mathcal{U}$ for any root datum $(Y, X,\langle\rangle,, \ldots)$ of type $(I, \cdot)$. This 2-category has the structure of an additive $\mathbb{k}$-linear 2-category, see [12] and [21, Section 5]. Thus the hom sets between any two objects form a $\mathbb{k}$ linear category, and composition and identities are given by additive $\mathbb{k}$-linear functors. The 2-morphisms in $\mathcal{U}$ are represented graphically using string diagrams, see [21, Section 4] and the references therein. The categorified $\mathfrak{s l}_{2}$ relations in the definition of $U$ are taken from [21], and the $R(v)$ relations are taken from [16], [17]. A different notion of a categorical $\mathfrak{s l}_{2}$ action appeared earlier in [7].

Definition 3.1. Let $(Y, X,\langle\rangle,, \ldots)$ be a root datum of type $(I, \cdot) \cdot \mathcal{U}$ is an additive $\mathbb{k}$-linear 2-category. The 2-category $U$ consists of

- objects: $\lambda$ for $\lambda \in X$.

The homs $U\left(\lambda, \lambda^{\prime}\right)$ between two objects $\lambda, \lambda^{\prime}$ are additive $\mathbb{k}$-linear categories consisting of the following.

- Objects $^{2}$ of $U\left(\lambda, \lambda^{\prime}\right)$ : a 1-morphism in $\mathcal{U}$ from $\lambda$ to $\lambda^{\prime}$ is a formal finite direct sum of 1-morphisms

$$
\mathcal{E}_{\boldsymbol{i}} \mathbf{1}_{\lambda}\{t\}=\mathbf{1}_{\lambda^{\prime}} \mathcal{E}_{\boldsymbol{i}} \mathbf{1}_{\lambda}\{t\}
$$

for any $t \in \mathbb{Z}$ and signed sequence $i \in$ SSeq such that $\lambda^{\prime}=\lambda+\boldsymbol{i}_{X}$.

- Morphisms of $\mathcal{U}\left(\lambda, \lambda^{\prime}\right)$ : for 1-morphisms $\mathcal{E}_{\boldsymbol{i}} \mathbf{1}_{\lambda}\{t\}, \mathcal{E}_{\boldsymbol{j}} \mathbf{1}_{\lambda}\left\{t^{\prime}\right\} \in \mathcal{U}$, hom sets $U\left(\mathcal{E}_{\boldsymbol{i}} \mathbf{1}_{\lambda}\{t\}, \mathcal{E}_{\boldsymbol{j}} \mathbf{1}_{\lambda}\left\{t^{\prime}\right\}\right)$ of $\mathcal{U}\left(\lambda, \lambda^{\prime}\right)$ are graded $\mathrm{k}$-vector spaces given by linear

\footnotetext{
${ }^{2}$ We refer to objects of the category $\mathcal{U}\left(\lambda, \lambda^{\prime}\right)$ as 1 -morphisms of $\mathcal{U}$. Likewise, the morphisms of $\mathcal{U}\left(\lambda, \lambda^{\prime}\right)$ are called 2 -morphisms in $\mathcal{U}$.
} 
combinations of degree $t-t^{\prime}$ diagrams, modulo certain relations, built from composites of the following.

i) Degree zero identity 2-morphisms $1_{x}$ for each 1-morphism $x$ in $U$. The identity 2-morphisms $1_{\mathcal{E}_{+i} \mathbf{1}_{\lambda}}\{t\}$ and $1_{\mathcal{E}_{-i} \mathbf{1}_{\lambda}}\{t\}$, for $i \in I$, are represented graphically by

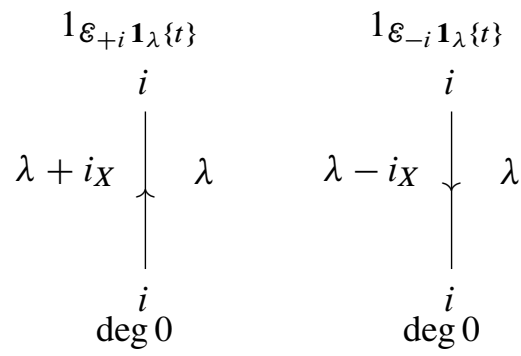

and more generally, for a signed sequence $i=\varepsilon_{1} i_{1} \varepsilon_{2} i_{2} \ldots \varepsilon_{m} i_{m}$, the identity $1_{\mathcal{E}_{\boldsymbol{i}} \mathbf{1}_{\lambda}\{t\}} 2$-morphism is represented as

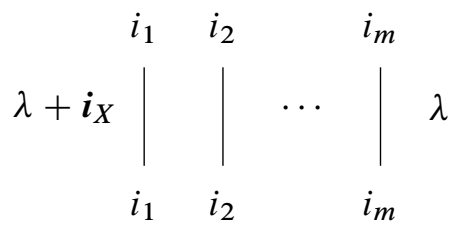

where the strand labelled $i_{\alpha}$ is oriented up if $\varepsilon_{\alpha}=+$ and oriented down if $\varepsilon_{\alpha}=-$. We will often place labels with no sign on the side of a strand and omit the labels at the top and bottom. The signs can be recovered from the orientations on the strands as explained in Section 2.2.

ii) For each $\lambda \in X$ the 2-morphisms

\begin{tabular}{|l|c|c|c|c|}
\hline Notation: & $\hat{\bullet}_{i, \lambda}$ & $\downarrow_{i, \lambda}$ & $\chi_{i, j, \lambda}$ & $\searrow_{i, j, \lambda}$ \\
\hline 2-morphism: & $\lambda+i_{X} \uparrow \lambda$ & $\lambda \downarrow_{\lambda+i_{X}}$ & $\bullet_{i}$ & \\
\hline Degree: & $i \cdot i$ & $i \cdot i$ & $-i \cdot j$ & $-i \cdot j$ \\
\hline
\end{tabular}

\begin{tabular}{|c|c|c|c|c|}
\hline Notation: & $\vartheta_{i, \lambda}$ & $\bigcup_{i, \lambda}$ & $\curvearrowleft_{i, \lambda}$ & $\curvearrowright_{i, \lambda}$ \\
\hline 2-morphism: & tr & & & \\
\hline Degree: & $c_{+i, \lambda}$ & $c_{-i, \lambda}$ & $c_{+i, \lambda}$ & $c_{-i, \lambda}$ \\
\hline
\end{tabular}


with $c_{ \pm i, \lambda}$ defined in (2.1), such that the following identities hold.

- The $\mathfrak{s} \mathfrak{l}_{2}$ relations ${ }^{3}$ (all of the strands are labelled by $i$ ):

a) $\mathbf{1}_{\lambda+i_{X}} \mathcal{E}_{+i} \mathbf{1}_{\lambda}$ and $\mathbf{1}_{\lambda} \mathcal{E}_{-i} \mathbf{1}_{\lambda+i_{X}}$ are biadjoint, up to grading shifts:

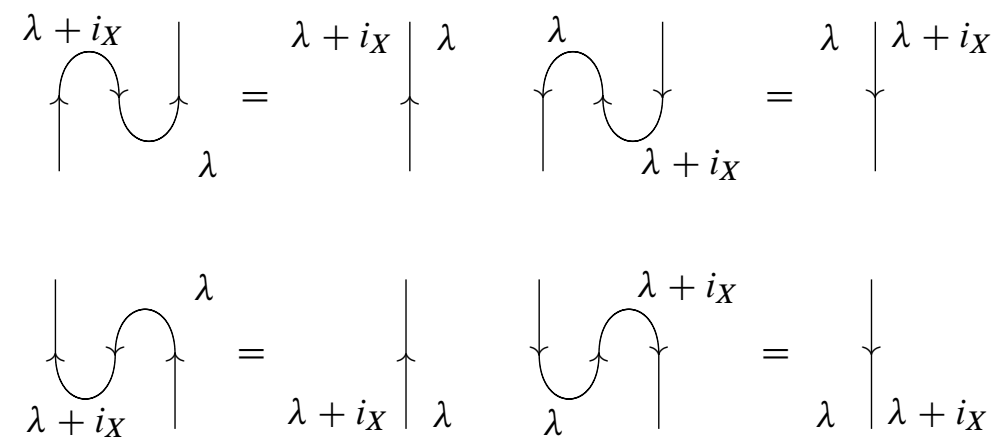

b)

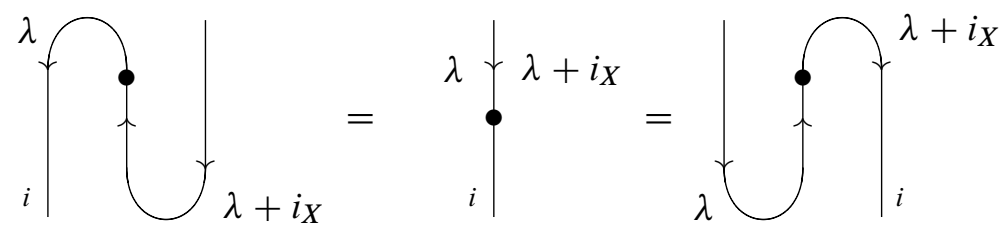

c) All dotted bubbles of negative degree are zero. That is,

$$
\overbrace{\alpha}^{\lambda}=0 \text { if } \alpha<\langle i, \lambda\rangle-1 \overbrace{\alpha}^{\lambda}=0 \text { if } \alpha<-\langle i, \lambda\rangle-1
$$

for all $\alpha \in \mathbb{Z}_{+}$, where a dot carrying a label $\alpha$ denotes the $\alpha$-fold iterated vertical composite of $\hat{\uparrow}_{i, \lambda}$ or $\downarrow_{i, \lambda}$ depending on the orientation. A dotted bubble of degree zero equals 1 :

$$
\overbrace{\langle i, \lambda\rangle-1}^{\lambda}=1 \text { for }\langle i, \lambda\rangle \geq 1 \text {, }
$$$$
\overbrace{-\langle i, \lambda\rangle-1}^{\lambda}=1 \text { for }\langle i, \lambda\rangle \leq-1 \text {. }
$$

d) For the following relations we employ the convention that all summations

\footnotetext{
${ }^{3}$ The vertical $i i$-crossing was represented by the diagram $\oiiint$ in [21], [22]. Here we use a standard crossing for simplicity.
} 
are increasing, so that $\sum_{f=0}^{\alpha}$ is zero if $\alpha<0$.
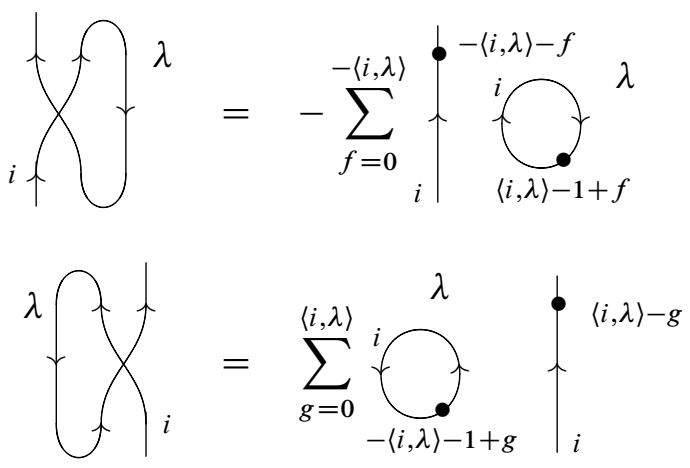

$$
\left.\right|_{\uparrow} \lambda=-\sum_{i}^{\lambda}+\sum_{f=0}^{\langle i, \lambda\rangle-1} \sum_{g=0}^{f}
$$

for all $\lambda \in X$. Notice that for some values of $\lambda$ the dotted bubbles appearing above have negative labels. A composite of $\hat{\bullet}_{i, \lambda}$ or $\mathfrak{\downarrow}_{i, \lambda}$ with itself a negative number of times does not make sense. These dotted bubbles with negative labels, called fake bubbles, are formal symbols inductively defined by the equation

$$
\begin{aligned}
(\overbrace{-\langle i, \lambda\rangle-1}^{\lambda}+\overbrace{-\langle i, \lambda\rangle-1+1}^{\lambda} t+\cdots+ & \overbrace{-\langle i, \lambda\rangle-1+\alpha}^{\lambda} t^{\alpha}+\cdots) \\
& \cdot(\underbrace{i}_{\langle i, \lambda\rangle-1} \hat{\gamma}^{\lambda}+\cdots+\underbrace{\lambda}_{\langle i, \lambda\rangle-1+\alpha} t^{\alpha}+\cdots)
\end{aligned}
$$

and the additional condition

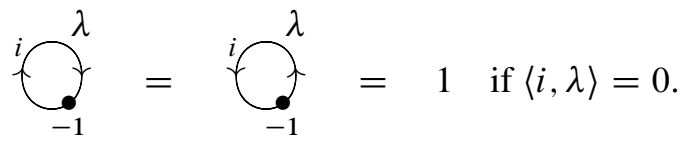

Although the labels are negative for fake bubbles, one can check that the overall degree of each fake bubble is still positive, so that these fake bubbles 
do not violate the positivity of dotted bubble axiom. The above equation, called the infinite Grassmannian relation, remains valid even in high degree when most of the bubbles involved are not fake bubbles. See [21] for more details.

e) NilHecke relations:

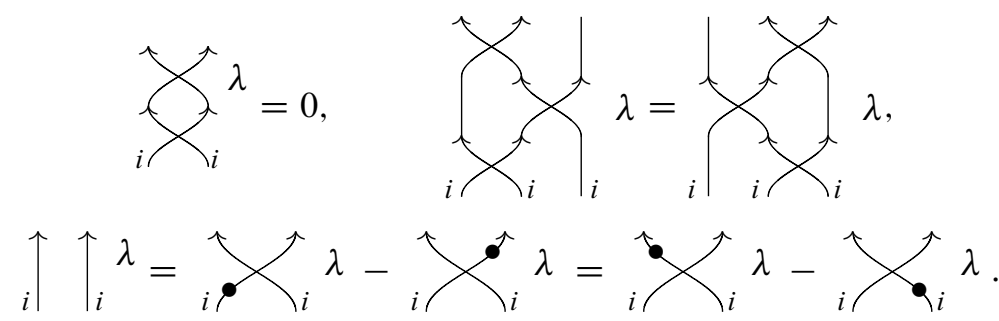

We will also include (3.10) for $i=j$ as an $\mathfrak{s l}_{2}$-relation.

- All 2-morphisms are cyclic ${ }^{4}$ with respect to the above biadjoint structure. This is ensured by the relations (3.3), and the relations

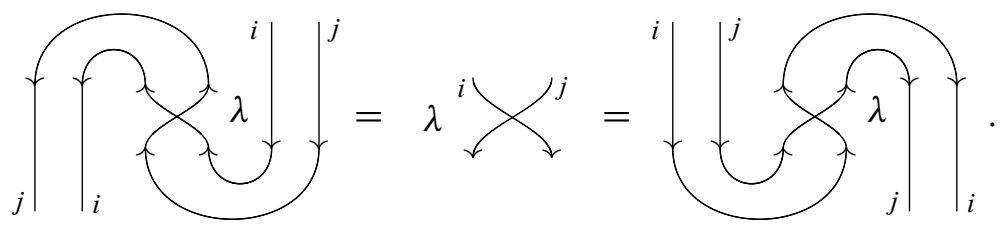

The cyclic condition on 2-morphisms expressed by (3.3) and (3.10) ensures that diagrams related by isotopy represent the same 2-morphism in $\mathcal{U}$. It will be convenient to introduce degree zero 2-morphisms:

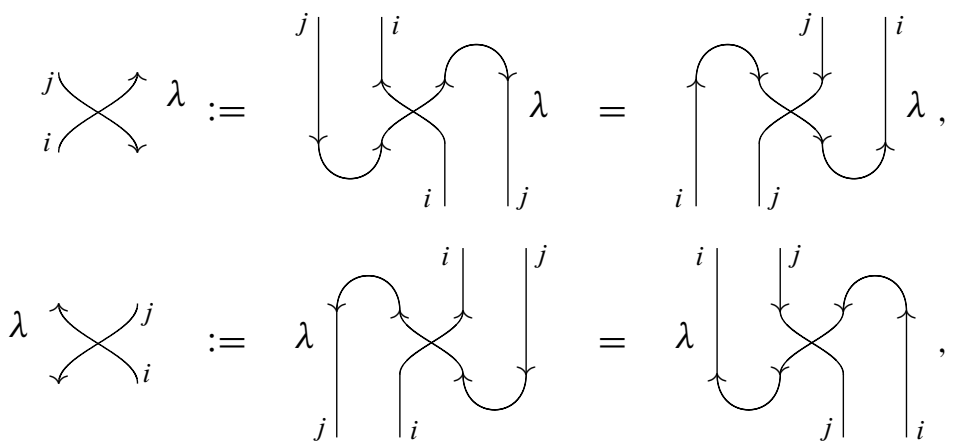

where the second equality in (3.11) and (3.12) follow from (3.10).

\footnotetext{
${ }^{4}$ See [21] and the references therein for the definition of a cyclic 2-morphism with respect to a biadjoint structure.
} 
- For $i \neq j$,

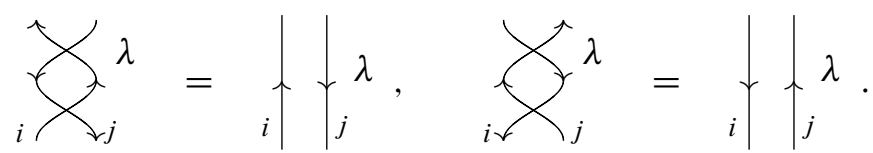

- The $R(v)$-relations:

a) For $i \neq j$,

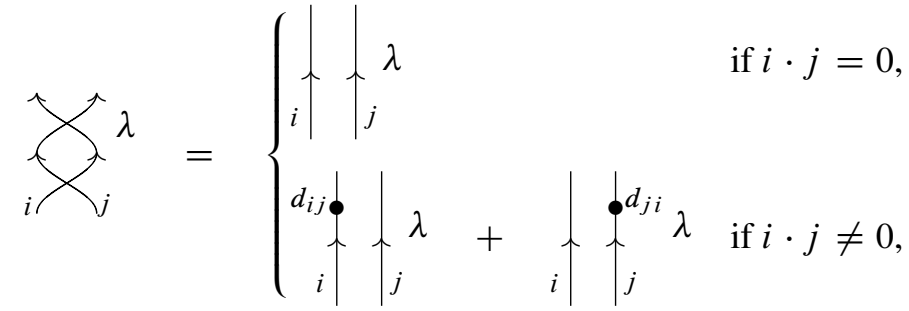

$$
\begin{aligned}
& \uparrow_{i}^{\uparrow} \lambda=\uparrow_{i}^{\lambda} \lambda, \uparrow_{i}^{\lambda} \lambda=\sum_{i}^{\lambda} \lambda .
\end{aligned}
$$

b) Unless $i=k$ and $i \cdot j \neq 0$,

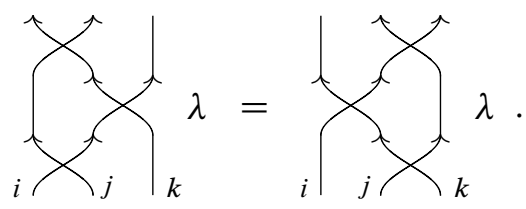

For $i \cdot j \neq 0$,

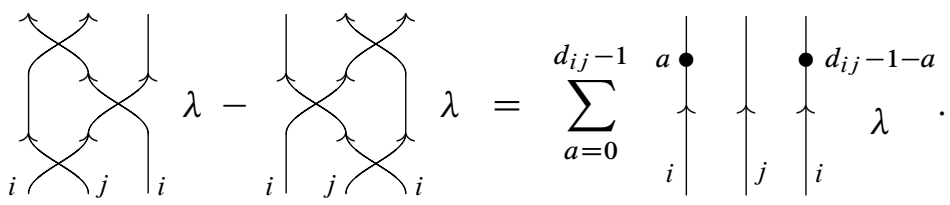

For example, for any shift $t$ there are 2-morphisms

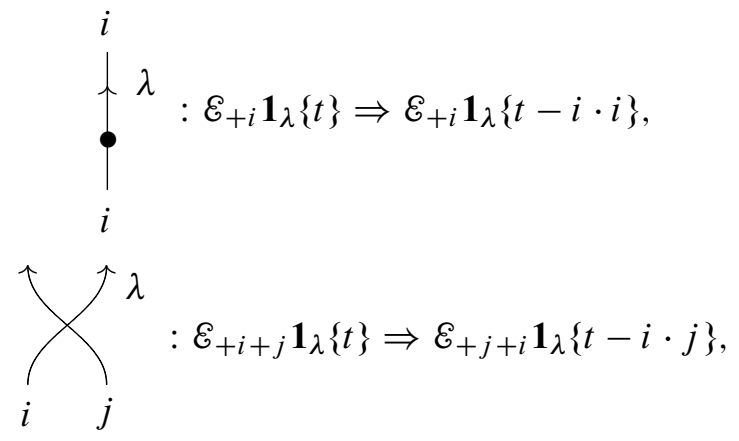




$$
\begin{aligned}
& \succ_{i} \gamma_{\lambda}: \mathbf{1}_{\lambda}\{t\} \Rightarrow \mathcal{E}_{-i+i} \mathbf{1}_{\lambda}\left\{t-c_{+i, \lambda}\right\}, \\
& \gamma^{\lambda}: \varepsilon_{-i+i} \mathbf{1}_{\lambda}\{t\} \Rightarrow \mathbf{1}_{\lambda}\left\{t-c_{-i, \lambda}\right\}
\end{aligned}
$$

in $\mathcal{U}$, and the diagrammatic relation

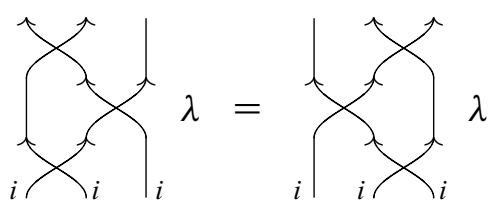

gives rise to relations in $\mathcal{U}\left(\mathcal{E}_{i i i} \mathbf{1}_{\lambda}\{t\}, \mathcal{E}_{i i i} \mathbf{1}_{\lambda}\{t+3 i \cdot i\}\right)$ for all $t \in \mathbb{Z}$.

- The additive $\mathbb{k}$-linear composition functor $\mathcal{U}\left(\lambda, \lambda^{\prime}\right) \times \mathcal{U}\left(\lambda^{\prime}, \lambda^{\prime \prime}\right) \rightarrow \mathcal{U}\left(\lambda, \lambda^{\prime \prime}\right)$ is given on 1-morphisms of $\mathcal{U}$ by

$$
\mathcal{E}_{\boldsymbol{j}} \mathbf{1}_{\lambda^{\prime}}\left\{t^{\prime}\right\} \times \mathcal{E}_{\boldsymbol{i}} \mathbf{1}_{\lambda}\{t\} \mapsto \mathcal{E}_{\boldsymbol{j} \boldsymbol{i}} \mathbf{1}_{\lambda}\left\{t+t^{\prime}\right\}
$$

for $\boldsymbol{i}_{X}=\lambda-\lambda^{\prime}$, and on 2-morphisms of $\mathcal{U}$ by juxtaposition of diagrams.

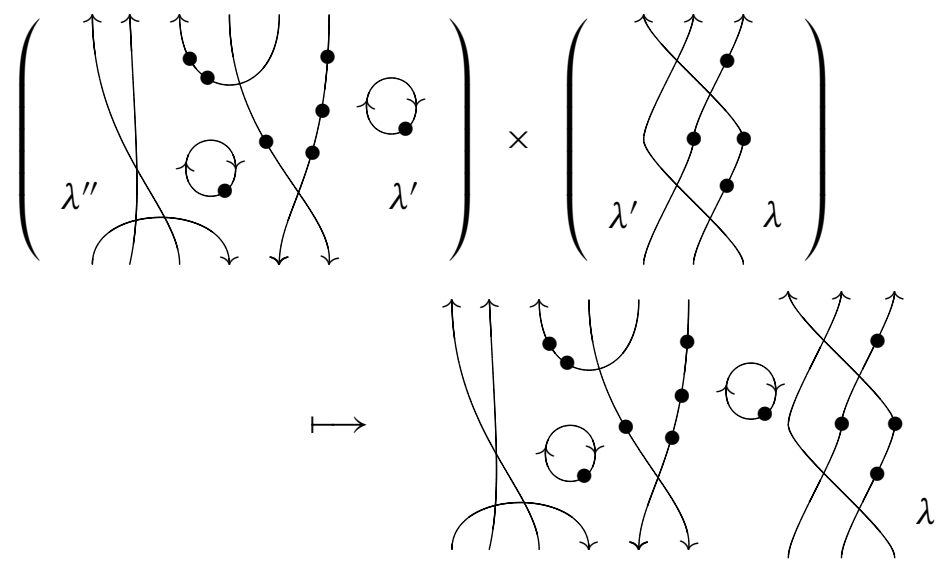

Remark 3.2. By choosing an orientation of the graph associated to Cartan datum $(I, \cdot)$ the $R(v)$-relations above can be modified by replacing them with signed $R(v)$ relations determined by invertible elements $\tau_{i j}, \tau_{j i}$ chosen for each edge of the graph (see [17] for more details).

$\mathcal{U}$ has graded 2-homs defined by

$$
\operatorname{HOM} u(x, y):=\bigoplus_{t \in \mathbb{Z}} \operatorname{Hom} u(x\{t\}, y) .
$$

Also define graded endomorphisms

$$
\operatorname{END}_{u}(x):=\operatorname{HOM}_{u}(x, x) .
$$


The 2-category with the same objects and 1-morphisms as $U$ and 2-homs given by $\operatorname{HOM} u(x, y)$ is denoted $U^{*}$, so that

$$
u^{*}(x, y)=\operatorname{HOM}_{u}(x, y) .
$$

$\mathcal{U}^{*}$ is a graded additive $k$-linear 2-category with translation [21, Section 5.1].

3.1.2. Relations in $\boldsymbol{u}$. We now collect some other relations that follow from those above. These relations simplify computations in the graphical calculus and can be used to reduce complex diagrams into simpler ones (see the proofs of Proposition 3.6 and Lemma 3.9).

Proposition 3.3 (Bubble slides). The following identities hold in $\mathcal{U}$ :

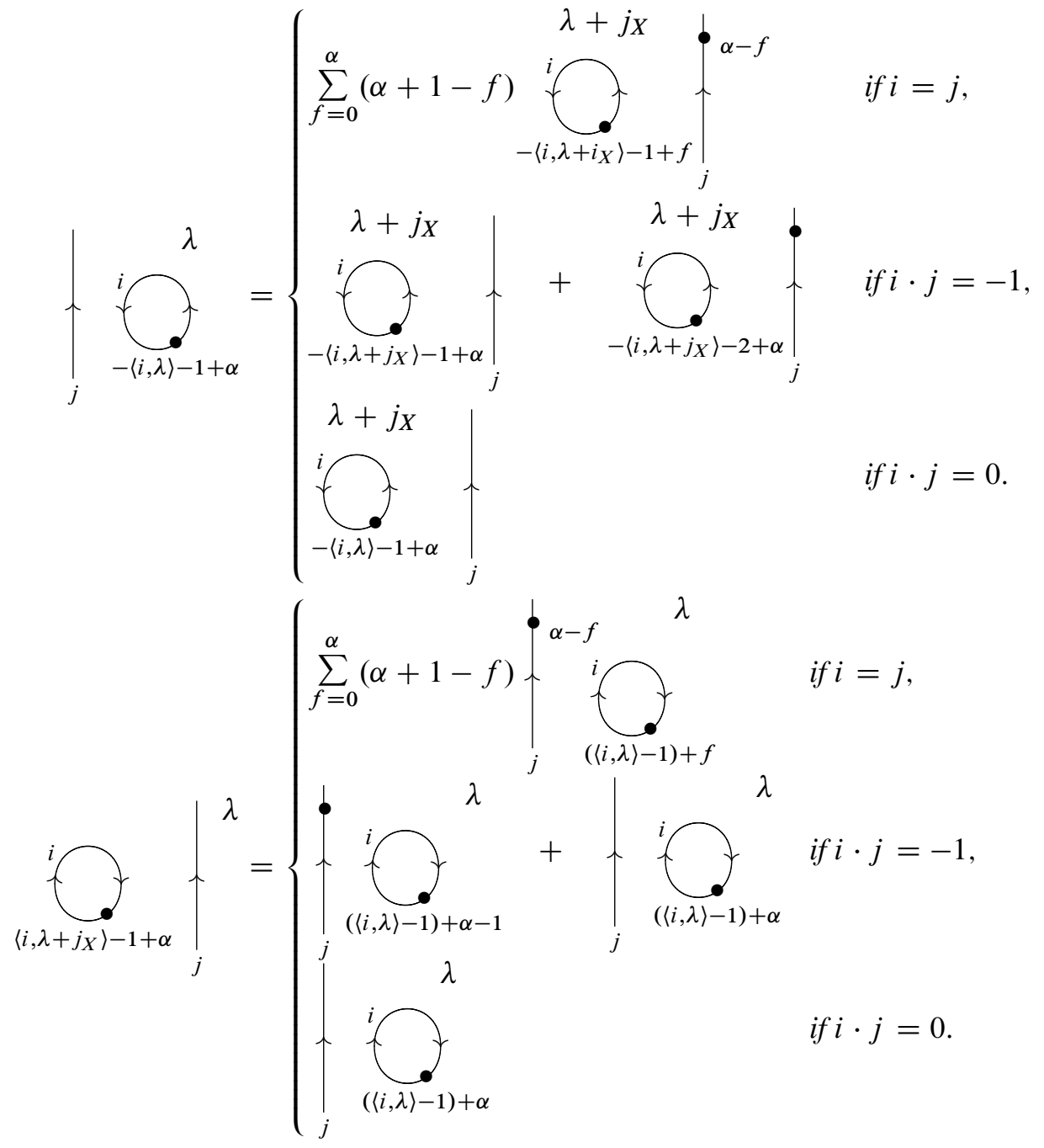


Proof. For $i=j$ the proof appears in [21]. For $i \neq j$ the equation follows from decomposing
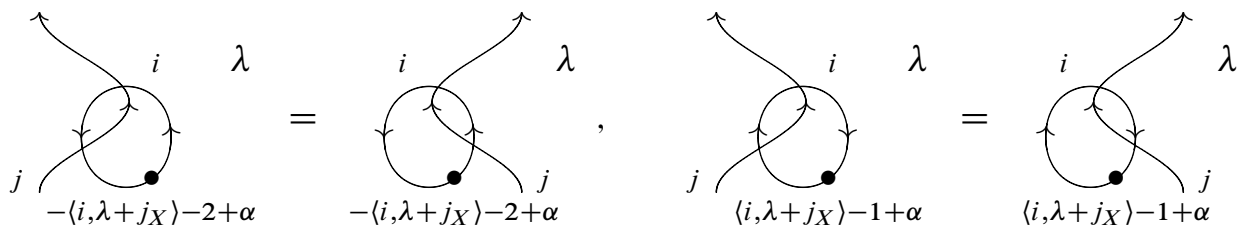

using the relations (3.13) and (3.14).

Proposition 3.4 (More bubble slides). The following identities hold in $\mathcal{U}$ :

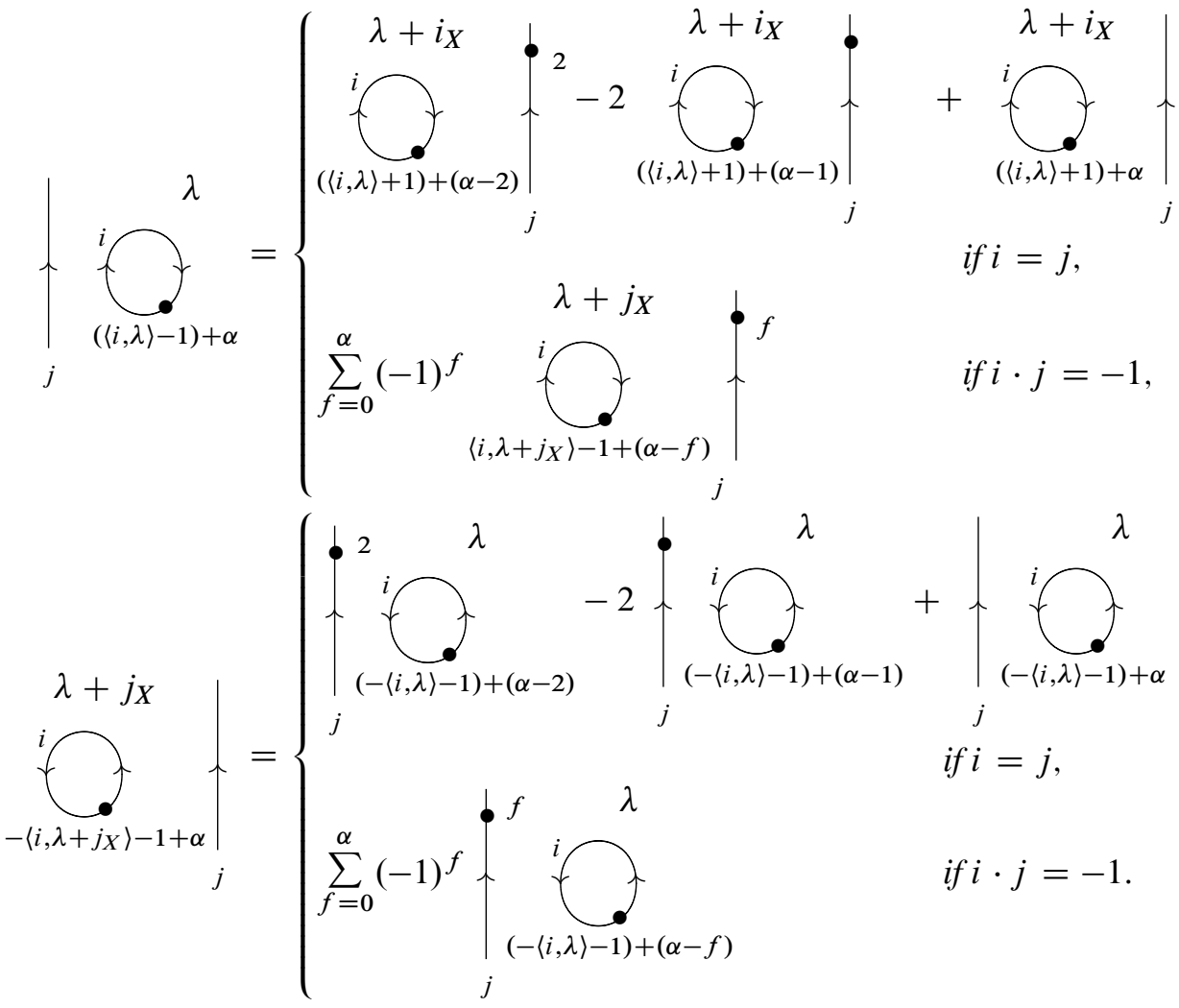

Proof. These equations follow from the previous proposition.

Proposition 3.5. Unless $i=k=j$ we have

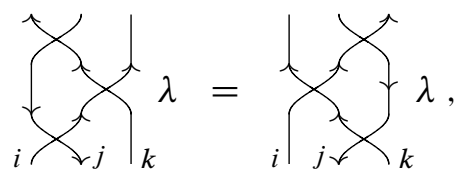


and when $i=j=k$ we have

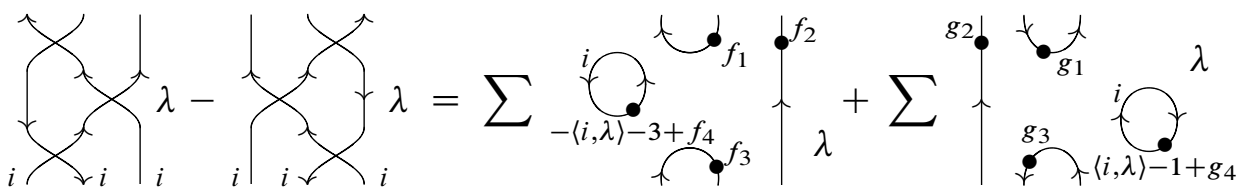

where the first sum is over all $f_{1}, f_{2}, f_{3}, f_{4} \geq 0$ with $f_{1}+f_{2}+f_{3}+f_{4}=\langle i, \lambda\rangle$ and the second sum is over all $g_{1}, g_{2}, g_{3}, g_{4} \geq 0$ with $g_{1}+g_{2}+g_{3}+g_{4}=\langle i, \lambda\rangle-2$. Recall that all summations in this paper are increasing, so that the first summation is zero if $\langle i, \lambda\rangle<0$ and the second is zero when $\langle i, \lambda\rangle<2$.

Reidemeister 3 -like relations for all other orientations are determined from (3.16), (3.17), and the above relations using duality.

Proof. For (3.18) if $i \neq j$ post-compose both sides with the isomorphism

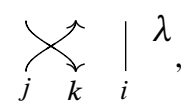

then use that $i=j \neq k$ to apply (3.16) on the right term and establish the equality. If $i=j$ then we may assume that $j \neq k$. In this case we pre-compose with the isomorphism

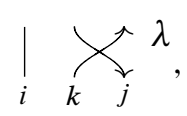

then use (3.16) on the left side to establish the identity.

The case $i=j=k$ appears in [21, Section 5.4].

3.2. Spanning sets of HOMs in $\boldsymbol{u}$. Given two Laurent power series $f(q)=$ $\sum_{k=a}^{+\infty} f_{k} q^{k}$ and $h(q)=\sum_{k=a}^{+\infty}$ with $f_{k}, h_{k} \in \mathbb{Z}$ we say that $f(q) \leq h(q)$ if $f_{k} \leq h_{k}$ for all $k$. For a graded vector space $V=\oplus_{a \in \mathbb{Z}} V_{a}$ define the graded dimension as

$$
\operatorname{gdim} V=\sum_{a \in \mathbb{Z}} q^{a} \operatorname{dim} V_{a}
$$

3.2.1. Endomorphisms of $\mathbf{1}_{\lambda}$. For any root datum and $\lambda \in X$ define a graded commutative ring $\Pi_{\lambda}$ freely generated by symbols

$$
\text { for }\langle i, \lambda\rangle \geq 0 \text { and } \overbrace{\langle i, \lambda\rangle-1+\alpha}^{\lambda} \overbrace{-\langle i, \lambda\rangle-1+\alpha}^{\lambda} \text { for }\langle i, \lambda\rangle<0
$$

of degree $\alpha i \cdot i$ over all $i \in I$ and $\alpha>0$.

Proposition 3.6. Interpreting these generators of $\Pi_{\lambda}$ as elements of $\mathrm{HOM}_{u}\left(\mathbf{1}_{\lambda}, \mathbf{1}_{\lambda}\right)$ induces a surjective graded $\mathbb{k}$-algebra homomorphism

$$
\Pi_{\lambda} \rightarrow \operatorname{HOM}_{u}\left(\mathbf{1}_{\lambda}, \mathbf{1}_{\lambda}\right) .
$$


Proof. By induction on the number of crossings of a closed diagram $D$ representing an endomorphism of $\mathbf{1}_{\lambda}$ one can reduce $D$ to a linear combination of crossingless diagrams following the methods of [21, Section 8]. Crossingless diagrams that contain nested bubbles can be written as linear combinations of crossingless nonnested diagrams using the bubble slide equations in Propositions 3.3 and 3.4. Using the Grassmannian relations (3.7) all dotted bubbles with the same label $i$ can be made to have the same orientation given by (3.19).

The image of the monomial basis of $\Pi_{\lambda}$ under surjective homomorphism (3.20) is a homogeneous spanning set of the graded vector space $\mathrm{HOM}_{U}\left(\mathbf{1}_{\lambda}, \mathbf{1}_{\lambda}\right)$. Denote this spanning set by $B_{\emptyset, \emptyset, \lambda}$.

Let

$$
\pi=\prod_{i \in I} \prod_{a=1}^{\infty} \frac{1}{1-q_{i}^{2 a}} .
$$

$\pi$ depends only on the root datum (on the values of $i \cdot i$ over all $i \in I$ ). The graded dimension of $\Pi_{\lambda}$ is $\pi$.

Corollary 3.7. gdim $\operatorname{HOM} u\left(\mathbf{1}_{\lambda}, \mathbf{1}_{\lambda}\right) \leq \pi$ and $\operatorname{HOM} u\left(\mathbf{1}_{\lambda}, \mathbf{1}_{\lambda}\right)$ is a local graded ring.

Remark 3.8. $\Pi_{\lambda}$ is not Noetherian.

We call monomials in this basis of $\Pi_{\lambda}$ and their images in $\operatorname{HOM}_{U}\left(\mathbf{1}_{\lambda}, \mathbf{1}_{\lambda}\right)$ bubble monomials.

3.2.2. Homs between $\boldsymbol{E}_{\boldsymbol{i}} \mathbf{1}_{\boldsymbol{\lambda}}$ and $\boldsymbol{E}_{\boldsymbol{j}} \mathbf{1}_{\boldsymbol{\lambda}}$ for positive $\boldsymbol{i}$ and $\boldsymbol{j}$. Recall the rings $R(v)$, for $v \in \mathbb{N}[I]$, from [16], [17] defined by unoriented dotted braid-like diagrams $D$ modulo local relations that can be read off from equations (3.8)-(3.9) and (3.14)(3.17) by forgetting the orientation. There is a decomposition

$$
R(v)=\bigoplus_{\boldsymbol{i}, \boldsymbol{j} \in \operatorname{Seq}(v)} j_{j} R(v)_{\boldsymbol{i}},
$$

where ${ }_{j} R(v)_{i}$ is spanned by diagrams $D$ with $\boldsymbol{i}, \boldsymbol{j}$ being the lower and upper sequences of $D$. Adding upward orientations to a diagram $D$ in $R(v)$, placing it to the left of a collection of bubbles representing a monomial in $\Pi_{\lambda}$,
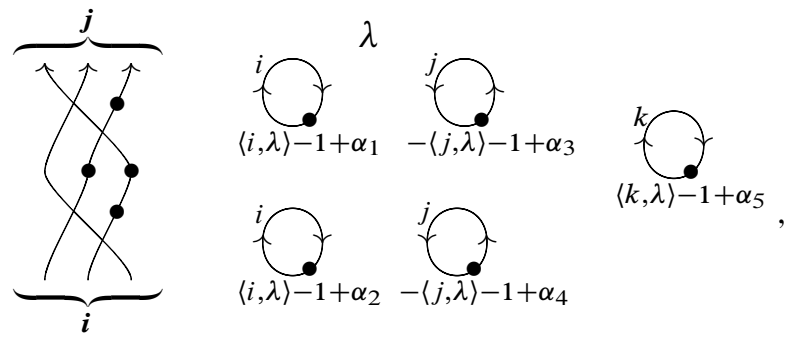
and viewing the result as a 2 -morphism from $\varepsilon_{i} \mathbf{1}_{\lambda}$ to $\mathcal{E}_{\boldsymbol{j}} \mathbf{1}_{\lambda}$ induces a gradingpreserving $\mathbb{k}$-linear map

$$
\varphi_{\boldsymbol{i}, \boldsymbol{j}, \lambda}:{ }_{j} R(\nu)_{\boldsymbol{i}} \otimes_{\mathbb{k}} \Pi_{\lambda} \longrightarrow \operatorname{HOM}_{u}\left(\mathcal{E}_{\boldsymbol{i}} \mathbf{1}_{\lambda}, \mathcal{E}_{\boldsymbol{j}} \mathbf{1}_{\lambda}\right) .
$$

Lemma 3.9. $\varphi_{\boldsymbol{i}, \boldsymbol{j}, \lambda}$ is surjective.

Proof. Start with a diagram $D$ that represents an element in $\operatorname{HOM} u\left(\varepsilon_{\boldsymbol{i}} \mathbf{1}_{\lambda}, \mathcal{E}_{\boldsymbol{j}} \mathbf{1}_{\lambda}\right)$. The relations in our graphical calculus allow us to inductively simplify $D$ by reducing the number of crossings if a strand or a circle of $D$ has a self-intersection or if $D$ contains two strands that intersect more than once. Bubble sliding rules allow moving bubbles to the far right of the diagram. Eventually, $D$ reduces to a linear combination of diagrams which are products of diagrams representing elements of $j R(v)_{i}$ and monomials in $\Pi_{\lambda}$.

The elements in $\operatorname{HOM} u\left(\mathcal{E}_{\boldsymbol{i}} \mathbf{1}_{\lambda}, \mathcal{E}_{\boldsymbol{j}} \mathbf{1}_{\lambda}\right)$ given by diagrams without circles, with no two strands intersecting more than once, and with all dots at the bottom are precisely the image under $\varphi_{\boldsymbol{i}, \boldsymbol{j}, \lambda}$ of the basis $\boldsymbol{j} B_{\boldsymbol{i}}$ of $\boldsymbol{j}_{\boldsymbol{j}} R(v)_{\boldsymbol{i}}$ described in [16]. Denote by $B_{\boldsymbol{i}, \boldsymbol{j}, \lambda}$ the image under $\varphi_{\boldsymbol{i}, \boldsymbol{j}, \lambda}$ of the product basis ${ }_{\boldsymbol{j}} B_{\boldsymbol{i}} \times\left\{\right.$ monomials in $\left.\Pi_{\lambda}\right\}$ in $_{\boldsymbol{j}} R(\nu)_{\boldsymbol{i}} \times \Pi_{\lambda}$. The lemma implies that $B_{\boldsymbol{i}, \boldsymbol{j}, \lambda}$ is a spanning set in $\operatorname{HOM}_{u}\left(\mathcal{E}_{\boldsymbol{i}} \mathbf{1}_{\lambda}, \mathcal{E}_{\boldsymbol{j}} \mathbf{1}_{\lambda}\right)$.

Let

$$
\mathcal{E}_{\nu} \mathbf{1}_{\lambda}:=\bigoplus_{i \in \operatorname{Seq}(v)} \varepsilon_{i} \mathbf{1}_{\lambda}, \quad \varepsilon_{-\nu} \mathbf{1}_{\lambda}:=\bigoplus_{i \in \operatorname{Seq}(v)} \varepsilon_{-i} \mathbf{1}_{\lambda}
$$

$\mathcal{E}_{\nu} \mathbf{1}_{\lambda}$ and $\mathcal{E}_{-\nu} \mathbf{1}_{\lambda}$ are 1-morphisms in $\mathcal{U}$. Summing $\varphi_{\boldsymbol{i}, \boldsymbol{j}, \lambda}$ over all $\boldsymbol{i}, \boldsymbol{j} \in \operatorname{Seq}(\nu)$, we obtain a homomorphism

$$
\varphi_{v, \lambda}: R(v) \otimes_{\mathbb{k}} \Pi_{\lambda} \longrightarrow \operatorname{END} u\left(\varepsilon_{v} \mathbf{1}_{\lambda}\right)=\operatorname{End} u^{*}\left(\varepsilon_{v} \mathbf{1}_{\lambda}\right) .
$$

Proposition 3.10. The homomorphism $\varphi_{v, \lambda}$ of graded $\mathbb{k}$-algebras is surjective.

Adding a downward orientation to a diagram $D$ in $R(v)$, multiplying it by $(-1)^{a}$ where $a$ is the number of crossings of identically colored lines, and placing it to the left of a collection of bubbles representing a monomial in $\Pi_{\lambda}$ induces a surjective homomorphism

$$
\varphi_{-v, \lambda}: R(v) \otimes_{\mathbb{k}} \Pi_{\lambda} \longrightarrow \operatorname{END}_{u}\left(\mathcal{E}_{-\nu} \mathbf{1}_{\lambda}\right) .
$$

3.2.3. Spanning sets for general $\boldsymbol{i}$ and $\boldsymbol{j}$. We now describe a spanning set $B_{\boldsymbol{i}, \boldsymbol{j}, \lambda}$ in $\operatorname{HOM}_{u}\left(\mathcal{E}_{\boldsymbol{i}} \mathbf{1}_{\lambda}, \mathcal{E}_{\boldsymbol{j}} \mathbf{1}_{\lambda}\right)$ for any $\boldsymbol{i}, \boldsymbol{j}, \lambda$. Recall that $p^{\prime}(\boldsymbol{i}, \boldsymbol{j})$ denotes the set of $(\boldsymbol{i}, \boldsymbol{j})$-pairings, and $p(\boldsymbol{i}, \boldsymbol{j})$ is a set of minimal diagrammatic representatives of these pairings. For each diagram $D \in p(\boldsymbol{i}, \boldsymbol{j})$ choose an interval on each of the arcs, away from the intersections. The basis $B_{\boldsymbol{i}, \boldsymbol{j}, \lambda}$ consists of the union, over all $D$, of diagrams built out of $D$ by putting an arbitrary number of dots on each of the intervals and placing any diagram representing a monomial in $\Pi_{\lambda}$ to the right of $D$ decorated by these dots. Notice that $B_{\boldsymbol{i}, \boldsymbol{j}, \lambda}$ depends on extra choices, which we assume are 
made once and for all. For example, for $\boldsymbol{i}=(-j,+i,+j,-i)$ and $\boldsymbol{j}=(+i,-i)$ the choice of minimal diagrams is unique, see example following Theorem 2.7. The choice of intervals for the dots is not unique, though. Choosing these intervals as shown below
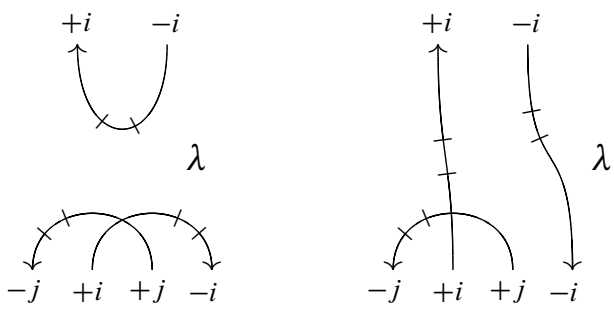

results in the spanning set $B_{(-j,+i,+j,-i),(+i,-i), \lambda}$ whose elements are
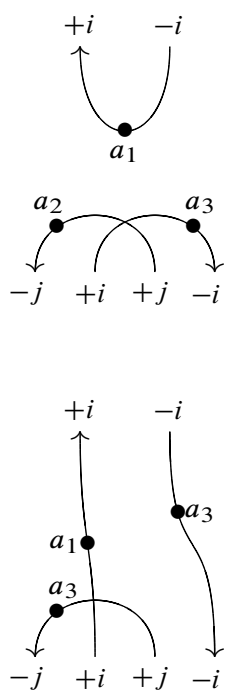

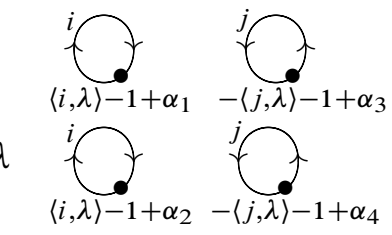

bubble monomial in $\Pi_{\lambda}$
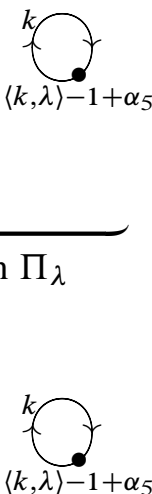

over all nonnegative integers $a_{1}, a_{2}, a_{3}$ and over all diagrammatic monomials in $\Pi_{\lambda}$ (bubble orientations are for the case $\langle i, \lambda\rangle \geq 0,\langle j, \lambda\rangle<0,\langle k, \lambda\rangle \geq 0$ ).

Proposition 3.11. For any intermediate choices made, the set $B_{\boldsymbol{i}, \boldsymbol{j}, \lambda}$ spans the $\mathbb{k}$ vector space $\mathrm{HOM}_{u}\left(\varepsilon_{\boldsymbol{i}} \mathbf{1}_{\lambda}, \mathcal{E}_{\boldsymbol{j}} \mathbf{1}_{\lambda}\right)$.

Proof. Relations on 2-morphisms in $U\left(\varepsilon_{\nu} \mathbf{1}_{\lambda}\right)$ allow arbitrary homotopies of colored dotted diagrams modulo lower order terms, i.e., terms with fewer crossings, fewer circles, etc. Detailed discussion in [21, Section 8] generalizes to the present situation without difficulty.

For each $s \in B_{\boldsymbol{i}, \boldsymbol{j}, \lambda}$ its degree $\operatorname{deg}(s)$ is an integer, determined by the rules in Section 3.1 . 
Proposition 3.12. For $\pi$ as in (3.21) and any $\boldsymbol{i}, \boldsymbol{j}$, and $\lambda$ we have

$$
\pi\left(E_{\boldsymbol{i}} 1_{\lambda}, E_{\boldsymbol{j}} 1_{\lambda}\right)=\sum_{s \in B_{\boldsymbol{i}, \boldsymbol{j}, \lambda}} q^{\operatorname{deg}(s)} .
$$

Proof. The left-hand side of equation (3.23) equals $\pi$ times the RHS of the formula (2.2). This $\pi$ is matched in the right-hand side of (3.23) by the summation over all monomials in $\Pi_{\lambda}$, since $q$ to the degree of these monomials add up to $\pi$. The product term in the right-hand side of (2.2) is matched by the contribution to the right-hand side of (3.23) by all possible placements of dots. For each $i$-labelled strand dots contribute

$$
\sum_{a=0}^{\infty} q^{a(i \cdot i)}=\frac{1}{1-q^{i \cdot i}}=\frac{1}{1-q_{i}^{2}}
$$

to the product, since the degree of a dot is $i \cdot i$, and the sum is over all ways to put some number $a$ of dots on this strand. Finally, the sums over all minimal diagrams $D \in p(\boldsymbol{i}, \boldsymbol{j})$ give equal contributions to the two sides of (3.23).

Remark 3.13. In view of the first equality in (2.2), we can restate the above proposition via $\langle$,$\rangle in place of ($,$) on the left-hand side.$

Notice that

$$
\operatorname{gdim}_{\mathbb{k}}\left(\operatorname{HOM}_{u}\left(\varepsilon_{\boldsymbol{i}} \mathbf{1}_{\lambda}, \mathcal{E}_{\boldsymbol{j}} \mathbf{1}_{\lambda}\right)\right)=\sum_{s \in B_{\boldsymbol{i}, \boldsymbol{j}, \lambda}} q^{\operatorname{deg}(s)}
$$

if and only if $B_{\boldsymbol{i}, \boldsymbol{j}, \lambda}$ is a basis of $\mathrm{HOM}_{u}\left(\mathcal{E}_{\boldsymbol{i}} \mathbf{1}_{\lambda}, \mathcal{E}_{\boldsymbol{j}} \mathbf{1}_{\lambda}\right)$.

Corollary 3.14. For any sequences $\boldsymbol{i}, \boldsymbol{j}$ and $\lambda \in X$ we have

$$
\operatorname{gdim}_{\mathbb{k}}\left(\operatorname{HOM}_{u}\left(\varepsilon_{\boldsymbol{i}} \mathbf{1}_{\lambda}, \mathcal{E}_{\boldsymbol{j}} \mathbf{1}_{\lambda}\right)\right) \leq \pi\left\langle E_{\boldsymbol{i}} 1_{\lambda}, E_{\boldsymbol{j}} 1_{\lambda}\right\rangle .
$$

Definition 3.15. We say that our graphical calculus is non-degenerate for a given root system and field $\mathbb{k}$ if for all $\boldsymbol{i}, \boldsymbol{j}, \lambda$ the set $B_{\boldsymbol{i}, \boldsymbol{j}, \lambda}$ is a basis of $\operatorname{HOM}_{u}\left(\varepsilon_{\boldsymbol{i}} \mathbf{1}_{\lambda}, \mathcal{E}_{\boldsymbol{j}} \mathbf{1}_{\lambda}\right)$.

Thus, a calculus is non-degenerate if the equality holds in Corollary 3.14 for all $\boldsymbol{i}, \boldsymbol{j}, \lambda$.

Remark 3.16. Nondegeneracy holds if the above condition is true for all $\lambda \in X$ and all pairs of positive sequences $\boldsymbol{i}, \boldsymbol{j}$.

Simple non-degeneracy observations. We can assume that $k$ is only a commutative ring, work over this ring from the start, and say that the calculus is non-degenerate over $\mathbb{k}$ if $U^{*}\left(\varepsilon_{\boldsymbol{i}} \mathbf{1}_{\lambda}, \mathcal{E}_{\boldsymbol{j}} \mathbf{1}_{\lambda}\right)$ is a free $\mathrm{k}$-module with a basis $B_{\boldsymbol{i}, \boldsymbol{j}, \lambda}$ for all $\boldsymbol{j}, \boldsymbol{i}$ and $\lambda$. We do not know any examples of a root datum and ring $\mathbb{k}$ when the calculus is degenerate. Over a field the non-degeneracy of the calculus depends only on the root datum and the characteristic of $\mathbb{k}$. If the calculus is non-degenerate over $\mathbb{Q}$, it is non-degenerate over $\mathbb{Z}$ and over any commutative ring $\mathbb{k}$. 
3.2.4. Endomorphisms of $\boldsymbol{E}_{\boldsymbol{v},-\boldsymbol{v}^{\prime}} \mathbf{1}_{\boldsymbol{\lambda}}$. For $v, v^{\prime} \in \mathbb{N}[I]$ let

$$
\mathcal{E}_{v,-v^{\prime}} \mathbf{1}_{\lambda}:=\bigoplus_{\substack{\boldsymbol{i} \in \operatorname{Seq}(v) \\ \boldsymbol{j} \in \operatorname{Seq}\left(v^{\prime}\right)}} \mathcal{E}_{\boldsymbol{i}-\boldsymbol{j}} \mathbf{1}_{\lambda} .
$$

Consider the graded ring $\operatorname{END} u\left(\varepsilon_{v,-v^{\prime}} \mathbf{1}_{\lambda}\right)$. A spanning set for this ring is given by dotted minimal diagrams of $\left(\boldsymbol{i}\left(-\boldsymbol{i}^{\prime}\right), \boldsymbol{j}\left(-\boldsymbol{j}^{\prime}\right)\right)$-pairings over all $\boldsymbol{i}, \boldsymbol{j} \in \operatorname{Seq}(v)$, $\boldsymbol{i}^{\prime}, \boldsymbol{j}^{\prime} \in \operatorname{Seq}\left(v^{\prime}\right)$ times bubble monomials in $\Pi_{\lambda}$.

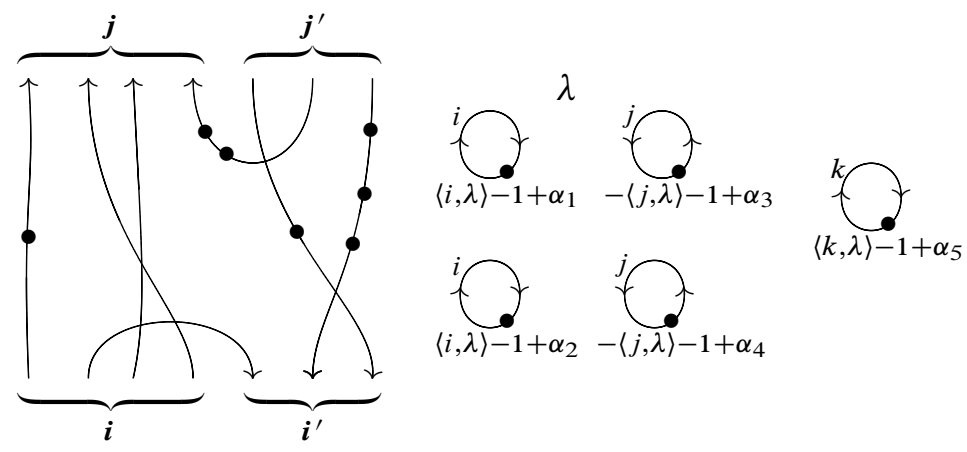

Let $\mathcal{I}_{v,-v^{\prime}, \lambda}$ be the subspace spanned by diagrams which contain a U-turn, i.e., an arc with both endpoints on $\mathbb{R} \times\{1\}$ or on $\mathbb{R} \times\{0\}$.

Proposition 3.17. $\mathcal{I}_{v,-v^{\prime}, \lambda}$ is a 2-sided homogeneous ideal of $\operatorname{END} u\left(\mathcal{E}_{v,-v^{\prime}} \mathbf{1}_{\lambda}\right)$ which does not depend on choices of minimal diagrams for pairings.

Proof. Left to the reader.

Denote by $R_{v,-v^{\prime}, \lambda}:=\operatorname{END} u\left(\mathcal{E}_{v,-v^{\prime}} \mathbf{1}_{\lambda}\right) / \mathcal{I}_{v,-v^{\prime}, \lambda}$ the graded quotient ring, and by $\beta$ the quotient map. There is a homomorphism

$$
\alpha: R(v) \otimes_{\mathbb{k}} R\left(v^{\prime}\right) \otimes_{\mathbb{k}} \Pi_{\lambda} \longrightarrow \operatorname{END} u\left(\varepsilon_{v,-v^{\prime}} \mathbf{1}_{\lambda}\right)
$$

given by placing diagrams representing elements in $R(v), R\left(v^{\prime}\right), \Pi_{\lambda}$ in parallel

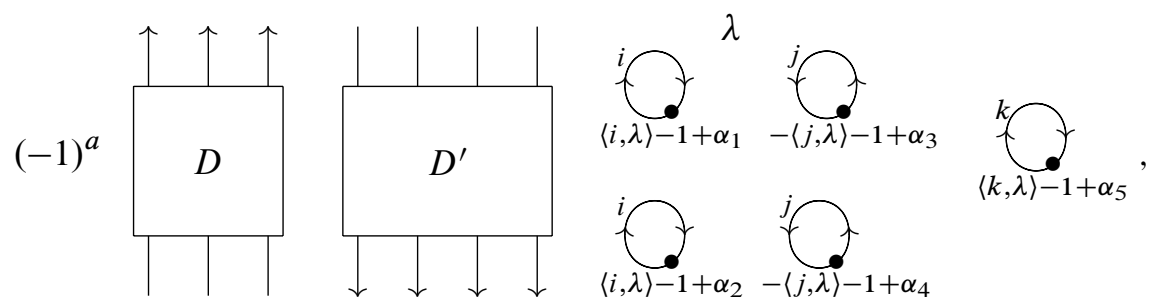

orienting diagrams for $R(v)$ upwards and diagrams for $R\left(v^{\prime}\right)$ downwards, and multiplying by $(-1)^{a}$, where $a$ is the number of crossings in $D^{\prime}$ of equally labelled strands. 
The composition $\beta \alpha$ of this homomorphism with the quotient map is surjective. The diagram below contains an exact sequence of a ring, its 2 -sided ideal, and the quotient ring:

$$
0 \longrightarrow \mathcal{I}_{v,-v^{\prime}, \lambda} \longrightarrow \operatorname{END}_{u}\left(\mathscr{E}_{v,-v^{\prime}} \mathbf{1}_{\lambda}\right) \underset{\beta}{\longrightarrow} R_{v,-v^{\prime}, \lambda} \longrightarrow 0 .
$$

Remark 3.18. If the graphical calculus is non-degenerate, $\beta \alpha$ is an isomorphism, and the sequence splits

$$
0 \longrightarrow \mathcal{I}_{v,-v^{\prime}, \lambda} \longrightarrow \operatorname{END}_{u}\left(\mathcal{E}_{v,-v^{\prime}} \mathbf{1}_{\lambda}\right) \underset{\alpha}{\stackrel{\beta}{\rightleftarrows}} R(v) \otimes R\left(v^{\prime}\right) \otimes \Pi_{\lambda} \longrightarrow 0 .
$$

A split ring homomorphism induces a split exact sequence of Grothendieck groups [28, Section 1.5],

$$
\begin{aligned}
0 \longrightarrow K_{0}\left(\mathcal{I}_{v,-v^{\prime}, \lambda}\right) \longrightarrow K_{0}\left(\mathrm{END}_{u}\left(\mathcal{E}_{v,-v^{\prime}} \mathbf{1}_{\lambda}\right)\right) \\
\stackrel{K_{0}(\beta)}{\longleftarrow} K_{0}\left(R(v) \otimes R\left(v^{\prime}\right) \otimes \Pi_{\lambda}\right) \longrightarrow 0,
\end{aligned}
$$

leading to a canonical decomposition of the middle term as the sum of its two neighbors.

\subsection{Properties and symmetries of 2-category $\mathcal{U}$}

3.3.1. Almost biadjoints. The 1 -morphism $\varepsilon_{+i} \mathbf{1}_{\lambda}$ does not have a simultaneous left and right adjoint $\mathcal{E}_{-i} \mathbf{1}_{\lambda+i_{X}}$ because the units and counits which realize these biadjoints in $\mathcal{U}^{*}$ are not degree-preserving. However, if we shift $\mathcal{E}_{-i} \mathbf{1}_{\lambda+i_{X}}$ by $\left\{-c_{+i, \lambda}\right\}$, then the unit and counit for the adjunction $\mathcal{E}_{+i} \mathbf{1}_{\lambda} \dashv \mathcal{E}_{-i} \mathbf{1}_{\lambda+i_{X}}\left\{-c_{+i, \lambda}\right\}$ become degree-preserving. More generally, we have $\mathcal{E}_{+i} \mathbf{1}_{\lambda}\{t\} \dashv \mathcal{E}_{-i} \mathbf{1}_{\lambda+i_{X}}\left\{-c_{+i, \lambda}-t\right\}$ in $\mathcal{U}$ since the units and counits have degree

$$
\begin{aligned}
& \operatorname{deg}(\underbrace{-i}_{i} \lambda_{\lambda}^{+i\left\{t-c_{+i, \lambda}-t\right\}})=c_{+i, \lambda}+\left(-c_{+i, \lambda}\right)=0, \\
& \left.\operatorname{deg}(\overbrace{+i}^{i} \gamma_{-i\left\{t-c_{+i, \lambda}-t\right\}}^{\lambda+i_{X}})=\left(c_{-i, \lambda+i_{X}}\right)\right)-\left(-c_{+i, \lambda}\right)=0
\end{aligned}
$$

and still satisfy the zigzag identities. Similarly, $\mathcal{E}_{+i} \mathbf{1}_{\lambda}\{t\}$ possesses a left adjoint $\mathcal{E}_{-i} \mathbf{1}_{\lambda+i_{X}}\left\{c_{+i, \lambda}-t\right\}$ in $\mathcal{U}$. One can check that with these shifts the units and counits 
of the adjunction $\mathcal{E}_{-i} \mathbf{1}_{\lambda+i_{X}}\left\{c_{+i, \lambda}-t\right\} \dashv \mathcal{E}_{+i} \mathbf{1}_{\lambda}\{t\}$ become degree zero and are compatible with the zigzag identities.

The left adjoint $\mathcal{E}_{-i} \mathbf{1}_{\lambda+i_{X}}\left\{c_{+i, \lambda}-t\right\}$ and the right adjoint $\mathcal{E}_{-i} \mathbf{1}_{\lambda+i_{X}}\left\{-c_{+i, \lambda}-t\right\}$ of $\mathcal{E}_{+i} \mathbf{1}_{\lambda}\{t\}$ only differ by a shift. We call morphisms with this property almost biadjoint. This situation is familiar to those studying derived categories of coherent sheaves on Calabi-Yau manifolds. Functors with these properties are called 'almost Frobenius functors' in [15] where several other examples of this phenomenon are also given.

It is then clear that $\mathscr{E}_{+i} \mathbf{1}_{\lambda}\{t\}$ and $\mathcal{E}_{-i} \mathbf{1}_{\lambda}\{t\}$ have almost biadjoints in $U$ for all $t \in \mathbb{Z}$ and $\lambda \in X$ with

$$
\begin{aligned}
& \mathbf{1}_{\lambda} \varepsilon_{-i} \mathbf{1}_{\lambda+i_{X}}\left\{c_{+i, \lambda}-t\right\} \dashv \mathbf{1}_{\lambda+i_{X}} \varepsilon_{+i} \mathbf{1}_{\lambda}\{t\} \dashv \mathbf{1}_{\lambda} \mathcal{E}_{-i} \mathbf{1}_{\lambda+i_{X}}\left\{-c_{+i, \lambda}-t\right\}, \\
& \mathbf{1}_{\lambda} \varepsilon_{+i} \mathbf{1}_{\lambda-i_{X}}\left\{c_{-i, \lambda}-t\right\} \dashv \mathbf{1}_{\lambda-i_{X}} \mathcal{E}_{-i} \mathbf{1}_{\lambda}\{t\} \dashv \mathbf{1}_{\lambda} \varepsilon_{+i} \mathbf{1}_{\lambda-i_{X}}\left\{-c_{-i, \lambda}-t\right\}
\end{aligned}
$$

Every 1-morphism in $U$ is a direct sum of composites of $\mathcal{E}_{+i} \mathbf{1}_{\lambda}\{t\}$ 's and $\mathcal{E}_{-i} \mathbf{1}_{\lambda}\{t\}$ 's together with identities; by composing adjunctions as explained in [21, Section 5.5], the right and left adjoints of $\mathcal{E}_{\boldsymbol{i}} \mathbf{1}_{\lambda}\{t\}$ can be computed. Thus, it is clear that all 1-morphisms in $U$ have almost biadjoints.

3.3.2. Positivity of bubbles. The degree of any closed diagram must be greater than or equal to zero. In particular, $\mathcal{U}\left(\mathbf{1}_{\lambda}, \mathbf{1}_{\lambda}\{t\}\right)=0$ if $t>0$, and $\mathcal{U}\left(\mathbf{1}_{\lambda}, \mathbf{1}_{\lambda}\right)$ is at most 1 -dimensional (isomorphic to $\mathbb{k}$ if the calculus is non-degenerate).

3.3.3. Symmetries of $\boldsymbol{u}$. We denote by $\boldsymbol{U}^{\text {op }}$ the 2-category with the same objects as $U$ but the 1-morphisms reversed. The direction of the 2-morphisms remain fixed. The 2-category $U^{\text {co }}$ has the same objects and 1-morphism as $U$, but the directions of the 2-morphisms is reversed. That is, $\mathcal{U}^{\mathrm{co}}(x, y)=U(y, x)$ for 1-morphisms $x$ and $y$. Finally, $\mathcal{U}^{\text {coop }}$ denotes the 2-category with the same objects as $\mathcal{U}$, but the directions of the 1-morphisms and 2-morphisms have been reversed.

Using the symmetries of the diagrammatic relations imposed on $U$ we construct 2 -functors on the various versions of $\mathcal{U}$. In Proposition 3.28 we relate these 2 -functors to various $\mathbb{Z}\left[q, q^{-1}\right]$-(anti)linear (anti)automorphisms of the algebra $\dot{\mathbf{U}}$. The various forms of contravariant behaviour for 2-functors on $\mathcal{U}$ translate into properties of the corresponding homomorphism in $\dot{\mathbf{U}}$ as the following table summarizes.

\begin{tabular}{|l|l|}
\hline 2-functors & Algebra maps \\
\hline \hline $\mathcal{U} \rightarrow \mathcal{U}$ & $\mathbb{Z}\left[q, q^{-1}\right]$-linear homomorphisms \\
$\mathcal{U} \rightarrow \mathcal{U}^{\text {op }}$ & $\mathbb{Z}\left[q, q^{-1}\right]$-linear antihomomorphisms \\
$\mathcal{U} \rightarrow \mathcal{U}^{\text {co }}$ & $\mathbb{Z}\left[q, q^{-1}\right]$-antilinear homomorphisms \\
$\mathcal{U} \rightarrow \mathcal{U}^{\text {coop }}$ & $\mathbb{Z}\left[q, q^{-1}\right]$-antilinear antihomomorphisms \\
\hline
\end{tabular}


Rescale, invert the orientation, and send $\lambda \mapsto-\lambda$ : Consider the operation on the diagrammatic calculus that rescales the $i i$-crossing $\chi_{i, i, \lambda} \mapsto-\chi_{i, i, \lambda}$ for all $i \in I$ and $\lambda \in X$, inverts the orientation of each strand and sends $\lambda \mapsto-\lambda$ :

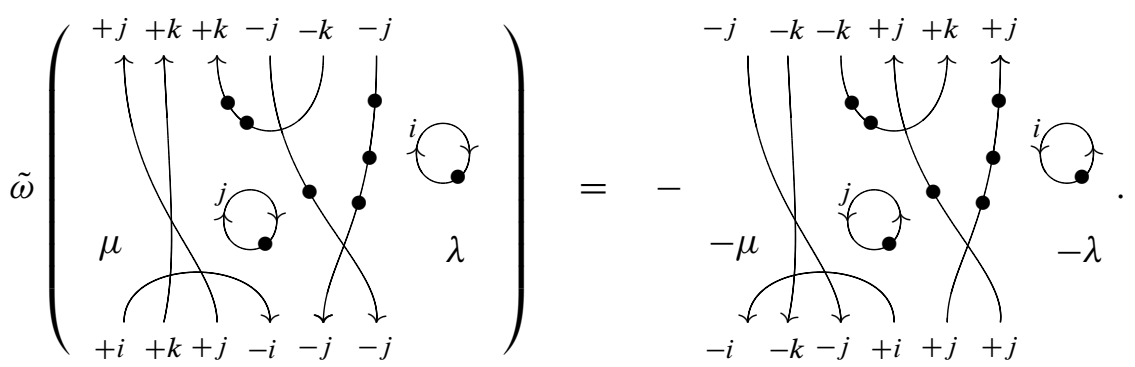

This transformation preserves the degree of a diagram, so by extending to sums of diagrams we get a 2 -functor $\tilde{\omega}: \mathcal{U} \rightarrow \mathcal{U}$ given by

$$
\tilde{\omega}: U \rightarrow U, \quad \lambda \mapsto-\lambda, \quad \mathbf{1}_{\mu} \mathcal{E}_{\boldsymbol{i}} \mathbf{1}_{\lambda}\{t\} \mapsto \mathbf{1}_{-\mu} \mathcal{E}_{-\boldsymbol{i}} \mathbf{1}_{-\lambda}\{t\} .
$$

It is straight forward to check that $\tilde{\omega}$ is a strict 2-functor. In fact, it is a 2-isomorphism since its square is the identity.

Rescale, reflect across the $\boldsymbol{y}$-axis, and send $\lambda \mapsto-\lambda$ : The operation on diagrams that rescales the $i i$-crossing $\chi_{i, i, \lambda} \mapsto-\chi_{i, i, \lambda}$ for all $i \in I$ and $\lambda \in X$, reflects a diagram across the $y$-axis, sends $\lambda$ to $-\lambda$ and leaves invariant the relations on the 2-morphisms of $\mathcal{U}$. This operation

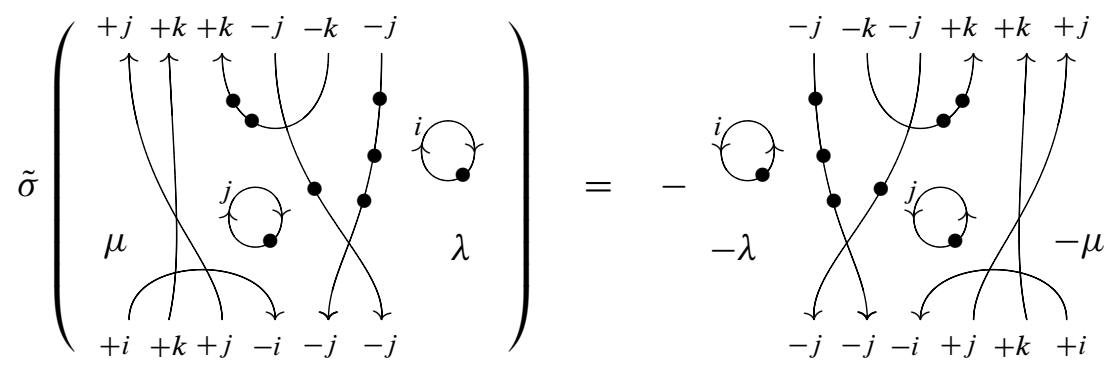

is contravariant for composition of 1-morphisms, covariant for composition of 2morphisms, and preserves the degree of a diagram. Hence, this symmetry gives a 2-isomorphism

$$
\begin{aligned}
\tilde{\sigma}: \mathcal{U} & \rightarrow \mathcal{U}^{\mathrm{op}}, \\
\lambda & \mapsto-\lambda, \\
\mathbf{1}_{\mu} \varepsilon_{s_{1}} \varepsilon_{s_{2}} \ldots \mathcal{E}_{s_{m-1}} \varepsilon_{s_{m}} \mathbf{1}_{\lambda}\{t\} & \mapsto \mathbf{1}_{-\lambda} \varepsilon_{s_{m}} \varepsilon_{s_{m-1}} \ldots \mathcal{E}_{s_{2}} \varepsilon_{s_{1}} \mathbf{1}_{-\mu}\{t\},
\end{aligned}
$$

and on 2-morphisms $\tilde{\sigma}$ maps linear combinations of diagrams to the linear combination of the diagrams obtained by applying the above transformation to each summand. The relations on $\mathcal{U}$ are symmetric under this transformation, and $\tilde{\sigma}$ is a 2 -functor. The square of $\tilde{\sigma}$ is the identity. 
Reflect across the $\mathbf{x}$-axis and invert orientation: Here we are careful to keep track of what happens to the shifts of sources and targets:

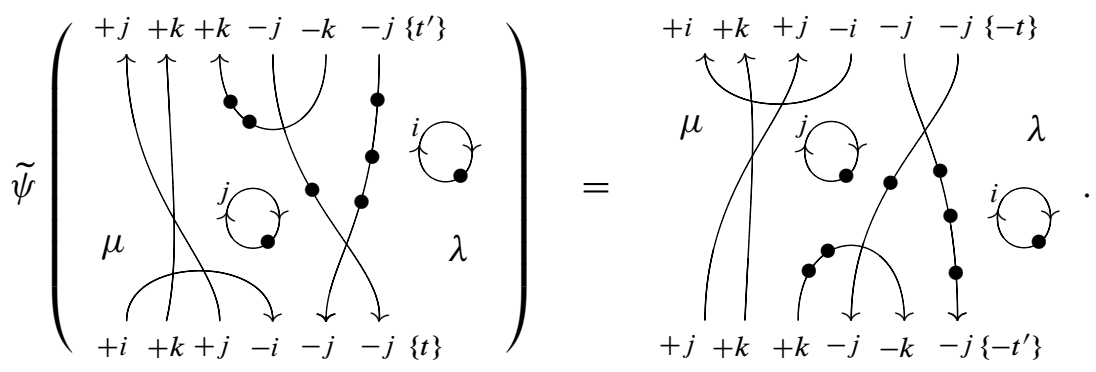

The degree shifts on the right-hand side are required for this transformation to preserve the degree of a diagram. This transformation preserves the order of composition of 1morphisms, but is contravariant with respect to composition of 2-morphisms. Hence, by extending this transformation to sums of diagrams we get a 2 -isomorphism given by

$$
\tilde{\psi}: U \rightarrow U^{\mathrm{co}}, \quad \lambda \mapsto \lambda, \quad \mathbf{1}_{\mu} \mathcal{E}_{i} \mathbf{1}_{\lambda}\{t\} \mapsto \mathbf{1}_{\mu} \mathcal{E}_{i} \mathbf{1}_{\lambda}\{-t\},
$$

and on 2-morphisms $\tilde{\psi}$ reflects the diagrams representing summands across the $x$ axis and inverts the orientation. Again, the relations on $\mathcal{U}$ possess this symmetry so it is not difficult to check that $\widetilde{\psi}$ is a 2 -functor. Furthermore, it is clear that $\widetilde{\psi}$ is invertible since its square is the identity.

It is easy to see that these 2-functors commute with each other 'on-the-nose'. That is, we have equalities

$$
\tilde{\omega} \tilde{\sigma}=\tilde{\omega} \tilde{\sigma}, \quad \tilde{\sigma} \tilde{\psi}=\tilde{\psi} \tilde{\sigma}, \quad \tilde{\omega} \tilde{\psi}=\tilde{\psi} \tilde{\omega} .
$$

The composite 2-functor $\tilde{\psi} \tilde{\omega} \tilde{\sigma}$ is given by

$$
\begin{aligned}
\tilde{\psi} \tilde{\omega} \tilde{\sigma}: \mathcal{U} & \rightarrow u^{\text {coop }}, \\
\lambda & \mapsto \lambda, \\
\mathbf{1}_{\mu} \varepsilon_{s_{1}} \mathcal{E}_{s_{2}} \ldots \mathcal{E}_{s_{m-1}} \mathcal{E}_{s_{m}} \mathbf{1}_{\lambda}\{t\} & \mapsto \mathbf{1}_{\lambda} \mathcal{E}_{-s_{m}} \varepsilon_{-s_{m-1}} \ldots \mathcal{E}_{-s_{2}} \mathcal{E}_{-s_{1}} \mathbf{1}_{\mu}\{-t\},
\end{aligned}
$$

and is given on 2-morphisms by rotating diagrams by $180^{\circ}$.

The following transformation only differs from $\tilde{\psi} \tilde{\omega} \tilde{\sigma}$ by a shift and is given by taking adjoints.

Rotation by $180^{\circ}$ (taking right adjoints). This transformation is a bit more subtle because it uses the almost biadjoint structure of $U$, in particular, the calculus of mates (see [21, Section 4.3]). For each $\mathbf{1}_{\mu} x \mathbf{1}_{\lambda} \in U$ denote its right adjoint by $\mathbf{1}_{\lambda} y \mathbf{1}_{\mu}$. The symmetry of rotation by $180^{\circ}$ can also be realized by the 2 -functor that sends a 1-morphism $\mathbf{1}_{\mu} x \mathbf{1}_{\lambda}$ to its right adjoint $\mathbf{1}_{\lambda} y \mathbf{1}_{\mu}$ and each 2-morphism $\zeta: \mathbf{1}_{\mu} x \mathbf{1}_{\lambda} \Rightarrow$ 
$\mathbf{1}_{\mu} x^{\prime} \mathbf{1}_{\lambda}$ to its mate under the adjunctions $\mathbf{1}_{\mu} x \mathbf{1}_{\lambda} \dashv \mathbf{1}_{\lambda} y \mathbf{1}_{\mu}$ and $\mathbf{1}_{\mu} x^{\prime} \mathbf{1}_{\lambda} \dashv \mathbf{1}_{\lambda} y^{\prime} \mathbf{1}_{\mu}$. That is, $\zeta$ is mapped to its right dual $\zeta^{*}$. Pictorially,

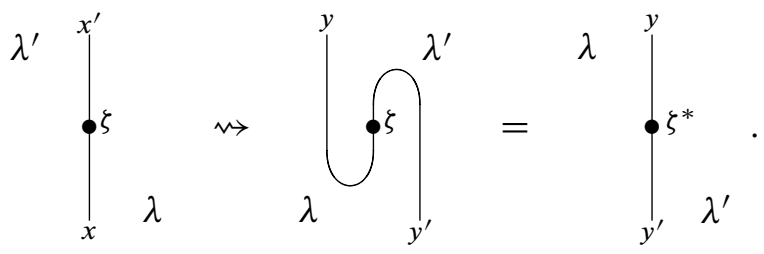

This transformation is contravariant with respect to composition of 1-morphisms and 2-morphisms. We get a 2-functor

$$
\begin{aligned}
\tilde{\tau}: U & \rightarrow u^{\text {coop }} \\
\lambda & \mapsto \lambda, \\
\mathbf{1}_{\mu} \varepsilon_{s_{1}} \varepsilon_{s_{2}} \ldots \mathcal{E}_{s_{m-1}} \varepsilon_{s_{m}} \mathbf{1}_{\lambda}\{t\} & \mapsto \mathbf{1}_{\lambda} \mathcal{E}_{-s_{m}} \mathcal{E}_{-s_{m-1}} \ldots \mathcal{E}_{-s_{2}} \mathcal{E}_{-s_{1}} \mathbf{1}_{\mu}\left\{-t+t^{\prime}\right\}, \\
\zeta & \mapsto \zeta^{*},
\end{aligned}
$$

where the degree shift $t^{\prime}$ for the right adjoint $\mathbf{1}_{\lambda} \mathcal{E}_{-s_{m}} \mathcal{E}_{-s_{m-1}} \ldots \mathcal{E}_{-s_{2}} \mathcal{E}_{-s_{1}} \mathbf{1}_{\mu}\{-t+$ $\left.t^{\prime \prime}\right\}$, determined by (3.25), ensures that $\tilde{\tau}$ is degree-preserving. Inspection of the relations for $U$ will reveal that they are invariant under this transformation so that $\tilde{\tau}$ is a 2 -functor.

We can define an inverse for $\tilde{\tau}$ given by taking left adjoints. We record this 2-morphism here.

$$
\begin{aligned}
\tilde{\tau}^{-1}: \mathcal{U} & \rightarrow \mathcal{u}^{\text {coop }}, \\
\lambda & \mapsto \lambda, \\
\mathbf{1}_{\mu} \varepsilon_{s_{1}} \varepsilon_{s_{2}} \ldots \mathcal{E}_{s_{m-1}} \varepsilon_{s_{m}} \mathbf{1}_{\lambda}\{t\} & \mapsto \mathbf{1}_{\lambda} \mathcal{E}_{-s_{m}} \mathcal{E}_{-s_{m-1}} \ldots \mathcal{E}_{-s_{2}} \mathcal{E}_{-s_{1}} \mathbf{1}_{\mu}\left\{-t+t^{\prime \prime}\right\}, \\
\zeta & \mapsto{ }^{*} \zeta,
\end{aligned}
$$

with degree shift $t^{\prime \prime}$ determined from (3.25) and the left dual $* \zeta$ of the 2-morphism $\zeta$ defined in [21, Section 4.3].

Remark 3.19. The composition $\tilde{\tau} \tilde{\psi} \tilde{\omega} \tilde{\sigma}: U \rightarrow U$ gives 2 -isomorphism that fixes all diagrams and only effects the grading shifts.

Remark 3.20. There are degree zero isomorphisms of graded $\mathbb{k}$-vector spaces,

$$
\begin{aligned}
& U^{*}(f x, y) \rightarrow U^{*}(x, \tilde{\tau}(f) y), \\
& u^{*}(x, g y) \rightarrow u^{*}\left(\tilde{\tau}^{-1}(g) x, y\right),
\end{aligned}
$$

for all 1-morphisms $f, g, x, y$ in $U^{*}$, defined at the end of Section 3.1.1. 
3.4. Karoubi envelope, $\dot{u}$, and 2 -representations. The Karoubi envelope $\operatorname{Kar}(\mathcal{C})$ of a category $\zeta$ is an enlargement of the category $\zeta$ in which all idempotents split (see [21, Section 9] and references therein). There is a fully faithful functor $\mathcal{C} \rightarrow$ $\operatorname{Kar}(\mathcal{C})$ that is universal with respect to functors which split idempotents in $\mathcal{C}$. This means that if $F: \mathscr{C} \rightarrow \mathscr{D}$ is any functor where all idempotents split in $\mathscr{D}$, then $F$ extends uniquely (up to isomorphism) to a functor $\widetilde{F}: \operatorname{Kar}(\mathcal{C}) \rightarrow \mathscr{D}$ (see for example [4], Proposition 6.5.9). Furthermore, for any functor $G: \mathcal{C} \rightarrow \mathscr{D}$ and a natural transformation $\alpha: F \Rightarrow G, \alpha$ extends uniquely to a natural transformation $\tilde{\alpha}: \widetilde{F} \Rightarrow \widetilde{G}$. When $\mathcal{C}$ is additive the inclusion $\mathcal{C} \rightarrow \operatorname{Kar}(\ell)$ is an additive functor.

Definition 3.21. Define the additive $\mathbb{k}$-linear 2-category $\dot{\mathcal{U}}$ to have the same objects as $U$ and hom additive $\mathbb{k}$-linear categories given by $\dot{U}\left(\lambda, \lambda^{\prime}\right)=\operatorname{Kar}\left(U\left(\lambda, \lambda^{\prime}\right)\right)$. The fully-faithful additive $k$-linear functors $\mathcal{U}\left(\lambda, \lambda^{\prime}\right) \rightarrow \dot{U}\left(\lambda, \lambda^{\prime}\right)$ combine to form an additive $\mathbb{k}$-linear 2-functor $U \rightarrow \dot{U}$ universal with respect to splitting idempotents in the hom categories $\dot{U}\left(\lambda, \lambda^{\prime}\right)$. The composition functor $\dot{U}\left(\lambda, \lambda^{\prime}\right) \times \dot{U}\left(\lambda^{\prime}, \lambda^{\prime \prime}\right) \rightarrow$ $\dot{U}\left(\lambda, \lambda^{\prime \prime}\right)$ is induced by the universal property of the Karoubi envelope from the composition functor for $U$. The 2-category $\dot{U}$ has graded 2-homs given by

$$
\operatorname{HOM}_{\dot{u}}(x, y):=\bigoplus_{t \in \mathbb{Z}} \operatorname{Hom}_{\dot{u}}(x\{t\}, y) .
$$

Definition 3.22. A 2-representation of $U^{*}$ is a (weak) graded additive $\mathbb{k}$-linear 2functor $\Psi^{*}: \mathcal{U}^{*} \rightarrow \mathcal{M}^{*}$, where $\mathcal{M}^{*}$ is a graded additive $\mathbb{k}$-linear 2-category with a translation.

A 2-representation of $U$ is an additive $\mathbb{k}$-linear 2-functor $\Psi: U \rightarrow \mathcal{M}$ that respects the grading. This happens when there is an additive $\mathbb{k}$-linear 2-functor $\mathcal{M} \rightarrow \mathcal{M}^{*}$, with $\mathcal{M}^{*}$ a graded additive $\mathbb{k}$-linear 2-category, making the diagram

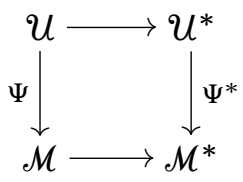

weakly commutative. Thus, to study 2 -representation of $U$ it suffices to study 2 representation of $U^{*}$ and then restrict to degree-preserving 2-morphisms.

A 2-representation of $\dot{U}$ is an additive $\mathbb{k}$-linear 2-functor $\dot{\Psi}: \dot{U} \rightarrow \mathcal{M}$ that respects the grading.

Denote by $\mathcal{M}$ a 2-category as above in which idempotents split. Any 2-representation $\Psi^{*}: \mathcal{U}^{*} \rightarrow \mathcal{M}^{*}$ gives a unique (up to isomorphism) 2-representation $\dot{\Psi}: \dot{U} \rightarrow$ $\mathcal{M}$. The 2 -functor $\dot{\Psi}$ is obtained from $\Psi^{*}$ by restricting to the degree-preserving 2 morphisms of $U^{*}$ and using the universal property of the Karoubi envelope. This is 
illustrated schematically below.

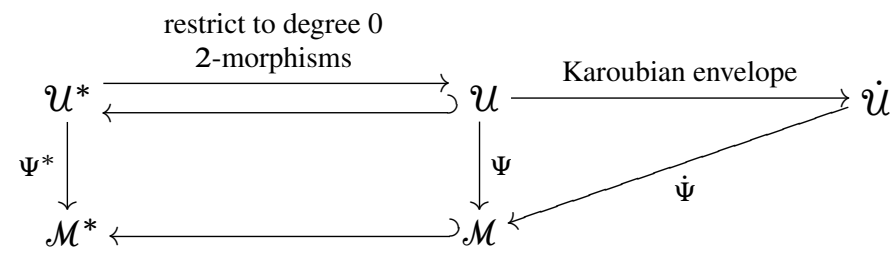

Remark 3.23. The 2-functors $\tilde{\omega}, \tilde{\sigma}, \tilde{\psi}, \tilde{\tau}$ on $U$ extend to 2 -functors on $\dot{U}$, for which we use the same notations. For example,

$$
\begin{aligned}
& \tilde{\omega}: \dot{u} \rightarrow \dot{u}, \\
& \lambda \mapsto-\lambda, \\
& \left(\mathcal{E}_{\boldsymbol{i}} \mathbf{1}_{\lambda}\{t\}, e\right) \mapsto\left(\tilde{\omega}\left(\mathcal{E}_{\boldsymbol{i}} \mathbf{1}_{\lambda}\{t\}\right), \tilde{\omega}(e)\right), \\
& \zeta \mapsto \tilde{\omega}(\zeta)
\end{aligned}
$$

and the other 2-morphisms $\tilde{\sigma}, \tilde{\psi}$, and $\tilde{\tau}$ are defined analogously. In particular, each 1-morphism in $\dot{U}$ has left and right adjoints.

Multigrading. The multigrading introduced at the end of [17] on rings $R(v)$ extends to a multigrading on each hom space $\mathcal{U}^{*}\left(\mathcal{E}_{\boldsymbol{i}} \mathbf{1}_{\lambda}, \boldsymbol{E}_{\boldsymbol{j}} \mathbf{1}_{\lambda}\right)$. The Karoubian envelope of the corresponding multigraded 2-category should categorify the multi-parameter deformation of ${ }_{\mathcal{A}} \dot{\mathbf{U}}$.

3.5. Direct sum decompositions. Recall that $\mathcal{E}_{\nu} \mathbf{1}_{\lambda}$ is the direct sum of $\mathcal{E}_{\boldsymbol{i}} \mathbf{1}_{\lambda}$ over all $i \in \operatorname{Seq}(v):$

$$
\mathcal{E}_{\nu} \mathbf{1}_{\lambda}:=\bigoplus_{i \in \operatorname{Seq}(\nu)} \varepsilon_{i} \mathbf{1}_{\lambda}
$$

Due to the existence of the homomorphism $\varphi_{\nu, \lambda}$ in the formula (3.22) any degree 0 idempotent $e$ of $R(v)$ gives rise to the idempotent $\varphi_{\nu, \lambda}(e)$ of $\varepsilon_{\nu} \mathbf{1}_{\lambda}$ and to the 1-morphism $\left(\varepsilon_{\nu} \mathbf{1}_{\lambda}, \varphi_{\nu, \lambda}(e)\right)$ of $\dot{U}$.

Introduce idempotents

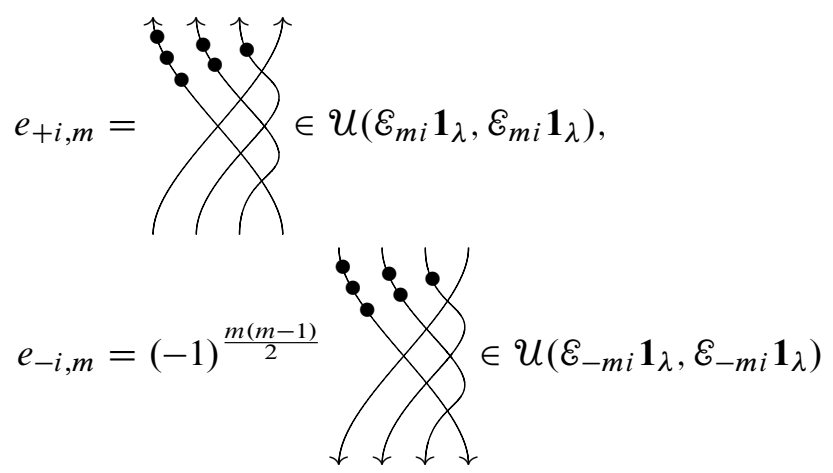


similar to the idempotent $e_{i, m}$ in [16, Section 2.2] and [17]. Define 1-morphisms $\mathcal{E}_{+i}(m) \mathbf{1}_{\lambda}$ and $\mathcal{E}_{-i(m)} \mathbf{1}_{\lambda}$ in $\dot{U}$ by

$$
\begin{aligned}
& \mathcal{E}_{+i(m)} \mathbf{1}_{\lambda}:=\left(\mathcal{E}_{+i m} \mathbf{1}_{\lambda}, e_{+i, m}\right)\left\{\frac{m(1-m)}{2} \frac{i \cdot i}{2}\right\}, \\
& \mathcal{E}_{-i^{(m)}} \mathbf{1}_{\lambda}:=\left(\mathcal{E}_{-i m} \mathbf{1}_{\lambda}, e_{-i, m}\right)\left\{\frac{m(1-m)}{2} \frac{i \cdot i}{2}\right\} .
\end{aligned}
$$

As in [16], [17], we have direct sum decompositions

$$
\mathcal{E}_{+i m} \mathbf{1}_{\lambda} \cong\left(\mathcal{E}_{+i}(m) \mathbf{1}_{\lambda}\right)^{\oplus[m]_{i} !}, \quad \mathcal{E}_{-i m} \mathbf{1}_{\lambda} \cong\left(\mathcal{E}_{-i(m)} \mathbf{1}_{\lambda}\right)^{\oplus[m]_{i} !} .
$$

For any divided power sequence $\boldsymbol{i}=\left(\varepsilon_{1} i_{1}^{\left(a_{1}\right)}, \varepsilon_{2} i_{2}^{\left(a_{2}\right)}, \ldots, \varepsilon_{m} i_{m}^{\left(a_{m}\right)}\right)$ define

$$
\mathcal{E}_{\boldsymbol{i}} \mathbf{1}_{\lambda}:=\left(\mathcal{E}_{\hat{\boldsymbol{i}}} \mathbf{1}_{\lambda}, e_{\boldsymbol{i}}\right)
$$

where $\hat{i}$ is the sequence

$$
\begin{gathered}
\left(\varepsilon_{1} i_{1}, \ldots, \varepsilon_{1} i_{1}, \varepsilon_{2} i_{2}, \ldots \varepsilon_{2} i_{2}, \ldots, \varepsilon_{m} i_{m} \ldots, \varepsilon_{m} i_{m}\right) \\
=\left(\left(\varepsilon_{1} i_{1}\right)^{a_{1}}\left(\varepsilon_{2} i_{2}\right)^{a_{2}} \ldots\left(\varepsilon_{m} i_{m}\right)^{a_{m}}\right),
\end{gathered}
$$

with term $\varepsilon_{1} i_{1}$ repeating $a_{1}$ times, term $\varepsilon_{2} i_{2}$ repeating $a_{2}$ times, etc., and

$$
e_{\boldsymbol{i}}=e_{\varepsilon_{1} i_{1}, a_{1}} \cdot e_{\varepsilon_{2} i_{2}, a_{2}} \ldots e_{\varepsilon_{m} i_{m}, a_{m}}
$$

is the horizontal product of idempotents.

When interested in only one part of a sequence $\boldsymbol{i}$, we write $\ldots \boldsymbol{i}^{\prime \prime} \ldots$ instead of

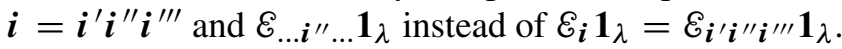

Proposition 3.24. For each $i, j \in I, i \neq j$, and $\lambda \in X$ there are 2-isomorphisms of 1-morphisms in $\dot{U}$ :

$$
\begin{aligned}
& \bigoplus_{a=0}^{\left\lfloor\frac{d+1}{2}\right\rfloor} \mathcal{E}_{\ldots+i(2 a)}+j+i^{(d+1-2 a)} \ldots \mathbf{1}_{\lambda} \cong \bigoplus_{a=0}^{\left\lfloor\frac{d}{2}\right\rfloor} \mathcal{E}_{\ldots+i^{(2 a+1)}+j+i^{(d-2 a)} \ldots} \mathbf{1}_{\lambda}, \\
& \left\lfloor\frac{d+1}{2}\right\rfloor \\
& \left\lfloor\frac{d}{2}\right\rfloor \\
& \bigoplus_{a=0}^{2} \mathcal{E}_{\ldots-i(2 a)}-j-i(d+1-2 a) \ldots \mathbf{1}_{\lambda} \cong \bigoplus_{a=0} \mathcal{E}_{\ldots-i(2 a+1)}-j-i^{(d-2 a)} \ldots \mathbf{1}_{\lambda},
\end{aligned}
$$

where $d=d_{i j}=-\left\langle i, j_{X}\right\rangle$.

Proof. These isomorphisms follow from categorified quantum Serre relations [16, Proposition 2.13] and [17, Proposition 6] between idempotents in rings $R(v)$, via homomorphisms $\varphi_{v, \lambda}$ and $\varphi_{-v, \lambda}$. 
Proposition 3.25. For each $i \in I, \lambda \in X$ there are 2-isomorphisms in $\dot{u}$

$$
\begin{array}{ll}
\mathcal{E}_{\boldsymbol{i}^{\prime}+i-i \boldsymbol{i}^{\prime \prime}} \mathbf{1}_{\lambda} \cong \mathcal{E}_{\boldsymbol{i}^{\prime}-i+i \boldsymbol{i}^{\prime \prime}} \mathbf{1}_{\lambda} \oplus_{[\langle i, \mu\rangle]_{i}} \mathcal{E}_{\boldsymbol{i}^{\prime} \boldsymbol{i}^{\prime \prime}} \mathbf{1}_{\lambda} \quad \text { if }\left\langle i, \lambda+\boldsymbol{i}_{X}^{\prime \prime}\right\rangle \geq 0, \\
\mathcal{E}_{\boldsymbol{i}^{\prime}-i+i \boldsymbol{i}^{\prime \prime}} \mathbf{1}_{\lambda} \cong \mathcal{E}_{\boldsymbol{i}^{\prime}+i-i \boldsymbol{i}^{\prime \prime}} \mathbf{1}_{\lambda} \oplus_{-[\langle i, \mu\rangle]_{i}} \mathcal{E}_{\boldsymbol{i}^{\prime} \boldsymbol{i}^{\prime \prime}} \mathbf{1}_{\lambda} \quad \text { if }\left\langle i, \lambda+\boldsymbol{i}_{X}^{\prime \prime}\right\rangle \leq 0,
\end{array}
$$

where $\mu=\lambda+i_{X}^{\prime \prime}$.

Proof. Set $\lambda+\boldsymbol{i}_{X}^{\prime \prime}=\mu$. The decomposition $\left.\mathcal{E}_{\boldsymbol{i}^{\prime}+i-i \boldsymbol{i}^{\prime \prime}} \mathbf{1}_{\lambda} \cong \mathcal{E}_{\boldsymbol{i}^{\prime}-i+i \boldsymbol{i}^{\prime \prime}} \mathbf{1}_{\lambda} \oplus_{[\langle i, \mu\rangle}\right]_{i}$ $\mathcal{E}_{\boldsymbol{i}^{\prime} \boldsymbol{i}^{\prime \prime}} \mathbf{1}_{\lambda}$ for $\langle i, \mu\rangle \geq 0$ is given by 2 -morphisms in $\mathcal{U}$

$$
\begin{array}{r}
\alpha: \mathcal{E}_{\boldsymbol{i}^{\prime}+i-i \boldsymbol{i}^{\prime \prime}} \mathbf{1}_{\lambda} \rightarrow \mathcal{E}_{\boldsymbol{i}^{\prime}-i+i \boldsymbol{i}^{\prime \prime}} \mathbf{1}_{\lambda} \oplus_{[\langle i, \mu\rangle]_{i}} \mathcal{E}_{\boldsymbol{i}^{\prime} \boldsymbol{i}^{\prime \prime}} \mathbf{1}_{\lambda}, \\
\alpha^{-1}: \mathcal{E}_{\boldsymbol{i}^{\prime}-i+i \boldsymbol{i}^{\prime \prime}} \mathbf{1}_{\lambda} \oplus_{[\langle i, \mu\rangle]_{i}} \mathcal{E}_{\boldsymbol{i}^{\prime} \boldsymbol{i}^{\prime \prime}} \mathbf{1}_{\lambda} \rightarrow \mathcal{E}_{\boldsymbol{i}^{\prime}+i-i \boldsymbol{i}^{\prime \prime}} \mathbf{1}_{\lambda},
\end{array}
$$

where $\alpha$ and $\alpha^{-1}$ consist of matrices of diagrams

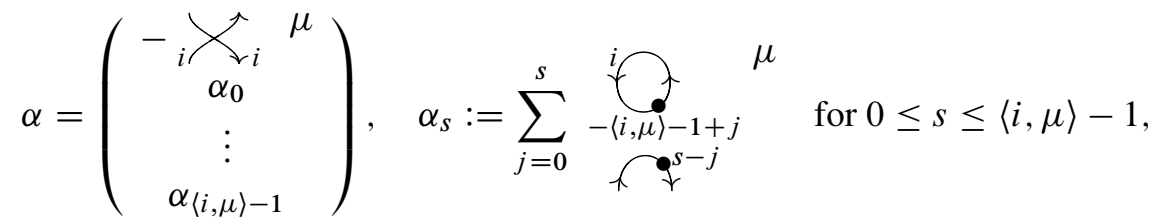

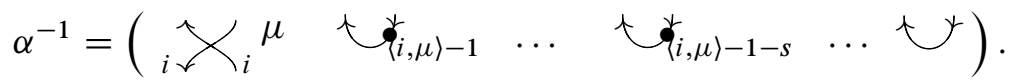

Note that all bubbles that appear in $\alpha_{s}$ above are fake bubbles. One can check that

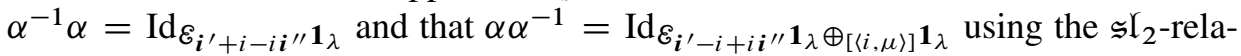
tions (for details see [21]). Here we have taken

$$
\oplus_{[\langle i, \mu\rangle]} \mathbf{1}_{\lambda}=\mathbf{1}_{\lambda}\{1-\langle i, \mu\rangle\} \oplus \cdots \oplus \mathbf{1}_{\lambda}\{2 s+1-\langle i, \mu\rangle\} \oplus \cdots \oplus \mathbf{1}_{\lambda}\{\langle i, \mu\rangle-1\}
$$

so that $\alpha$ and $\alpha^{-1}$ have degree zero. The isomorphism

$$
\mathcal{E}_{\boldsymbol{i}^{\prime}-i+i \boldsymbol{i}^{\prime \prime}} \mathbf{1}_{\lambda} \cong \mathcal{E}_{\boldsymbol{i}^{\prime}+i-i \boldsymbol{i}^{\prime \prime}} \mathbf{1}_{\lambda} \oplus_{-[\langle i, \mu\rangle]_{i}} \mathcal{E}_{\boldsymbol{i}^{\prime} \boldsymbol{i}^{\prime \prime}} \mathbf{1}_{\lambda}
$$

for $\left\langle i, \lambda+i_{X}^{\prime \prime}\right\rangle \leq 0$ is given similarly (see [21]).

Proposition 3.26. For each $i, j \in I, i \neq j, \lambda \in X$ there are 2-isomorphisms

$$
\mathcal{E}_{\ldots+i-j \ldots} \mathbf{1}_{\lambda} \cong \mathcal{E}_{\ldots-j+i \ldots} \mathbf{1}_{\lambda}
$$

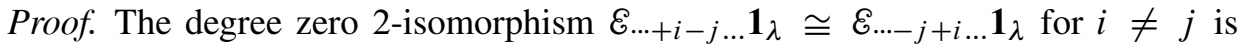
given by maps

$$
\begin{aligned}
& \chi_{i j} \lambda: \varepsilon_{\ldots+i-j \ldots} \mathbf{1}_{\lambda} \rightarrow \mathcal{E}_{\ldots-j+i \ldots} \mathbf{1}_{\lambda}, \\
& \chi_{j \curlyvee} \lambda: \varepsilon_{\ldots-j+i \ldots} \mathbf{1}_{\lambda} \rightarrow \mathcal{E}_{\ldots+i-j \ldots} \mathbf{1}_{\lambda} .
\end{aligned}
$$

To see that these maps are isomorphisms use (3.13). 
3.6. $K_{0}(\dot{u})$ and homomorphism $\gamma . K_{0}(\dot{u})$ can be viewed as a pre-additive category or, alternatively, as an idempotented ring. When thought of as a category, it has objects $\lambda$, over all $\lambda \in X$. The abelian group of morphisms $K_{0}(\dot{U}(\lambda, \mu))$ is defined as the (split) Grothendieck group of the additive category $\dot{U}(\lambda, \mu)$. The split Grothendieck group $K_{0}(\mathcal{A})$ of an additive category $\mathcal{A}$ has generators $[P]$, over all objects $P$ of $\mathcal{A}$, and relations $[P]=\left[P^{\prime}\right]+\left[P^{\prime \prime}\right]$ whenever $P \cong P^{\prime} \oplus P^{\prime \prime}$. In the case of $\dot{U}(\lambda, \mu)$ the generators are $\left[\mathcal{E}_{i} \mathbf{1}_{\lambda}\{t\}, e\right]$, where $\mu=\lambda+\boldsymbol{i}_{X}, t \in \mathbb{Z}$, and

$$
e \in \operatorname{End}_{\dot{u}}\left(\varepsilon_{i} \mathbf{1}_{\lambda}\{t\}\right) \cong \operatorname{End}_{\dot{u}}\left(\mathcal{E}_{\boldsymbol{i}} \mathbf{1}_{\lambda}\right)=\operatorname{End} u\left(\mathcal{E}_{\boldsymbol{i}} \mathbf{1}_{\lambda}\right)
$$

is an idempotent (degree zero idempotent when viewed as an element of the larger ring $\left.\operatorname{END}_{u}\left(\mathcal{E}_{i} \mathbf{1}_{\lambda}\right)\right)$. The defining relations are

$$
\left[\mathcal{E}_{\boldsymbol{i}} \mathbf{1}_{\lambda}\{t\}, e\right]=\left[\mathcal{E}_{\boldsymbol{i}^{\prime}} \mathbf{1}_{\lambda^{\prime}}\left\{t^{\prime}\right\}, e^{\prime}\right]+\left[\mathcal{E}_{\boldsymbol{i}^{\prime \prime}} \mathbf{1}_{\lambda^{\prime \prime}}\left\{t^{\prime \prime}\right\}, e^{\prime \prime}\right]
$$

whenever there is an isomorphism in $\dot{U}(\lambda, \mu)$

$$
\left(\mathcal{E}_{\boldsymbol{i}} \mathbf{1}_{\lambda}\{t\}, e\right) \cong\left(\mathcal{E}_{\boldsymbol{i}^{\prime}} \mathbf{1}_{\lambda^{\prime}}\left\{t^{\prime}\right\}, e^{\prime}\right) \oplus\left(\mathcal{E}_{\boldsymbol{i}^{\prime \prime}} \mathbf{1}_{\lambda^{\prime \prime}}\left\{t^{\prime \prime}\right\}, e^{\prime \prime}\right) .
$$

Moreover, $K_{0}(\dot{U}(\lambda, \mu))$ is a $\mathbb{Z}\left[q, q^{-1}\right]$-module, with multiplication by $q$ coming from the grading shift

$$
\left[\mathscr{E}_{i} \mathbf{1}_{\lambda}\{t+1\}, e\{1\}\right]=q\left[\mathscr{E}_{i} \mathbf{1}_{\lambda}\{t\}, e\right] .
$$

We write $\left[\mathscr{E}_{\boldsymbol{i}} \mathbf{1}_{\lambda}\right]$ instead of $\left[\mathcal{E}_{\boldsymbol{i}} \mathbf{1}_{\lambda}, 1\right]$, where 1 is the identity 2 -morphism of $\mathcal{E}_{\boldsymbol{i}} \mathbf{1}_{\lambda}$.

The space of homs between any two objects in $\dot{U}(\lambda, \mu)$ is a finite-dimensional kvector space. In particular, the Krull-Schmidt decomposition theorem holds, and an indecomposable object of $\dot{U}(\lambda, \mu)$ has the form $\left(\varepsilon_{i} \mathbf{1}_{\lambda}\{t\}, e\right)$ for some mini$\mathrm{mal} /$ primitive idempotent $e$. Any presentation of $1=e_{1}+\cdots+e_{k}$ into the sum of minimal mutually orthogonal idempotents gives rise to a decomposition

$$
\mathcal{E}_{\boldsymbol{i}} \mathbf{1}_{\lambda}\{t\} \cong \bigoplus_{r=1}^{k}\left(\mathcal{E}_{\boldsymbol{i}} \mathbf{1}_{\lambda}\{t\}, e_{r}\right)
$$

into a direct sum of indecomposable objects of $\dot{U}(\lambda, \mu)$. Any object of $\dot{U}(\lambda, \mu)$ has a unique presentation, up to permutation of factors and isomorphisms, as a direct sum of indecomposables. Choose one representative $b$ for each isomorphism class of indecomposables, up to grading shifts, and denote by $\dot{B}(\lambda, \mu)$ the set of these representatives. Then $\{[b]\}_{b}$ is a basis of $K_{0}(\dot{U}(\lambda, \mu))$, viewed as a free $\mathbb{Z}\left[q, q^{-1}\right]$ module. Composition bifunctors

$$
\dot{u}\left(\lambda, \lambda^{\prime}\right) \times \dot{u}\left(\lambda^{\prime}, \lambda^{\prime \prime}\right) \longrightarrow \dot{u}\left(\lambda, \lambda^{\prime \prime}\right)
$$

induce $\mathbb{Z}\left[q, q^{-1}\right]$-bilinear maps

$$
K_{0}\left(\dot{u}\left(\lambda, \lambda^{\prime}\right)\right) \otimes K_{0}\left(\dot{u}\left(\lambda^{\prime}, \lambda^{\prime \prime}\right)\right) \longrightarrow K_{0}\left(\dot{u}\left(\lambda, \lambda^{\prime \prime}\right)\right)
$$


turning $K_{0}(\dot{U})$ into a $\mathbb{Z}\left[q, q^{-1}\right]$-linear additive category with objects $\lambda \in X$. Alternatively, we may view $K_{0}(\dot{\mathcal{U}})$ as a non-unital $\mathbb{Z}\left[q, q^{-1}\right]$-algebra

$$
\bigoplus_{\lambda, \mu \in X} K_{0}(u)(\lambda, \mu)
$$

with a family of idempotents $\left[\mathbf{1}_{\lambda}\right]$. The set $\dot{\mathcal{B}}:=\bigoplus_{\lambda, \mu \in X} \dot{\mathcal{B}}(\lambda, \mu)$ gives rise to a basis $[\dot{\mathcal{B}}]:=\{[b]\}_{b \in \dot{\mathcal{B}}}$ of idempotented $\mathbb{Z}\left[q, q^{-1}\right]$-algebra $K_{0}(\dot{\mathcal{U}})$. Notice that basis elements are defined up to multiplication by powers of $q$; we will not try to choose a canonical grading normalization here. Multiplication in this basis has coefficients in $\mathbb{N}\left[q, q^{-1}\right]$.

Both ${ }_{\mathcal{A}} \dot{\mathbf{U}}$ and $K_{0}(\dot{\mathcal{U}})$ are idempotented $\mathbb{Z}\left[q, q^{-1}\right]$-algebras, with the idempotents $1_{\lambda}$ and $\left[\mathbf{1}_{\lambda}\right]$ labelled by $\lambda \in X$. To relate the two algebras, send $1_{\lambda}$ to $\left[\mathbf{1}_{\lambda}\right]$ and, more generally, $E_{\boldsymbol{i}} 1_{\lambda}$ to $\left[\mathcal{E}_{i} \mathbf{1}_{\lambda}\right]$ for all $\boldsymbol{i} \in \mathrm{SSeqd}$.

Proposition 3.27. The assignment $E_{\boldsymbol{i}} 1_{\lambda} \longrightarrow\left[\mathcal{E}_{i} \mathbf{1}_{\lambda}\right]$ extends to a $\mathbb{Z}\left[q, q^{-1}\right]$-algebra homomorphism

$$
\gamma:{ }_{\mathcal{A}} \dot{\mathbf{U}} \longrightarrow K_{0}(\dot{u}) \text {. }
$$

Multiplication by $q$ corresponds to the grading shift $\{1\}$.

Proof. $K_{0}(\dot{U})$ is a free $\mathbb{Z}\left[q, q^{-1}\right]$-module, so it is enough to check that the assignment above extends to a homomorphism of $\mathbb{Q}(q)$-algebras

$$
\gamma_{\mathbb{Q}(q)}: \dot{\mathbf{U}} \rightarrow K_{0}(\dot{U}) \otimes_{\mathbb{Z}\left[q, q^{-1}\right]} \mathbb{Q}(q)
$$

${ }_{(}{ }_{\mathcal{H}} \dot{\mathbf{U}}$ is also a free $\mathbb{Z}\left[q, q^{-1}\right]$-module, but this fact is not needed in the proof). Propositions $3.24,3.25$, and 3.26 show that defining relations of $\dot{\mathbf{U}}$ lift to 2 -isomorphisms of 1-morphisms in $\dot{U}$ and, therefore, descend to relations in the Grothendieck group $K_{0}(\dot{\mathcal{U}})$. Restricting $\gamma_{\mathbb{Q}(q)}$ to ${ }_{\mathcal{A}} \dot{\mathbf{U}}$ gives a homomorphism of $\mathbb{Z}\left[q, q^{-1}\right]$-algebras with the image of the homomorphism lying in $K_{0}(\dot{U})$.

For each $\lambda, \mu \in X$, the homomorphism $\gamma$ restricts to a homomorphism of $\mathbb{Z}\left[q, q^{-1}\right]$-modules

$$
1_{\mu}\left({ }_{\mathcal{H}} \dot{\mathbf{U}}\right) 1_{\lambda} \longrightarrow K_{0}(\dot{U}(\lambda, \mu)) .
$$

Proposition 3.28. The homomorphism $\gamma$ intertwines (anti)automorphisms $\psi, \omega, \sigma$, $\tau$ of ${ }_{\mathcal{A}} \dot{\mathbf{U}}$ with (anti)automorphisms $[\tilde{\psi}],[\tilde{\omega}]$, [ $\left.\tilde{\sigma}\right]$, and $[\tilde{\tau}]$ of $K_{0}(\dot{\mathcal{U}})$, respectively, i.e., the following diagrams commute:
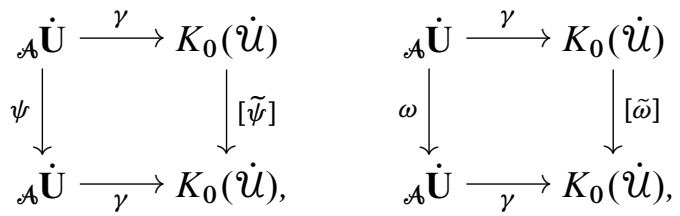

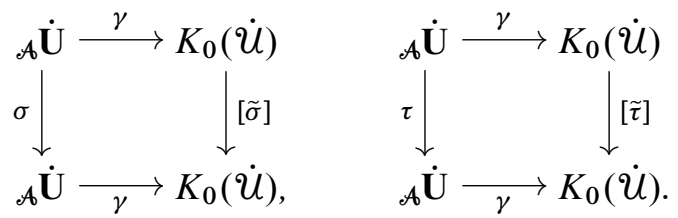

$[\widetilde{\psi}]$ denotes the induced action of $\widetilde{\psi}$ on the Grothendieck group, etc.

Proof. The proof follows from definitions and our construction of $\gamma$.

The 2-isomorphisms $\tilde{\omega}, \tilde{\sigma}, \tilde{\psi}$ on $U^{*}$ give isomorphisms of graded $\mathbb{k}$-vector spaces

$$
\begin{aligned}
& U^{*}(x, y) \cong U^{*}(\tilde{\omega}(x), \tilde{\omega}(y)), \\
& u^{*}(x, y) \cong\left(U^{*}\right)^{\mathrm{op}}(\tilde{\sigma}(x), \tilde{\sigma}(y))=U^{*}(\tilde{\sigma}(x), \tilde{\sigma}(y)), \\
& u^{*}(x, y) \cong\left(u^{*}\right)^{\mathrm{co}}(\tilde{\psi}(x), \tilde{\psi}(y))=U^{*}(\tilde{\psi}(y), \tilde{\psi}(x)) .
\end{aligned}
$$

On Grothendieck rings these isomorphisms give equalities

$$
\begin{aligned}
& \langle x, y\rangle=\langle\omega(x), \omega(y)\rangle, \\
& \langle x, y\rangle=\langle\sigma(x), \sigma(y)\rangle, \\
& \langle x, y\rangle=\langle\psi(y), \psi(x)\rangle,
\end{aligned}
$$

which should be compared with Propositions 26.1.4 and 26.1.6 in [24] and property (v) of the semilinear form. The last equality expressed in terms of the bilinear form $($, ), with $x$ replaced by $\psi(x)$, gives the identity $(x, y)=(y, x)$.

3.7. Idempotented rings. An idempotented $\operatorname{ring} A$ is an associative ring, not necessarily unital, equipped with a system of idempotents $\left\{1_{x}\right\}$, over elements $x$ of some set $Z$. We require orthogonality $1_{x} 1_{y}=\delta_{x, y} 1_{x}$ and decomposition

$$
A=\bigoplus_{x, y \in Z} 1_{y} A 1_{x}
$$

By a (left) $A$-module we mean an $A$-module $M$ such that

$$
M=\bigoplus_{x \in Z} 1_{x} M
$$

In this paper three collections of idempotented rings appear:

- Lusztig's $\dot{\mathbf{U}}$ and its integral form ${ }_{\mathcal{A}} \dot{\mathbf{U}}$. Here $Z=X$, the weight lattice,

$$
\dot{\mathbf{U}}=\bigoplus_{\lambda, \mu \in X} 1_{\mu} \dot{\mathbf{U}} 1_{\lambda}, \quad{ }_{\mathcal{A}} \dot{\mathbf{U}}=\bigoplus_{\lambda, \mu \in X} 1_{\mu}\left({ }_{\mathcal{A}} \dot{\mathbf{U}}\right) 1_{\lambda} .
$$


- The Grothendieck groups $K_{0}\left(\operatorname{Kar}\left(\mathcal{U}^{*}\right)\right)$ and $K_{0}(\dot{\mathcal{U}})$, the latter defined in Section 3.6. Again, the parameterizing set $Z=X$. We only study

$$
K_{0}(\dot{u})=\bigoplus_{\lambda, \mu \in X}\left[\mathbf{1}_{\mu}\right] K_{0}(\dot{U})\left[\mathbf{1}_{\lambda}\right]
$$

with $\left\{\left[\mathbf{1}_{\lambda}\right]\right\}_{\lambda \in X}$ being the system of idempotents in $K_{0}(\dot{U})$. The map

$$
\gamma:{ }_{A} \dot{\mathbf{U}} \rightarrow K_{0}(\dot{u})
$$

is a homomorphism of idempotented rings.

- For each $\lambda, \mu \in X$ the $\mathbb{Z}$-graded ring

$$
{ }_{\mu} u_{\lambda}^{*}:=\bigoplus_{\boldsymbol{i}, \boldsymbol{j}} \operatorname{HOM}_{u}\left(\mathcal{E}_{\boldsymbol{i}} \mathbf{1}_{\lambda}, \mathcal{E}_{\boldsymbol{j}} \mathbf{1}_{\lambda}\right)
$$

where the sum is over all $\boldsymbol{i}, \boldsymbol{j} \in \mathrm{SSeq}$ with $\boldsymbol{i}_{X}, \boldsymbol{j}_{X}=\mu-\lambda$. Thus, the sum is over all sequences such that $E_{\boldsymbol{i}} 1_{\lambda}, E_{\boldsymbol{j}} 1_{\lambda}$ have left weight $\mu$. The parameterizing set $Z=\left\{i \in \mathrm{SSeq} \mid \boldsymbol{i}_{X}=\lambda-\mu\right\}$.

The category $\dot{U}(\lambda, \mu)$ is equivalent to the category of right finitely generated graded projective ${ }_{\mu} U_{\lambda}^{*}$-modules and grading preserving homomorphisms. The equivalence functor

$$
\dot{U}(\lambda, \mu) \rightarrow \text { pmod- } \mu \mathcal{U}_{\lambda}^{*}
$$

takes $\varepsilon_{\boldsymbol{i}} \mathbf{1}_{\lambda}$ to

$$
{ }_{i, \lambda} P:=\bigoplus_{j \in Z} \operatorname{HOM}_{u}\left(\varepsilon_{\boldsymbol{i}} \mathbf{1}_{\lambda}, \mathcal{E}_{\boldsymbol{j}} \mathbf{1}_{\lambda}\right)
$$

and, more generally, an object $\left(\varepsilon_{\boldsymbol{i}} \mathbf{1}_{\lambda}, e\right)$ to

$$
{ }_{i, \lambda, e} P:=\bigoplus_{j \in Z} \operatorname{HOM}_{\dot{u}}\left(\left(\varepsilon_{\boldsymbol{i}} \mathbf{1}_{\lambda}, e\right), \mathcal{E}_{\boldsymbol{j}} \mathbf{1}_{\lambda}\right)
$$

The Grothendieck group $K_{0}(\dot{U}(\lambda, \mu))$ is isomorphic to the Grothendieck group of pmod- $\mu U_{\lambda}^{*}$.

Notice that we get idempotented rings from the 2-category $\dot{U}$ in various ways:

1) as the Grothendieck ring/pre-additive category $K_{0}(\dot{u})$ of $\dot{u}$,

2) as rings associated to categories $\dot{U}(\lambda, \mu)$.

The 2-category $\dot{U}$ can itself be viewed as an idempotented monoidal category. 
We encode these observations into a diagram.

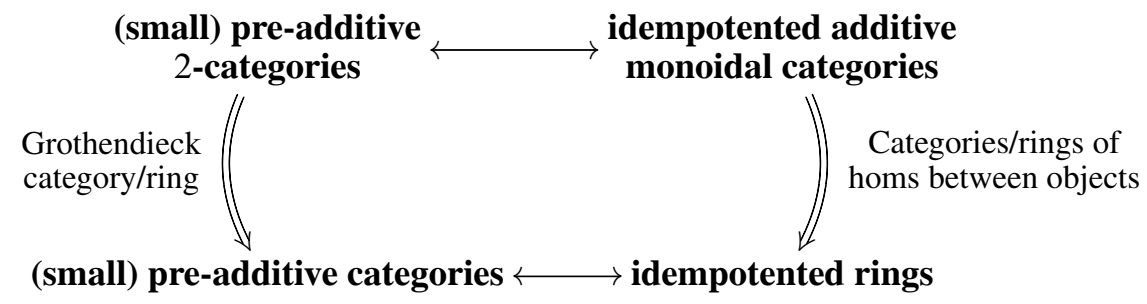

3.8. Surjectivity of $\boldsymbol{\gamma}$. To prove surjectivity of $\gamma$ we will analyze the diagram of $K$-groups and their homomorphisms induced by the diagram (3.24) and trace minimal idempotents there, but first recall some basics of Grothendieck groups of finitedimensional algebras (as a model example) and graded algebras.

3.8.1. $K_{\mathbf{0}}$ of finite-dimensional algebras. A homomorphism of rings $\alpha: A \rightarrow B$ induces a homomorphism of $K_{0}$-groups

$$
K_{0}(\alpha): K_{0}(A) \rightarrow K_{0}(B)
$$

of finitely generated projective modules. For definition and properties of $K_{0}$ we refer the reader to [28], [32, Chapter II].

Assume that $A$ and $B$ are finite-dimensional $\mathbb{k}$-algebras, for a field $\mathbb{k}$, and $\alpha$ is a $\mathbb{k}$-algebra homomorphism. If $\alpha$ is surjective then $K_{0}(\alpha)$ is surjective as well. On the level of idempotents, if $1=e_{1}+\cdots+e_{k}$ is a decomposition of $1 \in A$ into a sum of mutually orthogonal minimal idempotents, then $A e_{s}$ is an indecomposable projective $A$-module, $K_{0}(A)$ is a free abelian group with a basis $\left\{\left[A e_{r}\right]\right\}_{r \in S}$, for a subset $S \subset\{1, \ldots, k\}$. $S$ is any maximal subset with the property that $A e_{s} \not A e_{t}$ as left $A$-modules for any $s, t \in S, s \neq t$. Applying $\alpha$ to the above decomposition results in the equation

$$
B \ni 1=\alpha\left(e_{1}\right)+\cdots+\alpha\left(e_{k}\right) .
$$

where each $\alpha\left(e_{s}\right)$ is either 0 or a minimal idempotent in $B$ (the minimality of $\alpha\left(e_{s}\right)$ follows from the idempotent lifting for $\alpha$, by first reducing to the case of semisimple $B$ by quotienting out by the Jacobson radical of $B)$. Relabel minimal idempotents so that $\alpha\left(e_{1}\right), \ldots, \alpha\left(e_{m}\right) \neq 0, \alpha\left(e_{m+1}\right)=\cdots=\alpha\left(e_{k}\right)=0$ (elements of $S$ get permuted as well). Then $1=\alpha\left(e_{1}\right)+\cdots+\alpha\left(e_{m}\right)$ is a decomposition of $1 \in B$ into a sum of mutually orthogonal minimal idempotents, $B \alpha\left(e_{r}\right)$ is an indecomposable projective $B$-module, $1 \leq r \leq m$, and

$$
\left\{\left[B \alpha\left(e_{r}\right)\right]\right\}_{r \in S \cap\{1, \ldots, m\}}
$$

is a basis of $K_{0}(B)$. 
Remark 3.29. If $\mathrm{A}$ is a finite-dimensional $\mathbb{k}$-algebra, the quotient map $A \rightarrow A / J(A)$, where $J(A)$ is the Jacobson radical of $A$, induces an isomorphism of $K_{0}$-groups

$$
K_{0}(A) \cong K_{0}(A / J(A)) .
$$

Indeed, $J(A)$ is a nilpotent ideal, $J(A)^{N}=0$ for sufficiently large $N$, and the quotient by a nilpotent ideal induces an isomorphism of $K_{0}$ 's; see [32], Lemma 2.2 in Chapter II.

Proposition 3.30. If $A, B$ are finite-dimensional $\mathbb{k}$-algebras such that all simple Aand $B$-modules are absolutely irreducible over $\mathbb{k}$, then

$$
K_{0}(A) \otimes_{\mathbb{Z}} K_{0}(B) \cong K_{0}\left(A \otimes_{\mathbb{k}} B\right)
$$

via an isomorphism that takes $[P] \otimes[Q]$ for projective $A$, respectively $B$, modules $P$ and $Q$ to $\left[P \otimes_{\mathbb{k}} Q\right]$.

Proof. By passing to $A / J(A), B / J(B)$ and using the above remark, we reduce to the case of semisimple $A$ and $B$. Then both $A$ and $B$ are finite products of the field $\mathbb{k}$ and the proposition follows.

3.8.2. $K_{0}$ of graded algebras. From here on we only consider $\mathbb{Z}$-graded $\mathbb{k}$-algebras, for a field $\mathbb{k}$. For a $\mathbb{Z}$-graded $\mathbb{k}$-algebra $A=\oplus_{a \in \mathbb{Z}} A_{a}$ denote by $K_{0}(A)$ the Grothendieck group of finitely generated graded left projective $A$-modules. $K_{0}(A)$ is a $\mathbb{Z}\left[q, q^{-1}\right]$-module.

Throughout this subsection we assume that all weight spaces $A_{a}$ are finite-dimensional, and the grading is bounded below: $A_{a}=0$ for all $a \ll 0$.

Let $\operatorname{PI}(A)$ be the set of isomorphism classes of indecomposable graded projective $A$-modules, up to a grading shift. We can normalize the grading and choose one representative $Q$ for each element of $\operatorname{PI}(A)$ so that 0 is the lowest nontrivial degree of $Q$. We write $Q \in \operatorname{PI}(A)$.

Proposition 3.31. For $A$ as above, $K_{0}(A)$ is a free $\mathbb{Z}\left[q, q^{-1}\right]$-module with the basis $\{[Q]\}_{Q \in \operatorname{PI}(A)}$.

Proof. Since each weight space of $A$ is finite-dimensional, the Krull-Schmidt property holds for graded projective finitely generated $A$-modules. Any such module has a unique, up to isomorphism, decomposition as a direct sum of indecomposables, and $K_{0}(A)$ is a free abelian group with a basis labelled by isomorphism classes of indecomposable projectives. Boundedness of $A$ from below ensures that an indecomposable projective is not isomorphic to itself with a shifted grading, implying that $K_{0}(A)$ is a free $\mathbb{Z}\left[q, q^{-1}\right]$-module and the rest of the proposition.

We say that a 2 -sided homogeneous ideal $J$ of $A$ is virtually nilpotent if for any $a \in \mathbb{Z}$ the weight space $\left(J^{N}\right)_{a}=0$ for sufficiently large $N$. 
Proposition 3.32. For $A$ as above and $J$ a virtually nilpotent ideal of $A$ the quotient map $A \rightarrow A / J$ induces an isomorphism $K_{0}(A) \cong K_{0}(A / J)$.

Proof. Proposition follows from the lifting idempotents property. This is the graded version of Lemma 2.2 in [32, Chapter 2].

Proposition 3.33. Let $\alpha: A \rightarrow B$ be a surjective homomorphism of finite-dimensional graded $\mathbb{k}$-algebras. Then the induced map $K_{0}(\alpha)$ is surjective.

Proof. The argument is essentially the same as in the nongraded case discussed earlier. In the bases of $K_{0}(A)$ and $K_{0}(B)$ given by indecomposable projective modules, the map $K_{0}(\alpha)$ sends some basis elements of $K_{0}(A)$ to 0 and the rest go bijectively to the basis of $K_{0}(B)$ (possibly after grading shifts).

Finally, we discuss $K_{0}$ of graded idempotented algebras. Let $A$ be an associative graded $\mathbb{k}$-algebra, possibly nonunital, with a family of mutually orthogonal degree 0 idempotents $1_{x} \in A, x \in Z$, such that

$$
A=\bigoplus_{x, y \in Z} 1_{y} A 1_{x}
$$

(compare with the definition of idempotented ring in Section 3.7). We say that $A$ is a graded idempotented $\mathbb{k}$-algebra. By a graded finitely generated projective $A$ module we mean a homogeneous direct summand of a finite direct sum (with finite multiplicities) of graded left $A$-modules $A 1_{x}\{t\}$, over $x \in Z$ and $t \in \mathbb{Z}$. By $K_{0}(A)$ we denote the corresponding Grothendieck group, which is again a $\mathbb{Z}\left[q, q^{-1}\right]$-module.

We assume that for each $x, y \in Z$ the graded $\mathbb{k}$-vector space $1_{y} A 1_{x}$ is bounded below and has finite-dimensional weight spaces.

Proposition 3.34. For $A$ as above, $K_{0}(A)$ is a free $\mathbb{Z}\left[q, q^{-1}\right]$-module with a basis given by isomorphism classes of indecomposables, up to grading shifts.

Proof. The proof is essentially the same as that of Proposition 3.31. The difference is in the absence of a canonical grading normalization for a representative $Q$ of an isomorphism class of indecomposables up to grading shifts. This normalization can be chosen ad hoc, of course.

3.8.3. A triangle of $\boldsymbol{K}_{\mathbf{0}}$ 's. We will work in the graded case, so that the rings are $\mathbb{Z}$ graded and $K_{0}$ are $\mathbb{Z}\left[q, q^{-1}\right]$-modules. Consider the diagram of $\mathbb{Z}\left[q, q^{-1}\right]$-modules

$$
\begin{aligned}
& K_{0}\left(R(v) \otimes R\left(v^{\prime}\right) \otimes \Pi_{\lambda}\right) \\
& \left.K_{0}\left(\operatorname{END}_{u} \underset{K_{0}(\alpha) \downarrow}{\downarrow} \mathcal{E}_{v,-v^{\prime}} \mathbf{1}_{\lambda}\right)\right) \underset{K_{0}(\alpha)}{K_{0}(\beta \alpha)} K_{0}\left(R_{\nu,-v^{\prime}, \lambda}\right)
\end{aligned}
$$


given by applying the $K_{0}$ functor to the commutative triangle in (3.24).

Recall that in [16], [17] we constructed an isomorphism of $\mathbb{Z}\left[q, q^{-1}\right]$-modules

$$
K_{0}(R(v)) \cong{ }_{\mathcal{A}} \mathbf{f}_{v},
$$

where ${ }_{\mathcal{A}} \mathbf{f}_{v}$ is the weight $v$ summand of the $\mathbb{Z}\left[q, q^{-1}\right]$-algebra ${ }_{\mathcal{A}} \mathbf{f}$. Likewise,

$$
K_{0}\left(R\left(v^{\prime}\right)\right) \cong{ }_{A} \mathbf{f}_{v^{\prime}} .
$$

$R(v)$, respectively $R\left(v^{\prime}\right)$, is a free finite rank graded module over its center $Z(R(v)) \cong \operatorname{Sym}(v)$, respectively $\operatorname{Sym}\left(v^{\prime}\right)$, isomorphic to the $\mathbb{k}$-algebra of polynomials in several homogeneous generators, all of positive degree. Hence, $R(v) \otimes$ $R\left(v^{\prime}\right) \otimes \Pi_{\lambda}$ is a free finite rank graded module over the central graded polynomial algebra $\operatorname{Sym}(v) \otimes \operatorname{Sym}\left(v^{\prime}\right) \otimes \Pi_{\lambda}$. This algebra contains a homogeneous augmentation ideal $\mathrm{Sym}^{+}$of codimension 1. Let

$$
J=\left(R(v) \otimes R\left(v^{\prime}\right) \otimes \Pi_{\lambda}\right) \operatorname{Sym}^{+}
$$

be the corresponding 2-sided ideal of $R(v) \otimes R\left(v^{\prime}\right) \otimes \Pi_{\lambda}$, and consider the quotient algebra

$$
\begin{aligned}
R & :=R(v) \otimes R\left(v^{\prime}\right) \otimes \Pi_{\lambda} / J \\
& \cong\left(R(v) /\left(R(v) \cdot \operatorname{Sym}^{+}(v)\right)\right) \otimes\left(R\left(v^{\prime}\right) /\left(R\left(v^{\prime}\right) \cdot \operatorname{Sym}^{+}\left(v^{\prime}\right)\right)\right) .
\end{aligned}
$$

$R$ is a finite-dimensional $\mathbb{k}$-algebra, and the quotient map

$$
\alpha^{\prime}: R(v) \otimes R\left(v^{\prime}\right) \otimes \Pi_{\lambda} \longrightarrow R
$$

induces an isomorphism of $K_{0}$-groups

$$
K_{0}\left(\alpha^{\prime}\right): K_{0}\left(R(v) \otimes R\left(v^{\prime}\right) \otimes \Pi_{\lambda}\right) \longrightarrow K_{0}(R),
$$

since the ideal $J$ is virtually nilpotent, see Proposition 3.32 .

We proved in [16], [17] that any simple graded $R(v)$-module is absolutely irreducible for any field $\mathbb{k}$, same for simple graded $R(v) /\left(R(v) \mathrm{Sym}^{+}\right)$-modules. Also note that $K_{0}\left(\Pi_{\lambda}\right) \cong \mathbb{Z}\left[q, q^{-1}\right]$, since $\Pi_{\lambda}$ is a graded local ring. The chain of isomorphisms

$$
K_{0}\left(R(v) \otimes R\left(v^{\prime}\right) \otimes \Pi_{\lambda}\right) \cong K_{0}(R) \cong K_{0}(R(v)) \otimes K_{0}\left(R\left(v^{\prime}\right)\right) \cong{ }_{\mathcal{A}} \mathbf{f}_{v} \otimes{ }_{\mathcal{A}} \mathbf{f}_{v^{\prime}}
$$

establishes the following result:

Proposition 3.35. There is a canonical isomorphism

$$
K_{0}\left(R(v) \otimes R\left(v^{\prime}\right) \otimes \Pi_{\lambda}\right) \cong{ }_{\mathcal{A}} \mathbf{f}_{v} \otimes{ }_{\mathcal{A}} \mathbf{f}_{v^{\prime}}
$$

induced by isomorphisms $K_{0}(R(v)) \cong{ }_{\mathcal{A}} \mathbf{f}_{v}$ and $K_{0}\left(R\left(v^{\prime}\right)\right) \cong{ }_{\mathcal{A}} \mathbf{f}_{v^{\prime}}$ constructed in [16], [17]. 
This proposition gives us a grip on the top term in the diagram (3.26).

Proposition 3.36. $K_{0}(\beta \alpha)$ is surjective.

Proof. Since $\beta \alpha$ is surjective, $\beta \alpha(J)$ is a 2 -sided ideal of $R_{v,-v^{\prime}, \lambda}$. Start with a commutative square of surjective algebra homomorphisms

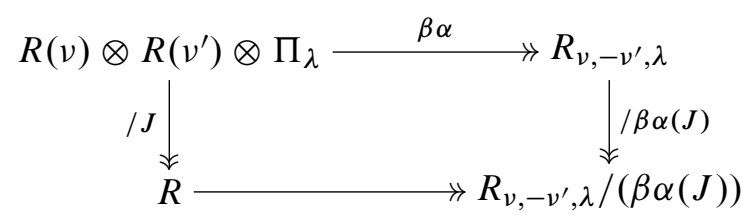

and apply functor $K_{0}$ to obtain a commutative diagram

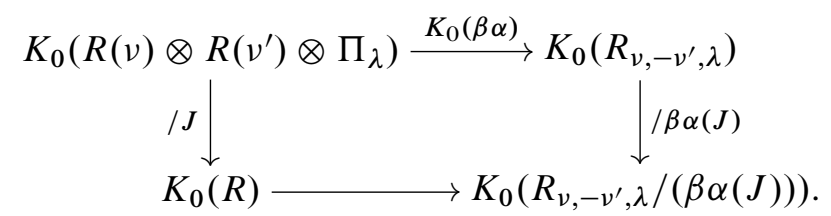

The vertical arrows are isomorphisms since $J$ and $\beta \alpha(J)$ are virtually nilpotent ideals. The bottom arrow is surjective, by Proposition 3.33, since $R$ and $R_{v,-v^{\prime}, \lambda}$ are finite-dimensional over $\mathbb{k}$. Surjectivity of the top arrow follows.

Corollary 3.37. $K_{0}(\beta)$ is surjective.

These observations are summarized in the following enhancement of (3.26).

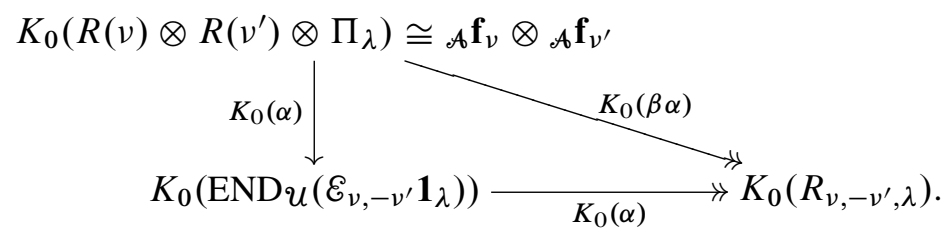

3.8.4. Idempotents in $\dot{\boldsymbol{u}}$. Let $1=e_{1}+\cdots+e_{k}$ and $1=e_{1}^{\prime}+\cdots+e_{k^{\prime}}^{\prime}$ be a decomposition of $1 \in \operatorname{End}_{\dot{u}}\left(\varepsilon_{v} \mathbf{1}_{\lambda}\right) \cong R(v)_{0}$ and of $1 \in \operatorname{End}_{\dot{u}}\left(\mathcal{E}_{-v^{\prime}} \mathbf{1}_{\lambda}\right) \cong R\left(v^{\prime}\right)_{0}$, respectively, into the sum of minimal mutually orthogonal idempotents. Here $R(v)_{0}$ denotes the degree 0 subalgebra of $R(v)$. Each term in the decomposition

$$
1=\sum_{r=1}^{k} \sum_{r^{\prime}=1}^{k^{\prime}} e_{r} \otimes e_{r^{\prime}}^{\prime} \otimes 1
$$

of $1 \in R(v) \otimes R\left(v^{\prime}\right) \otimes \Pi_{\lambda}$ is a minimal degree 0 idempotent in view of the discussion preceding Proposition 3.35. 
Let

$$
e_{r, r^{\prime}}:=\alpha\left(e_{r} \otimes e_{r^{\prime}}^{\prime} \otimes 1\right) \in \operatorname{End}_{\dot{u}}\left(\varepsilon_{v,-v^{\prime}} \mathbf{1}_{\lambda}\right)
$$

be the corresponding idempotent in the endomorphism algebra of $\varepsilon_{\nu,-v^{\prime}} \mathbf{1}_{\lambda}$ which may not be minimal. Then

$$
e_{r, r^{\prime}}=\sum_{r^{\prime \prime}=1}^{k\left(r, r^{\prime}\right)} e_{r, r^{\prime}, r^{\prime \prime}}
$$

can be decomposed into a sum of minimal mutually orthogonal degree zero idempotents $e_{r, r^{\prime}, r^{\prime \prime}} \in \operatorname{End}_{\dot{u}}\left(\mathcal{E}_{\nu,-v^{\prime}} \mathbf{1}_{\lambda}\right)$.

The homomorphism $\beta \alpha$ induces a surjection of Grothendieck groups and maps each minimal idempotent $e_{r} \otimes e_{r^{\prime}}^{\prime} \otimes 1$ either to 0 or to a minimal degree 0 idempotent in $R_{v,-v^{\prime}, \lambda}$ (Proposition 3.36). Consequently, for each $\left(r, r^{\prime}, r^{\prime \prime}\right)$ the image $\beta\left(e_{r, r^{\prime}, r^{\prime \prime}}\right)$ is either 0 or a minimal idempotent in $R_{v,-v^{\prime}, \lambda}$. Moreover, for each $\left(r, r^{\prime}\right)$ at most one of $\beta\left(e_{r, r^{\prime}, r^{\prime \prime}}\right) \neq 0$ in $R_{v,-v^{\prime}, \lambda}$. We can relabel idempotents so that $\beta\left(e_{r, r^{\prime}, 1}\right) \neq 0$ and $\beta\left(e_{r, r^{\prime}, r^{\prime \prime}}\right)=0$ for $r^{\prime \prime}>1$ whenever $\beta \alpha\left(e_{r} \otimes e_{r^{\prime}}^{\prime} \otimes 1\right) \neq 0$. Necessarily, $\beta\left(e_{r, r^{\prime}, r^{\prime \prime}}\right)=0$ for all $r^{\prime \prime}$ if $\beta \alpha\left(e_{r} \otimes e_{r^{\prime}}^{\prime} \otimes 1\right)=0$.

If $\beta\left(e_{r} \otimes e_{r^{\prime}}^{\prime} \otimes 1\right)=0$ then $e_{r, r^{\prime}, r^{\prime \prime}} \in \mathcal{I}_{v,-v^{\prime}, \lambda}$. A homogeneous element $a \in \mathcal{I}_{v,-v^{\prime}, \lambda}$ can be written as a finite sum $a=\sum_{s=1}^{u} a_{s}^{\prime} a_{s}$, where $a_{s}, a_{s}^{\prime}$ are homogeneous,

$$
a_{s} \in U^{*}\left(\mathcal{E}_{\nu,-v} \mathbf{1}_{\lambda}, \mathcal{E}_{\boldsymbol{i}(s)} \mathbf{1}_{\lambda}\right), \quad a_{s}^{\prime} \in \mathcal{U}^{*}\left(\mathcal{E}_{\boldsymbol{i}(s)} \mathbf{1}_{\lambda}, \mathcal{E}_{\nu,-v} \mathbf{1}_{\lambda}\right),
$$

and $\boldsymbol{i}(\boldsymbol{s}) \in$ SSeq with $\|\boldsymbol{i}(\boldsymbol{s})\|<\|v\|+\left\|v^{\prime}\right\|$. Indeed, an element of $\mathcal{I}_{v,-v^{\prime}, \lambda}$ can be written as a linear combination of diagrams with U-turns. Cutting each diagram in the middle allows us to view it as composition

$$
\varepsilon_{\nu,-v^{\prime}} \mathbf{1}_{\lambda} \rightarrow \varepsilon_{i(s)} \mathbf{1}_{\lambda} \rightarrow \varepsilon_{\nu,-v^{\prime}} \mathbf{1}_{\lambda}
$$

with the length $\|\boldsymbol{i}(s)\|$ of the sequence $i(s)$ strictly less than the sum of lengths $\|v\|+\left\|v^{\prime}\right\|$.

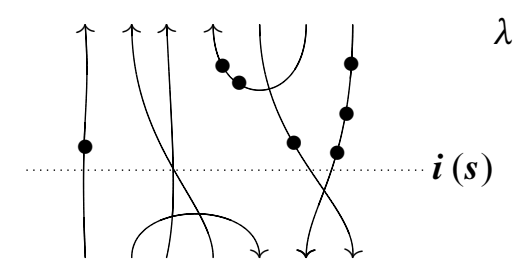

Choose such a decomposition for each

$$
e_{r, r^{\prime}, r^{\prime \prime}} \in \mathcal{I}_{v,-v^{\prime}, \lambda}, \quad e_{r, r^{\prime}, r^{\prime \prime}}=\sum_{s=1}^{u} a_{s}^{\prime} a_{s}
$$

where in the notations we suppress dependence of $u\left(r, r^{\prime}, r^{\prime \prime}\right), a_{s}\left(r, r^{\prime}, r^{\prime \prime}\right)$ and $a_{s}^{\prime}\left(r, r^{\prime}, r^{\prime \prime}\right)$ on the three parameters. Multiplication by $e_{r, r^{\prime}, r^{\prime \prime}}$ is the identity endomorphism of $\left(\varepsilon_{v,-v^{\prime}} \mathbf{1}_{\lambda}, e_{r, r^{\prime}, r^{\prime \prime}}\right)$. We can view this indecomposable 1-morphism 
of $\dot{U}$ as an indecomposable projective module (call it $P$ ) over the graded idempotented ring $\mu U_{\lambda}^{*}, \mu=\lambda+v_{X}-v_{X}^{\prime}$. Then the identity endomorphism of $P$ factors through projective module $Q$ corresponding to the direct sum $\bigoplus_{s=1}^{u} \mathcal{E}_{i(s)} \mathbf{1}_{\lambda}\left\{t_{s}\right\}$

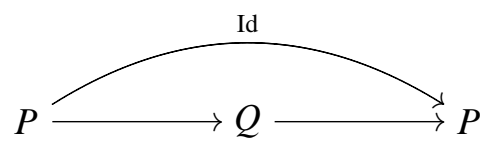

for some $t_{s} \in \mathbb{Z}$. Therefore, $P$ is isomorphic to a direct summand of $Q$, and the 1-morphism $\left(\varepsilon_{v,-v^{\prime}} \mathbf{1}_{\lambda}, e_{r, r^{\prime}, r^{\prime \prime}}\right)$ of $\dot{U}$ is isomorphic to a direct summand of $\bigoplus_{s=1}^{u} \varepsilon_{i(s)} \mathbf{1}_{\lambda}\left\{t_{s}\right\}$.

Define the width $\|P\|$ of an indecomposable 1-morphism $P \in \mathrm{Ob}(\dot{U}(\lambda, \mu))$ as the smallest $m$ such that $P$ is isomorphic to a direct summand of $\mathcal{E}_{i} \mathbf{1}_{\lambda}\{t\}$ for some $\boldsymbol{i} \in \mathrm{SSeq},\|\boldsymbol{i}\|=m$ and $t \in \mathbb{Z}$.

For example, if $P$ has width 0 , then $P$ is isomorphic to a direct summand of $\mathbf{1}_{\lambda}\{t\}$ for some $\lambda$ and $t$. The 1-morphism $\mathbf{1}_{\lambda}\{t\}$ is indecomposable, since its endomorphism ring $\dot{U}\left(\mathbf{1}_{\lambda}\{t\}, \mathbf{1}_{\lambda}\{t\}\right)=\mathbb{k}$, or 0 (a possibility if the calculus is degenerate). This implies that any width zero 1 -morphism is isomorphic to $\mathbf{1}_{\lambda}\{t\}$.

Lemma 3.38. If $P$ has width $m$ then $P$ is isomorphic to a direct summand of $\mathcal{E}_{v,-v^{\prime}} \mathbf{1}_{\lambda}\{t\}$ for some $v, v^{\prime} \in \mathbb{N}[I],\|v\|+\left\|v^{\prime}\right\|=m, \lambda \in X$, and $t \in \mathbb{Z}$.

Proof. If $i, j \in I$ and $\boldsymbol{i}=\boldsymbol{i}^{\prime}-i+j \boldsymbol{i}^{\prime \prime}$ has length $m$, then $P$ is direct summand of $\mathcal{E}_{i} \mathbf{1}_{\lambda}\{t\}$ if and only if it is a direct summand of $\mathcal{E}_{\boldsymbol{i}^{\prime}+j-i \boldsymbol{i}^{\prime \prime}} \mathbf{1}_{\lambda}\{t\}$. Indeed, these two 1morphisms are either isomorphic (if $i \neq j$ ) or differ by direct summands $\mathcal{E}_{\boldsymbol{i}^{\prime} \boldsymbol{i}^{\prime \prime}} \mathbf{1}_{\lambda}\left\{t^{\prime}\right\}$, all whose indecomposable summands have width at most $m-2$, and thus cannot be isomorphic to $P$. By assumption, $P$ is isomorphic to a direct summand of $\mathcal{E}_{\boldsymbol{i}} \mathbf{1}_{\lambda}\{t\}$ with $\|\boldsymbol{i}\|=m$. Moving all positive terms of $\boldsymbol{i}$ to the left of all negative terms produces a sequence $\boldsymbol{j}\left(-\boldsymbol{j}^{\prime}\right)$ with $\boldsymbol{j}, \boldsymbol{j}^{\prime}$ positive, $\|\boldsymbol{j}\|+\left\|\boldsymbol{j}^{\prime}\right\|=m$ and $P$ being a summand of $\mathcal{E}_{\boldsymbol{j}(-\boldsymbol{j})} \mathbf{1}_{\lambda}\{t\}$. But this 1-morphism is a direct summand of $\mathcal{E}_{v,-v^{\prime}} \mathbf{1}_{\lambda}\{t\}$ with $v$, respectively $v^{\prime}$, being the weight of $\boldsymbol{j}$ and $\boldsymbol{j}^{\prime}$, respectively.

Proof of Theorem 1.1. We show that $[P]$ is in the image of $\gamma:{ }_{A} \dot{\mathbf{U}} \rightarrow K_{0}(\dot{U})$ by induction on the length of $P$. Let $P$ have length $m$. Then $P$ is a direct summand of $\varepsilon_{v,-v^{\prime}} \mathbf{1}_{\lambda}\{t\}$ for $v, v^{\prime}$ as above. By shifting the degree of $P$ down by $t$, $P \cong\left(\mathcal{E}_{v,-v^{\prime}} \mathbf{1}_{\lambda}, e_{r, r^{\prime}, r^{\prime \prime}}\right)$ for at least one minimal idempotent $e_{r, r^{\prime}, r^{\prime \prime}}$. We must have $r^{\prime \prime}=1$ and $\beta\left(e_{r, r^{\prime}, r^{\prime \prime}}\right) \neq 0$, for otherwise $P$ is isomorphic to a direct summand of $\bigoplus_{s} \mathcal{E}_{\boldsymbol{i}(s)} \mathbf{1}_{\lambda}\left\{t_{s}\right\}$, and since $\|\boldsymbol{i}(s)\|=m-2$, the width of $P$ is at most $m-2$, a contradiction. Thus $r^{\prime \prime}=1$ and $\beta\left(e_{r, r^{\prime}, r^{\prime \prime}}\right) \neq 0$.

From (3.27) We have

$$
[P]=\left[\mathcal{E}_{v,-v^{\prime}} \mathbf{1}_{\lambda}, e_{r, r^{\prime}, 1}\right]
$$


and

$$
\sum_{r^{\prime \prime}=1}^{k\left(r, r^{\prime}\right)}\left[\mathcal{E}_{v,-v^{\prime}} \mathbf{1}_{\lambda}, e_{r, r^{\prime}, r^{\prime \prime}}\right]=\left[\mathcal{E}_{v,-v^{\prime}} \mathbf{1}_{\lambda}, e_{r, r^{\prime}}\right]
$$

For $r^{\prime \prime}>1$ we have $\beta\left(e_{r, r^{\prime}, r^{\prime \prime}}\right)=0$, and $\left(\varepsilon_{v,-v^{\prime}} \mathbf{1}_{\lambda}, e_{r, r^{\prime}, r^{\prime \prime}}\right)$ is isomorphic to a direct summand of a finite sum of $\mathcal{E}_{\boldsymbol{i}} \mathbf{1}_{\lambda}\{t\}$, for sequences $\boldsymbol{i}$ of length $m-2$. Each indecomposable summand of $\left(\mathcal{E}_{\nu,-v^{\prime}} \mathbf{1}_{\lambda}, e_{r, r^{\prime}, r^{\prime \prime}}\right)$ has length at most $m-2$. By induction hypothesis, $\left[\mathcal{E}_{\nu,-v^{\prime}} \mathbf{1}_{\lambda}, e_{r, r^{\prime}, r^{\prime \prime}}\right]$ belongs to the image of $\gamma$ for all $2 \leq r^{\prime \prime} \leq k\left(r, r^{\prime}\right)$. Thus,

$$
\begin{aligned}
{[P]=\left[\mathcal{E}_{\nu,-v^{\prime}} \mathbf{1}_{\lambda}, e_{r, r^{\prime}, 1}\right] } & =\left[\mathcal{E}_{\nu,-v^{\prime}} \mathbf{1}_{\lambda}, e_{r, r^{\prime}}\right]-\sum_{r^{\prime \prime}=2}^{k\left(r, r^{\prime}\right)}\left[\mathcal{E}_{\nu,-v^{\prime}} \mathbf{1}_{\lambda}, e_{r, r^{\prime}, r^{\prime \prime}}\right] \\
& \in\left[\mathcal{E}_{\nu,-v^{\prime}} \mathbf{1}_{\lambda}, e_{r, r^{\prime}}\right]+\gamma\left({ }_{A} \dot{\mathbf{U}}\right) .
\end{aligned}
$$

It now suffices to show that $\left[\mathcal{E}_{\nu,-v^{\prime}} \mathbf{1}_{\lambda}, e_{r, r^{\prime}}\right]$ belongs to image of $\gamma$. But the idempotent $e_{r, r^{\prime}}$ is the image of $e_{r} \otimes e_{r^{\prime}} \otimes 1$ in $R(\nu) \otimes R\left(\nu^{\prime}\right) \otimes \Pi_{\lambda}$, and the Grothendieck group of the latter is isomorphic to ${ }_{\mathcal{A}} \mathbf{f}(v) \otimes{ }_{\mathcal{A}} \mathbf{f}\left(v^{\prime}\right)$. Therefore, $\left[\mathcal{E}_{v,-v^{\prime}}, e_{r, r^{\prime}}\right]$ is in the image of ${ }_{\mathcal{A}} \mathbf{f}(v) \otimes \mathcal{A}_{\mathcal{A}} \mathbf{f}\left(v^{\prime}\right)$ under the composition map

$$
\begin{aligned}
{ }_{\mathcal{A}} \mathbf{f}(v) \otimes{ }_{\mathcal{A}} \mathbf{f}\left(v^{\prime}\right) & \longrightarrow 1_{\mu}\left({ }_{\mathcal{A}} \dot{\mathbf{U}}\right) 1_{\lambda} \stackrel{\gamma}{\longrightarrow} K_{0}(\dot{U}(\lambda, \mu)), \\
x \otimes y & \longrightarrow x^{+} y^{-} 1_{\lambda}, \\
x \mapsto x^{+}:{ }_{\mathcal{A}} \mathbf{f}(v) & \rightarrow \mathbf{U}^{+}, \quad y \mapsto y^{-}:{ }_{\mathcal{A}} \mathbf{f}\left(v^{\prime}\right) \rightarrow \mathbf{U}^{-} .
\end{aligned}
$$

This completes the proof of surjectivity of $\gamma$.

3.9. Injectivity of $\boldsymbol{\gamma}$ in the non-degenerate case. Assume that our graphical calculus is non-degenerate for a given root datum and field $\mathbb{k}$, so that $B_{\boldsymbol{i}, \boldsymbol{j}, \lambda}$ is a basis of $\operatorname{HOM}_{u}\left(\varepsilon_{\boldsymbol{i}} \mathbf{1}_{\lambda}, \mathcal{E}_{\boldsymbol{j}} \mathbf{1}_{\lambda}\right)$ for all $\boldsymbol{i}, \boldsymbol{j}$ and $\lambda$. Then

$$
\operatorname{gdim} \operatorname{HOM}_{u}\left(\varepsilon_{\boldsymbol{i}} \mathbf{1}_{\lambda}, \mathcal{E}_{\boldsymbol{j}} \mathbf{1}_{\lambda}\right)=\sum_{t \in \mathbb{Z}} q^{t} \operatorname{dim}_{\mathbb{k}} \dot{u}\left(\mathcal{E}_{\boldsymbol{i}} \mathbf{1}_{\lambda}\{t\}, \mathcal{E}_{\boldsymbol{j}} \mathbf{1}_{\lambda}\right) .
$$

Since the calculus is non-degenerate,

$$
\operatorname{gdim} \operatorname{HOM}_{u}\left(\varepsilon_{\boldsymbol{i}} \mathbf{1}_{\lambda}, \boldsymbol{E}_{\boldsymbol{j}} \mathbf{1}_{\lambda}\right)=\pi\left\langle E_{\boldsymbol{i}} 1_{\lambda}, E_{\boldsymbol{j}} 1_{\lambda}\right\rangle,
$$

and $E_{\boldsymbol{i}} 1_{\lambda}$, over all $\boldsymbol{i}, \lambda$, span $\dot{\mathbf{U}}$, the $\mathbb{Q}(q)$-algebra homomorphism

$$
\gamma_{\mathbb{Q}(q)}: \dot{\mathbf{U}} \rightarrow K_{0}(\dot{U}) \otimes_{\mathbb{Z}\left[q, q^{-1}\right]} \mathbb{Q}(q)
$$

intertwines the $\mathbb{Q}(q)$-semilinear forms $\pi\langle$,$\rangle on \dot{\mathbf{U}}$ and $\operatorname{gdim} \operatorname{HOM} u($,$) on K_{0}$. The latter form, which we denote $\langle,\rangle_{\pi}$, is given by

$$
\langle[P],[Q]\rangle_{\pi}:=\sum_{t \in \mathbb{Z}} q^{t} \operatorname{gdim}(\dot{u}(P\{t\}, Q))
$$


for any two 1-morphisms $P, Q \in \dot{U}(\lambda, \mu)$, and extends to the entire $K_{0}(\dot{U}) \otimes_{\mathbb{Z}\left[q, q^{-1}\right]}$ $\mathbb{Q}(q)$ via $\mathbb{Q}(q)$-semilinearity and the orthogonality condition $\langle x, y\rangle_{\pi}=0$ for $x \in$ $K_{0}(\dot{U}(\lambda, \mu)), y \in K_{0}\left(\dot{U}\left(\lambda^{\prime}, \mu^{\prime}\right)\right)$ unless $\lambda=\lambda^{\prime}$ and $\mu=\mu^{\prime}$.

By Proposition 2.5, $\langle$,$\rangle is non-degenerate on \dot{\mathbf{U}}$. Therefore, $\gamma_{\mathbb{Q}(q)}$ is injective, implying that $\gamma$ is injective.

\section{Categorification of $\dot{U}$ for $s \mathfrak{r}_{n}$}

4.1. Forms of quantum $\mathfrak{s I}_{\boldsymbol{n}}$. We consider various forms of the quantized enveloping algebra of $\mathfrak{s l}_{n}$ corresponding to the root datum of the Dynkin graph

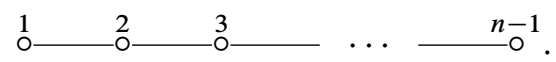

For this root datum, any weight $\lambda \in X$ can be written as $\lambda=\left(\lambda_{1}, \lambda_{2}, \ldots, \lambda_{n-1}\right)$, where $\lambda_{i}=\langle i, \lambda\rangle$.

The algebra $\left.\mathbf{U}_{q}(\mathfrak{s})_{n}\right)$ is the $\mathbb{Q}(q)$-algebra with 1 generated by the elements $E_{i}$, $F_{i}$ and $K_{i}^{ \pm 1}$ for $i=1,2, \ldots, n-1$, with the defining relations

$$
\begin{aligned}
K_{i} K_{i}^{-1} & =K_{i}^{-1} K_{i}=1, & K_{i} K_{j} & =K_{j} K_{i}, \\
K_{i} E_{j} K_{i}^{-1} & =q^{i \cdot j} E_{j}, & K_{i} F_{j} K_{i}^{-1} & =q^{-i \cdot j} F_{j},
\end{aligned}
$$

where $i \cdot i=2, i \cdot j=-1$ if $j=i \pm 1$ and $i \cdot j=0$ otherwise,

$$
\begin{gathered}
E_{i} F_{j}-F_{j} E_{i}=\delta_{i j} \frac{K_{i}-K_{i}^{-1}}{q-q^{-1}}, \\
E_{i}^{2} E_{j}-\left(q+q^{-1}\right) E_{i} E_{j} E_{i}+E_{j} E_{i}^{2}=0 \text { if } j=i \pm 1, \\
F_{i}^{2} F_{j}-\left(q+q^{-1}\right) F_{i} F_{j} F_{i}+F_{j} F_{i}^{2}=0 \text { if } j=i \pm 1, \\
E_{i} E_{j}=E_{j} E_{i}, F_{i} F_{j}=F_{j} F_{i} \text { if }|i-j|>1 .
\end{gathered}
$$

Recall that $\dot{\mathbf{U}}\left(\mathfrak{s}_{n}\right)$ is obtained from $\mathbf{U}_{q}\left(\mathfrak{s l}_{n}\right)$ by adjoining a collection of orthogonal idempotents $1_{\lambda}$ indexed by the weight lattice $X$ of $\mathfrak{s}{ }_{n}$,

$$
1_{\lambda} 1_{\lambda^{\prime}}=\delta_{\lambda \lambda^{\prime}} 1_{\lambda}
$$

such that if $\lambda=\left(\lambda_{1}, \lambda_{2}, \ldots, \lambda_{n-1}\right)$, then

$$
K_{i} 1_{\lambda}=1_{\lambda} K_{i}=q^{\lambda_{i}} 1_{\lambda}, \quad E_{i} 1_{\lambda}=1_{\lambda+i_{X}} E_{i}, \quad F_{i} 1_{\lambda}=1_{\lambda-i_{X}} F_{i},
$$

where

$$
\lambda+i_{X}= \begin{cases}\left(\lambda_{1}+2, \lambda_{2}-1, \lambda_{3}, \ldots, \lambda_{n-2}, \lambda_{n-1}\right) & \text { if } i=1, \\ \left(\lambda_{1}, \lambda_{2}, \ldots, \lambda_{n-2}, \lambda_{n-1}-1, \lambda_{n-1}+2\right) & \text { if } i=n-1, \\ \left(\lambda_{1}, \ldots, \lambda_{i-1}-1, \lambda_{i}+2, \lambda_{i+1}-1, \ldots, \lambda_{n-1}\right) & \text { otherwise }\end{cases}
$$




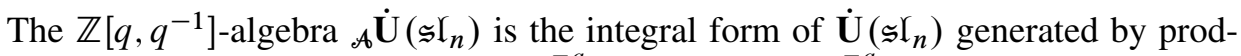
ucts of divided powers $E_{i}^{(a)} 1_{\lambda}:=\frac{E_{i}^{a}}{[a] !} 1_{\lambda}, F_{i}^{(a)} 1_{\lambda}:=\frac{F_{i}^{a}}{[a] !} 1_{\lambda}$ for $\lambda \in X$ and $i=$ $1,2, \ldots, n-1$. The relationships are collected below:

$$
\mathbf{U}_{q}\left(\mathfrak{s} \mathfrak{l}_{n}\right) \stackrel{\begin{array}{c}
\text { add } \\
\text { idempotents }
\end{array}}{\longrightarrow} \dot{\mathbf{U}}\left(\mathfrak{s} \mathfrak{l}_{n}\right) \stackrel{\begin{array}{c}
\text { integral } \\
\text { form }
\end{array}}{\longleftarrow} \dot{\mathbf{U}}\left(\mathfrak{s} \mathfrak{l}_{n}\right)
$$

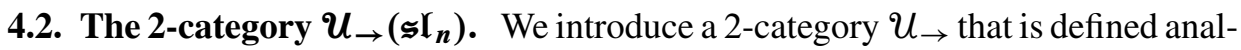
ogously to $\mathcal{U}$ in the $s \mathfrak{I}_{n}$-case, but the $R(v)$-relations have been modified to the signed $R(v)$-relations given in [17]. Namely, the $R(v)$-relations in $\mathcal{U}$ are replaced in $\mathcal{U}_{\rightarrow}$ by the signed $R(v)$-relations obtained from the oriented graph

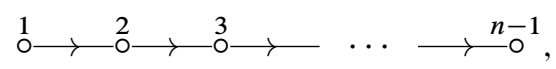

with vertices enumerated by the set $\{1,2, \ldots, n-1\}$, using signs $\tau_{i j}=\tau_{j i}=-1$ for all edges. It was observed in [17] that the resulting ring $R_{\tau}(v)$ is isomorphic to $R(v)$. In Section 4.2.1, following the definition of $U_{\rightarrow}$, we extend this isomorphism to an isomorphism $U \rightarrow U_{\rightarrow}$ of 2 -categories. The 2 -category $U_{\rightarrow}$ is more convenient for constructing a representation on iterated flag varieties in Section 6.

In general it is a poor practice to set up an isomorphism rather than an equivalence of categories, not to mention 2-categories. However, having an isomorphism $u \rightarrow$ $U_{\rightarrow}$ is justified, since $U_{\text {and }} U_{\rightarrow}$ have the same objects, morphisms, and generating 2-morphisms.

Definition 4.1. $U_{\rightarrow}\left(\mathfrak{s} \mathfrak{I}_{n}\right)$ is a additive $\mathbb{k}$-linear 2-category with translation. The 2category $\mathcal{U}_{\rightarrow}\left(\mathfrak{s} \mathfrak{l}_{n}\right)$ has objects, morphisms, and generating 2-morphisms as defined in (3.1), but some of the relations on 2-morphisms are modified.

- The $s \mathfrak{I}_{2}$ relations and the shift isomorphism relations are the same as before; see equations (3.1)-(3.9).

- All 2-morphisms are cyclic with respect to the biadjoint structure as before; see (3.3) and (3.10).

- The relations (3.13) hold.

- The signed $R(v)$ relations are:

(a) For $i \neq j$, the relations

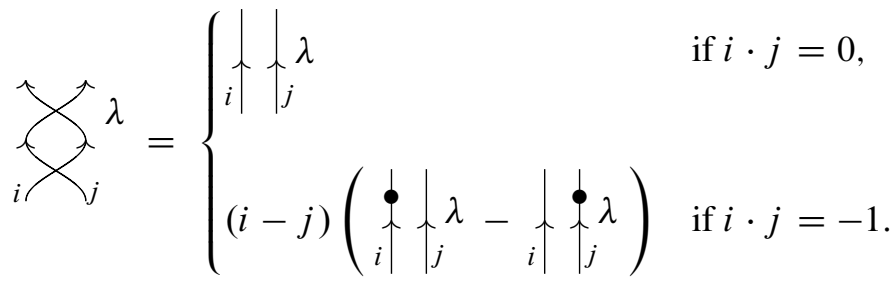


(b) For $i \neq j$, the relations

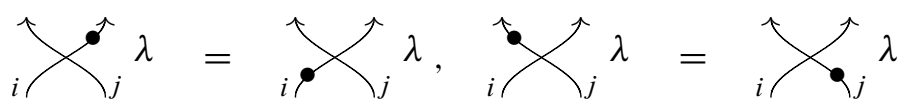

for all $\lambda$.

(c) Unless $i=k$ and $j=i \pm 1$

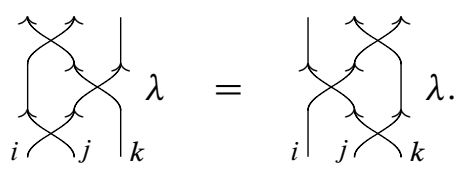

For $j=i \pm 1$

$$
\uparrow_{i \mid} \uparrow_{j} \lambda=(i-j)\left(\left.\sum_{i}^{\lambda} \lambda\right|_{i} \lambda-\sum_{j}^{\lambda} \lambda\right) .
$$

4.2.1. The 2-isomorphism $\Sigma: \mathcal{U} \rightarrow \boldsymbol{U}_{\rightarrow}$. Define an isomorphism of 2-categories $\Sigma: \mathcal{U} \rightarrow \mathcal{U}_{\rightarrow}$ on objects by mapping $\lambda \mapsto \lambda$, and on hom categories by graded additive $\mathbb{k}$-linear functors

$$
\begin{aligned}
& \Sigma: \mathcal{U}(\lambda, \mu) \longrightarrow U_{\rightarrow}(\lambda, \mu), \\
& \varepsilon_{i} \mathbf{1}_{\lambda} \longmapsto \varepsilon_{i} \mathbf{1}_{\lambda},
\end{aligned}
$$

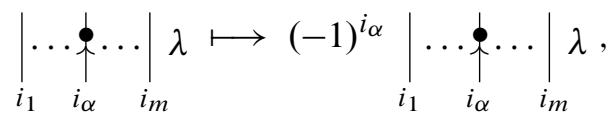

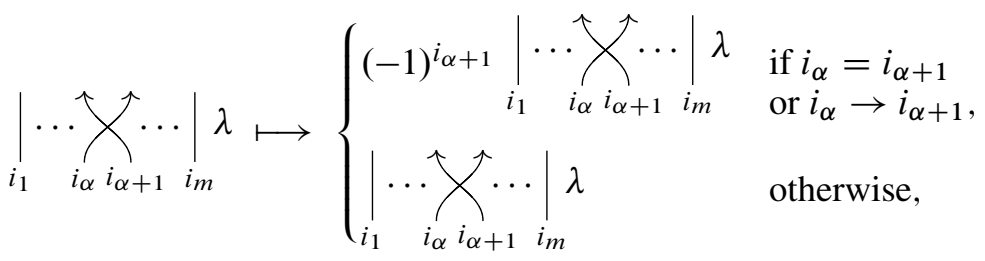

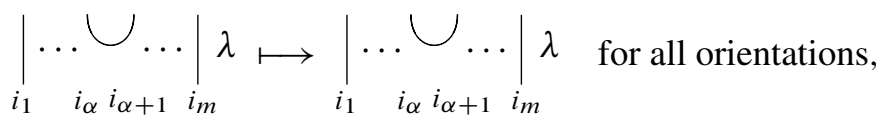

$$
\begin{aligned}
& \left.\left.\left.\right|_{i_{1}} \cdots \bigcap_{i_{\alpha} i_{\alpha+1}}^{\cdots} i_{m}^{\lambda \longmapsto}\right|_{i_{1}} \cdots \bigcap_{i_{\alpha}} \cdots{ }_{i_{\alpha+1}}^{\cdots}\right|_{i_{m}} ^{\lambda} \text { for all orientations. }
\end{aligned}
$$


Above, the $i_{\alpha}$ in $(-1)^{i_{\alpha}}$ refers to the enumeration of the vertex $i_{\alpha}$ in (4.2). One can check that the above transformations respects the $\mathfrak{s t}_{2}$-relations and the cyclic condition. Furthermore, $\Sigma$ maps the $R(v)$-relations to their signed analogs by rescaling the generators as above. $\Sigma$ is a 2 -functor and an isomorphism of 2-categories.

Remark 4.2. Since the 2-categories $U$ and $U_{\rightarrow}$ are isomorphic, by the universal property of the Karoubi envelope, their Karoubi envelopes are isomorphic as well.

Define $U_{\rightarrow}^{*}$ to be the graded additive $\mathbb{k}$-linear category which has the same objects and 1-morphisms as $U_{\rightarrow}$ and 2-morphisms

$$
u_{\rightarrow}^{*}(x, y):=\bigoplus_{t \in \mathbb{Z}} u_{\rightarrow}(x\{t\}, y) .
$$

4.2.2. Relation to rings $\boldsymbol{R}(v)$. Regard the graded $\mathbb{k}$-algebra $R(v)$ with system of idempotents $\left\{1_{\boldsymbol{i}}\right\}$ as a pre-additive $\mathbb{k}$-linear category whose objects are $\{\boldsymbol{i} \mid \boldsymbol{i} \in$ $\operatorname{Seq}(v)\}$. The $\mathbb{k}$-vector space of morphisms from $\boldsymbol{i}$ to $\boldsymbol{i}^{\prime}$ is ${ }_{\boldsymbol{i}}^{\prime} R(v)_{\boldsymbol{i}}$. The composition $i^{\prime \prime} R(v)_{\boldsymbol{i}^{\prime}} \otimes \boldsymbol{i}^{\prime} R(v)_{\boldsymbol{i}} \rightarrow \boldsymbol{i}^{\prime \prime} R(v)_{\boldsymbol{i}}$ is given by multiplication in $R(v)$.

For any weight $\lambda$ there is a graded additive $\mathbb{k}$-linear functor

$$
\iota_{\lambda}: R(v) \longrightarrow U_{\rightarrow}^{*}\left(\lambda, \lambda+v_{X}\right)
$$

that takes object $\boldsymbol{i}$ to $\mathcal{E}_{\boldsymbol{i}} \mathbf{1}_{\lambda}$, and is given on generators of homs by

$$
\begin{aligned}
& { }_{j} R(v)_{i} \longrightarrow u_{\rightarrow}^{*}\left(\varepsilon_{i} \mathbf{1}_{\lambda}, \mathcal{E}_{j} \mathbf{1}_{\lambda}\right),
\end{aligned}
$$

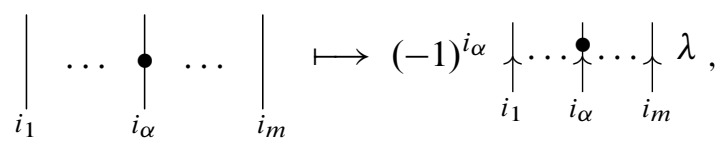

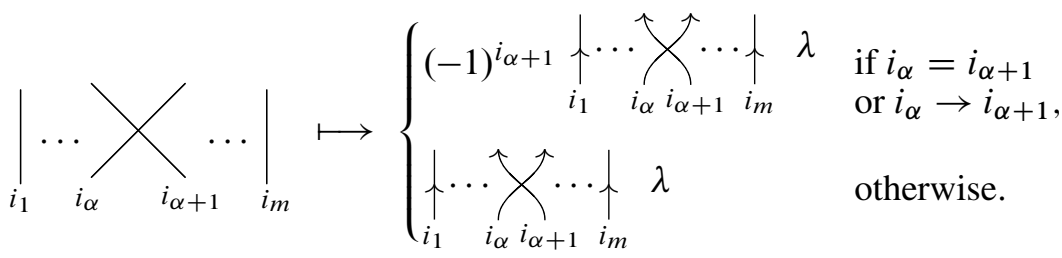

Linear combination of diagrams in $R(v)$ get sent to the corresponding rescaled linear combination in $U_{\rightarrow}^{*}\left(\lambda, \lambda+v_{X}\right)$ with the weight $\lambda$ labelling the far right region. In what follows we will refer to those diagrams in the image of $\iota_{\lambda}$ as $R(v)$ generators. The image of $R(v)$ is spanned by diagrams with all strands upward pointing and no caps or cups. 


\section{Iterated flag varieties}

5.1. Cohomology of $\boldsymbol{n}$-step varieties. The material in this section generalizes that of [21, Section 6]. The reader is encouraged to start there for more examples and greater detail in the $\mathfrak{s} \Upsilon_{2}$ case. We enumerate by $I=\{1,2, \ldots, n-1\}$ the vertex set of the Dynkin diagram of $\mathfrak{s}_{n}$,

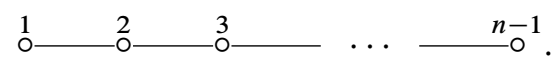

Fix $N \geq 0$, and consider the variety $\mathrm{Fl}(n)$ of $n$-step partial flags $F$

$$
F=\left(0=F_{0} \subseteq F_{1} \subseteq \cdots \subseteq F_{n}=\mathbb{C}^{N}\right)
$$

in $\mathbb{C}^{N}$. The dimensions of the subspaces $F_{i}$ are conveniently expressed as a vector,

$$
\underline{\operatorname{dim} F}=\left(\operatorname{dim} F_{0}, \operatorname{dim} F_{1}, \operatorname{dim} F_{2}, \ldots, \operatorname{dim} F_{n}\right) .
$$

The connected components of $\mathrm{Fl}(n)$ are parameterized by non-negative integers

$$
\underline{k}=\left(k_{0}, k_{1}, k_{2}, \ldots, k_{n}\right)
$$

such that $0=k_{0} \leq k_{1} \leq k_{2} \leq \cdots \leq k_{n}=N$. The connected component $\operatorname{Fl}(\underline{k})$

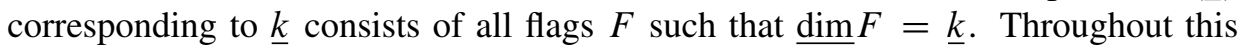
section we refer to the terms $k_{\alpha}$ of $\underline{k}$ with the convention that

$$
k_{\alpha}= \begin{cases}0 & \text { if } \alpha \leq 0 \\ N & \text { if } \alpha \geq n .\end{cases}
$$

The cohomology algebra of $\mathrm{Fl}(\underline{k})$ is $\mathbb{Z}_{+}$-graded,

$$
H_{\underline{k}}:=H^{*}(\mathrm{Fl}(\underline{k}), \mathbb{k})=\bigoplus_{0 \leq \ell \leq k_{1}\left(k_{2}-k_{1}\right) \ldots\left(N-k_{n-1}\right)} H^{2 \ell}(\mathrm{Fl}(\underline{k}), \mathbb{k}) .
$$

For $1 \leq j \leq n, 0<\alpha \leq k_{j}-k_{j-1}$, let $x(\underline{k})_{j, \alpha}$ be a formal variable of degree $2 \alpha$. The ring $H_{\underline{k}}$ is isomorphic to the quotient ring

$$
\left(\bigotimes_{j=1}^{n} \mathbb{k}\left[x(\underline{k})_{j, 1}, x(\underline{k})_{j, 2}, \ldots, x(\underline{k})_{j, k_{j}-k_{j-1}}\right]\right) / I_{\underline{k}, N},
$$

where $I_{\underline{k}, N}$ is the ideal generated by the homogeneous terms in the equation

$$
\prod_{j=1}^{n}\left(1+x(\underline{k})_{j, 1} t+x(\underline{k})_{j, 2} t^{2}+\cdots+x(\underline{k})_{j, k_{j}-k_{j-1}} t^{k_{j}-k_{j-1}}\right)=1 .
$$

Above, $t$ is a formal variable used to keep track of the degrees. For notational convenience we add variables $x(\underline{k})_{j, 0}$ and set $x(\underline{k})_{j, 0}=1$. Furthermore, we set

$$
x(\underline{k})_{j, \alpha}=0 \quad \text { if } \alpha>k_{j}-k_{j-1} .
$$


It is helpful to express the above relation in an alternative form. Let $\overline{x(\underline{k})_{i, \alpha}}$ denote the homogeneous term of degree $2 \alpha$ in the product

$$
\prod_{j=1, j \neq i}^{n}\left(1+x(\underline{k})_{j, 1} t+x(\underline{k})_{j, 2} t^{2}+\cdots+x(\underline{k})_{j, k_{j}-k_{j-1}} t^{k_{j}-k_{j-1}}\right) .
$$

For example, if $n=4$ and $\underline{k}=(1,3,4,7)$, then equation (5.1) becomes

$$
\begin{aligned}
&\left(1+x(\underline{k})_{1,1} t\right)\left(1+x(\underline{k})_{2,1} t\right.\left.+x(\underline{k})_{2,2} t^{2}\right)\left(1+x(\underline{k})_{3,1} t\right) \\
&\left(1+x(\underline{k})_{4,1} t+x(\underline{k})_{4,2} t^{2}+x(\underline{k})_{4,3} t^{3}\right)=1
\end{aligned}
$$

and the terms $\overline{x(\underline{k})_{2, \alpha}}$ are given by omitting the second term and multiplying out the rest

$$
\left(1+x(\underline{k})_{1,1} t\right)\left(1+x(\underline{k})_{3,1} t\right)\left(1+x(\underline{k})_{4,1} t+x(\underline{k})_{4,2} t^{2}+x(\underline{k})_{4,3} t^{3}\right)
$$

so that

$$
\begin{aligned}
\overline{x(\underline{k})_{2,0}}= & 1, \\
\overline{x(\underline{k})_{2,1}}= & x(\underline{k})_{1,1}+x(\underline{k})_{3,1}+x(\underline{k})_{4,1}, \\
\overline{x(\underline{k})_{2,2}}= & x(\underline{k})_{1,1} x(\underline{k})_{3,1}+x(\underline{k})_{1,1} x(\underline{k})_{4,1}+x(\underline{k})_{3,1} x(\underline{k})_{4,1}+x(\underline{k})_{4,2}, \\
\overline{x(\underline{k})_{2,3}=} & x(\underline{k})_{1,1} x(\underline{k})_{3,1} x(\underline{k})_{4,1}+x(\underline{k})_{1,1} x(\underline{k})_{4,2} \\
& +x(\underline{k})_{3,1} x(\underline{k})_{4,2}+x(\underline{k})_{4,3}, \\
\overline{x(\underline{k})_{2,4}}= & x(\underline{k})_{1,1} x(\underline{k})_{3,1} x(\underline{k})_{4,2}+x(\underline{k})_{1,1} x(\underline{k})_{4,3}+x(\underline{k})_{3,1} x(\underline{k})_{4,3}, \\
\overline{x(\underline{k})_{2,5}}= & x(\underline{k})_{1,1} x(\underline{k})_{3,1} x(\underline{k})_{4,3},
\end{aligned}
$$

and $\overline{x(\underline{k})_{2, \alpha}}=0$ for $\alpha>5$. It is clear that (5.1) can be written

$$
\sum_{f=0}^{\alpha} x(\underline{k})_{j, f} \overline{x(\underline{k})_{j, \alpha-f}}=\delta_{\alpha, 0}
$$

for any $1 \leq j \leq n$, where $\delta_{\alpha, 0}$ is the Kronecker delta. We call the elements $\overline{x(\underline{k})_{j, \alpha}}$ dual generators in light of (5.4).

For $1 \leq i \leq n-1$ let

$$
\begin{aligned}
+i \underline{k} & =\left(\left(+{ }_{i} k\right)_{0},\left(+{ }_{i} k\right)_{1},\left(+{ }_{i} k\right)_{2}, \ldots,\left(+{ }_{i} k\right)_{n}\right), \\
0 & =\left(+{ }_{i} k\right)_{0} \leq(+i k)_{1} \leq \cdots \leq(+i k)_{n}=N,
\end{aligned}
$$

be the sequence obtained from the sequence $\underline{k}$ by increasing the $i$ th term by one

$$
+i \underline{k}:=\left(k_{0}, k_{1}, k_{2}, \ldots, k_{i-1}, k_{i}+1, k_{i+1}, \ldots, k_{n}\right)
$$


if $k_{i}+1 \leq k_{i+1}$, or by setting the sequence to the empty sequence $\varnothing$ if $k_{i}=k_{i+1}$. Namely, $\left({ }_{+i} k\right)_{j}=k_{j}$ if $j \neq i$ and $\left({ }_{+i} k\right)_{i}=k_{i}+1$ if $k_{i}+1 \leq k_{i+1}$.

When $k_{i}+1 \leq k_{i+1}$ then $H_{+i \underline{k}}$ is the cohomology ring of the partial flag variety consisting of flags $F$ with $\underline{\operatorname{dim}} F=+i \underline{k}$. The ring $H_{+i \underline{k}}$ is given by

$$
\begin{aligned}
H_{+i} \underline{k}= & \left.\left.\bigotimes_{j=1}^{n} \mathbb{k}\left[x(+i \underline{k})_{j, 1}, x(+i \underline{k})_{j, 2}, \ldots, x(+i \underline{k})_{j,(+i} k\right)_{j}-(+i k)_{j-1}\right]\right) / I_{+i} \underline{k}, N \\
= & \bigotimes_{j \neq i, i+1} \mathbb{k}\left[x(+i \underline{k})_{j, 1}, \ldots, x(+i \underline{k})_{j, k_{j}-k_{j-1}}\right] \\
& \otimes \mathbb{k}\left[x(+i \underline{k})_{i, 1}, \ldots, x(+i \underline{k})_{k_{i}-k_{i-1}+1}\right] \\
& \otimes \mathbb{k}\left[x(+i \underline{k})_{i+1,1}, \ldots, x(+i \underline{k})_{k_{i+1}-k_{i}-1}\right] / I_{+i} \underline{k}, N,
\end{aligned}
$$

where $I_{+i} \underline{k}, N$ is the ideal generated by the homogeneous terms in

$$
\prod_{j=1}^{n}\left(\sum_{\alpha \geq 0} x\left({ }_{+i} \underline{k}\right)_{j, \alpha} t^{\alpha}\right)=1 .
$$

We define $H_{\emptyset}=0$.

Going back to the example of $n=4$ and $\underline{k}=(1,3,4,7)$, then $+3 \underline{k}=(1,3,5,7)$, so that

$$
\begin{aligned}
H_{+3} \underline{k}=\mathbb{k} & {\left[x(+3 \underline{k})_{1,1}, x(+3 \underline{k})_{2,1}, x(+3 \underline{k})_{2,2},\right.} \\
& \left.\cdot x(+3 \underline{k})_{3,1}, x(+3 \underline{k})_{3,2}, x(+3 \underline{k})_{4,1}, x(+3 \underline{k})_{4,2}\right] / I_{+3} \underline{k}, 7,
\end{aligned}
$$

where $I_{+3 \underline{k}, 7}$ is the ideal generated by the homogeneous terms in

$$
\begin{aligned}
\left(1+x(+3 \underline{k})_{1,1}\right)(1 & \left.+x(+3 \underline{k})_{2,1}+x(+3 \underline{k})_{2,2}\right)\left(1+x(+3 \underline{k})_{3,1}\right. \\
& \left.+x(+3 \underline{k})_{3,2}\right)\left(1+x(+3 \underline{k})_{4,1}+x(+3 \underline{k})_{4,2}\right)=1 .
\end{aligned}
$$

Similarly, we write $-i \underline{k}=\left(k_{0}, k_{1}, k_{2}, \ldots, k_{i-1}, k_{i}-1, k_{i+1}, \ldots, k_{n}\right)$ for the sequence $\underline{k}$ where we have subtracted one from the $i$ th position whenever $k_{i-1} \leq$ $k_{i}-1$. The cohomology ring of the flag variety $\mathrm{Fl}(-i \underline{k})$ is $H_{-i} \underline{k}$, which can be expressed explicitly in terms of generators as above. When $k_{i-1}=\bar{k}_{i}$ then ${ }_{-i} \underline{k}=\emptyset$ and $H_{-i \underline{k}}:=H_{\emptyset}=0$.

5.1.1. Flag varieties for the action of $\boldsymbol{E}_{\boldsymbol{i}}$ and $\boldsymbol{F}_{\boldsymbol{i}}$. For $1 \leq i \leq n-1$, define

$$
\begin{aligned}
& \underline{k}^{+i}= \begin{cases}\left(k_{0}, k_{1}, k_{2}, \ldots k_{i}, k_{i}+1, k_{i+1}, \ldots, k_{n-1}, k_{n}\right) & \text { if } k_{i+1} \geq k_{i}+1, \\
\emptyset & \text { otherwise },\end{cases} \\
& \underline{k}^{-i}= \begin{cases}\left(k_{0}, k_{1}, k_{2}, \ldots k_{i-1}, k_{i}-1, k_{i}, \ldots, k_{n-1}, k_{n}\right) & \text { if } k_{i}-1 \geq k_{i-1}, \\
\emptyset & \text { otherwise } .\end{cases}
\end{aligned}
$$


For $\underline{k}^{ \pm i} \neq \varnothing$ the variety $\mathrm{Fl}\left(\underline{k}^{ \pm i}\right)$ is the component of $\mathrm{Fl}(n+1)$ consisting of flags $F$ such that $\underline{\operatorname{dim}} F=\underline{k}^{ \pm i}$. The cohomology ring of $\mathrm{Fl}\left(\underline{k}^{ \pm i}\right)$ will be denoted by $H_{\underline{k}}^{ \pm i}$. The cohomology ring $H_{\underline{k}}+i$ is

$$
\begin{aligned}
H_{\underline{k}}+i & =\bigotimes_{j \neq i+1} \mathbb{k}\left[x\left(\underline{k}^{+i}\right)_{j, 1}, \ldots, x\left(\underline{k}^{+i}\right)_{\left.j, k_{j}-k_{j-1}\right]}\right] \otimes \mathbb{k}\left[\xi_{i}\right] \\
& \otimes \mathbb{k}\left[x\left(\underline{k}^{+i}\right)_{i+1,1}, \ldots, x\left(\underline{k}^{+i}\right)_{i+1, k_{i+1}-k_{i}-1}\right] / I_{\underline{k}^{+i}, N},
\end{aligned}
$$

where $I_{\underline{k}^{+i}, N}$ is the ideal generated by the homogeneous terms in

$$
\begin{aligned}
\left(1+\xi_{i} t\right)\left(1+x\left(\underline{k}^{+i}\right)_{i+1,1} t+\ldots+\right. & \left.x\left(\underline{k}^{+i}\right)_{i+1, k_{i+1}-k_{i}-1} t^{k_{i+1}-k_{i}-1}\right) \\
& \cdot \prod_{j \neq i+1}\left(\sum_{f=0}^{k_{j}-k_{j-1}} x\left(\underline{k}^{+i}\right)_{j, f} t^{f}\right)=1 .
\end{aligned}
$$

The forgetful maps

$$
\mathrm{Fl}(\underline{k}) \stackrel{p_{1}}{\longleftarrow} \mathrm{Fl}\left(\underline{k}^{+i}\right) \stackrel{p_{2}}{\longrightarrow} \mathrm{Fl}(+i \underline{k})
$$

induce maps of cohomology rings

$$
H_{\underline{k}} \stackrel{p_{1}^{*}}{\longrightarrow} H_{\underline{k}}+i \stackrel{p_{2}^{*}}{\longleftarrow} H_{+i \underline{k}}
$$

that make $H_{k}+i$ a right $H_{\underline{k}} \otimes H_{+i} \underline{k}$-module. Since the algebra $H_{+i \underline{k}}$ is commutative, we can turn a right $H_{+i} \underline{k}$-module into a left $H_{+i \underline{k}}$-module. Hence, we can make $H_{\underline{k}}{ }^{+i}$ into a $\left(H_{+i \underline{k}}, H_{\underline{k}}\right)$-bimodule. In fact, $H_{k^{+i}}$ is free as a graded $H_{\underline{k}}$-module and as a graded $H_{+i \underline{k}}$-module.

These inclusions making $H_{\underline{k}}+i$ a $\left(H_{+i \underline{k}}, H_{\underline{k}}\right)$-bimodule are given explicitly as follows:

$$
\begin{aligned}
H_{\underline{k}} & \longmapsto H_{\underline{k}^{+i}}, \\
x(\underline{k})_{j, \alpha} & \longmapsto x\left(\underline{k}^{+i}\right)_{j, \alpha} \text { for } j \neq i+1, \\
x(\underline{k})_{i+1, \alpha} & \longmapsto \xi_{i} \cdot x\left(\underline{k}^{+i}\right)_{i+1, \alpha-1}+x\left(\underline{k}^{+i}\right)_{i+1, \alpha},
\end{aligned}
$$

and

$$
\begin{aligned}
H_{+i \underline{k}} & \longmapsto H_{\underline{k}^{+i}}, \\
x(+i \underline{k})_{j, \alpha} & \longmapsto x\left(\underline{k}^{+i}\right)_{j, \alpha} \text { for } j \neq i, \\
x(+i \underline{k})_{i, \alpha} & \longmapsto \xi_{i} \cdot x\left(\underline{k}^{+i}\right)_{i, \alpha-1}+x\left(\underline{k}^{+i}\right)_{i, \alpha} .
\end{aligned}
$$

Notice that $x(\underline{k})_{j, \alpha}$ and $x(+i \underline{k})_{j, \alpha}$ for $j \neq i, i+1$ are mapped to the same element of $H_{\underline{k}}+i$. Using these inclusions we identify these elements of $H_{\underline{k}}$ and $H_{+i \underline{k}}$ with 
their images in the bimodule $H_{\underline{k}}+i$. Furthermore, we can also express the generators $x\left(\underline{k}^{+i}\right)_{i, \alpha}$ and $x\left(\underline{k}^{+i}\right)_{i+1, \beta}$ of $H_{\underline{k}^{+i}}$ as the images of certain generators in $H_{\underline{k}}$ or $H_{+i \underline{k}}$. Thus we can write $H_{\underline{k}}+i$ as

$$
H_{\underline{k}^{+i}}=\bigotimes_{j \neq i+1} \mathbb{k}\left[x(\underline{k})_{j, 1}, \ldots, x(\underline{k})_{j, k_{j}-k_{j-1}}\right]
$$

$\otimes \mathbb{k}\left[x(+i \underline{k})_{i+1,1}, \ldots, x(+i \underline{k})_{i+1, k_{i+1}-k_{i}-1}\right] \otimes \mathbb{k}\left[\xi_{i}\right] / I_{\underline{k}^{+i}, N}$

or equivalently

$$
\begin{aligned}
H_{\underline{k}}+i & =\bigotimes_{j \neq i} \mathbb{k}\left[x(+i \underline{k})_{j, 1}, \ldots, x(+i \underline{k})_{j, k_{j}-k_{j-1}}\right] \\
& \otimes \mathbb{k}\left[x(\underline{k})_{i, 1}, \ldots, x(\underline{k})_{i, k_{i}-k_{i-1}}\right] \otimes \mathbb{k}\left[\xi_{i}\right] / I_{\underline{k}^{+i}, N},
\end{aligned}
$$

where $I_{\underline{k}^{+i}, N}$ is the ideal described above. Therefore, $\xi_{i}$ is the only generator of $H_{\underline{k}}{ }^{+i}$ that is not identified with a generator of $H_{\underline{k}}$ or $H_{+i \underline{k}}$ under the above inclusions.

Remark 5.1. The generators $x(\underline{k})_{j, \alpha}, x(+i \underline{k})_{j, \alpha}, \xi_{i}$ of $H_{\underline{k}}+i$ correspond to Chern classes of tautological bundles over the variety $\mathrm{Fl}\left(\underline{k}^{+i}\right)$. The generator $\xi_{i}$ corresponds to the line bundle $F_{k_{i}+1} / F_{k_{i}}$ associated to the subspaces $F_{k_{i}} \subset F_{k_{i}+1}$ created by the subspace insertion $\underline{k}^{+i}$.

Definition 5.2. The set of multiplicative generators $\xi_{i}$ and

$$
\begin{aligned}
x(\underline{k})_{i, \alpha_{i}} & \text { for } 0<\alpha_{i} \leq k_{i}-k_{i-1}, \\
x(+i \underline{k})_{i+1, \alpha_{i+1}} & \text { for } 0<\alpha_{i+1} \leq k_{i+1}-k_{i}-1, \\
x(\underline{k})_{j, \alpha_{j}}=x(+i \underline{k})_{j, \alpha_{j}} \in H_{\underline{k}}+i & \text { for } j \neq i, i+1, \text { and } 0<\alpha_{j} \leq k_{j}-k_{j-1},
\end{aligned}
$$

for the ring $H_{k^{+i}}$, corresponding to the Chern class of the tautological line bundle, and to the canonical inclusions (5.5) and (5.6) of generators in $H_{+i \underline{k}}$ and $H_{\underline{k}}$ into $H_{\underline{k}^{+i}}$, are called the canonical generators of $H_{\underline{k}}+i$.

Using commutativity we can regard $H_{\underline{k}}+i$ as an $\left(H_{\underline{k}}, H_{+i \underline{k}}\right)$-bimodule. The generators of $H_{\underline{k}}$ and $H_{+i \underline{k}}$ that are not mapped to canonical generators can be expressed in terms of canonical generators as follows:

$$
\begin{aligned}
x(\underline{k})_{i+1, \alpha} & =\xi_{i} \cdot x(+i \underline{k})_{i+1, \alpha-1}+x(+i \underline{k})_{i+1, \alpha}, \\
x(+i \underline{k})_{i, \alpha} & =\xi_{i} \cdot x(\underline{k})_{i, \alpha-1}+x(\underline{k})_{i, \alpha},
\end{aligned}
$$

for all values of $\alpha$. 


\subsection{Graphical calculus for iterated flag varieties}

5.2.1. Rings $\boldsymbol{H}_{\underline{k}}$. All of what has been described can be easily visualized using a shorthand notation in which the generators of $H_{k}$ are drawn as labelled bubbles floating in a region carrying a label $\lambda$ called the weight. The label $\lambda$ will be important when we relate partial flag varieties to the 2-category $\dot{U}$ categorifying ${ }_{\mathcal{A}} \dot{\mathbf{U}}\left(\mathfrak{s} \mathfrak{I}_{n}\right)$. More precisely, $\lambda$ will correspond to a weight of the irreducible representation of ${ }_{\mathcal{A}} \dot{\mathbf{U}}\left(\mathfrak{s} \mathrm{I}_{n}\right)$ with highest weight $(N, 0,0, \ldots, 0)$.

To a sequence $\underline{k}=\left(k_{0}, k_{1}, k_{2}, \ldots, k_{n}\right)$ as above associate

$$
\lambda=\lambda(\underline{k})=\left(\lambda_{1}, \lambda_{2}, \ldots, \lambda_{n-1}\right) \in \mathbb{Z}^{n-1}
$$

where

$$
\lambda_{\alpha}=-k_{\alpha+1}+2 k_{\alpha}-k_{\alpha-1} .
$$

The weight corresponding to the sequence $+i \underline{k}$ is defined analogously, where $k_{i}$ is replaced by $k_{i}+1$. Comparing with (4.1) it is clear that $\lambda(+i \underline{k})=\lambda+i_{X}$. Similarly, $\lambda(-i \underline{k})=\lambda-i_{X}$.

With this convention, the generators of $H_{\underline{k}}$ and $H_{+i \underline{k}}$ are depicted as

$$
\begin{aligned}
x(\underline{k})_{j, \alpha} & :=\underbrace{\lambda}_{j, \alpha} \\
x(+i \underline{k})_{j, \alpha} & :=i^{\lambda, \alpha}
\end{aligned}
$$

where the identity is depicted by the empty region of the appropriate weight. Products of generators are depicted by a bubble in the plane for each generator present in the product. Diagrams are only considered up to planar isotopy. A generic element in $H_{\underline{k}}$ can be depicted as a formal linear combination of such diagrams. For example, if $n=4, \underline{k}=(1,3,4,7)$ then the element $x(\underline{k})_{1,1} x(\underline{k})_{4,3}+5 \cdot x(\underline{k})_{3,1} \in H_{\underline{k}}$ is represented as

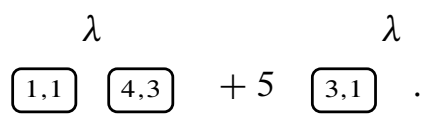

If we depict the dual generators $\overline{x(\underline{k})_{j, \alpha}}$ of $H_{\underline{k}}$ defined in (5.3) as

$$
\overline{x(\underline{k})_{j, \alpha}}=j^{\lambda, \alpha}
$$

then the defining relations (5.4) for $H_{\underline{k}}$ become the equations

$$
\sum_{f=0}^{\alpha} j_{j, f}^{\lambda}=\sum_{g=0}^{\alpha} j_{j, \alpha-f}^{\lambda}=\delta_{\alpha, 0}^{\lambda}
$$


for $\alpha \geq 0$ and $1 \leq j \leq n$. Notice that

$$
{ }_{j, 0}^{\lambda}=b^{\lambda}=1, \quad{ }^{\lambda, \alpha}=0
$$

if $\alpha<0$, or $k_{j}-k_{j-1}<\alpha$; see (5.2) and the comments preceding (5.2).

5.2.2. Bimodules $\boldsymbol{H}_{\boldsymbol{k}^{+i}}$. The identity element in $H_{k^{+i}}$ is represented by a vertical line labelled $i$,

$$
H_{\underline{k}+i} \ni 1:=\lambda+\left.i_{X}\right|_{i} \lambda,
$$

where the orientation indicates that we are regarding $H_{\underline{k}}+i$ as an $\left(H_{+i \underline{k}}, H_{\underline{k}}\right)$-bimodule. The $\lambda$ on the right-hand side is the weight corresponding to $\underline{k}$. Hence, having $\lambda$ on the right-hand side of the diagram indicates the right action of $H_{\underline{k}}$ on $H_{\underline{k}}+i$. Similarly, the $\lambda+i_{X}$ on the left indicates the left action of $H_{+i \underline{k}}$ on $H_{k}+i$.

When regarding $H_{k^{+i}}$ as an $\left(H_{\underline{k}}, H_{+i} \underline{k}\right)$-bimodule we depict it in the graphical calculus with the opposite orientation (a downward pointing arrow).

$$
H_{\underline{k}+i} \ni 1:=\left.\lambda\right|_{i} \lambda+i_{X} .
$$

We will often omit the weights from all regions but one, with it understood that crossing an upward pointing arrow with label $i$ from right to left changes the weight by $i_{X}$, and crossing a downward pointing arrow from right to left changes the weight by $-i_{X}$.

Equations (5.7) show that all of the generators from $H_{k}+i$ except for $\xi_{i}$ can be interpreted as either generators of $H_{\underline{k}}$ or $H_{+i} \underline{k}$ under the natural inclusions. This fact is represented in the graphical calculus as follows:

$$
\begin{aligned}
H_{\underline{k}+i} \ni x(\underline{k})_{j, \alpha} & :=\uparrow_{i}^{j, \alpha}{ }^{\lambda}, \quad j \neq i+1, \\
H_{\underline{k}+i} \ni x(+i \underline{k})_{j, \beta} & :=\left.\right|_{i} ^{\lambda,}, \quad j \neq i, \\
H_{\underline{k}+i} \ni \xi_{i} & :=\oint_{i}^{\lambda},
\end{aligned}
$$

where each of the diagram inherits a grading from the Chern class it represents $\left(\operatorname{deg} x(\underline{k})_{j, \alpha}=2 \alpha, \operatorname{deg} x(+i \underline{k})_{j, \beta}=2 \beta\right.$, and $\left.\operatorname{deg} \xi_{i}=2\right)$. Equation (5.10) is meant to depicts the generator $x(\underline{k})_{j, \alpha} \in H_{\underline{k}}+i$ as the element $x(\underline{k})_{j, \alpha} \in H_{\underline{k}}$ acting on 
the identity of $H_{k^{+i}}$. Likewise, the generator $x(+i \underline{k})_{j, \beta} \in H_{k^{+i}}$ is depicted as the element $x(+i \underline{\underline{k}})_{j, \beta} \in H_{+i} \underline{k}$ acting on the identity of $H_{k}+i$. The generator $\xi_{i}$ is represented by a dotted line so that $\xi_{i}^{\alpha}$ is represented by $\alpha$ dots on a line, but for simplicity we write this using a single dot and a label to indicate the power.

The identification (5.5) and (5.6) of $x(\underline{k})_{j, \alpha}$ with $x(+i \underline{k})_{j, \alpha}$ in $H_{k^{+i}}$ for $j \neq i, i+1$ leads to the graphical identity

$$
\uparrow_{i}^{j, \alpha}{ }^{\lambda}=\uparrow_{i}^{\lambda} \quad \text { for } j \neq i, i+1 .
$$

Similarly, (5.8) provides the identities

$$
\begin{aligned}
& \int_{i}^{i+1, \alpha}{ }^{\lambda}=\left.\underbrace{i+1, \alpha-1}_{i}\right|^{\lambda}+\left.\underbrace{i+1, \alpha}_{i}\right|_{i} ^{\lambda} \\
& \left.\left.\right|_{i} ^{\lambda, \alpha}\right|_{i, \alpha-1} ^{\lambda}+\uparrow_{i}{ }_{i, \alpha}^{\lambda}
\end{aligned}
$$

expressing non-canonical generators in terms of canonical generators. It is sometimes helpful to express the canonical generators in terms of non-canonical generators:

$$
\begin{aligned}
& \left.\underbrace{i+1, \alpha}_{i}\right|_{f=0} ^{\lambda}=\left.\sum_{i=1}^{\alpha}(-1)^{f}\right|_{i} ^{f} \underbrace{\lambda}_{i+1, \alpha-f} \\
& \uparrow_{i}^{i, \alpha}{ }^{\lambda}=\left.\sum_{f=0}^{\alpha}(-1)^{f} \underbrace{i, \alpha-f}_{i}\right|^{\lambda},
\end{aligned}
$$

which can be verified using (5.12) and (5.13). From these equations we can derive other useful identities:

$$
\begin{aligned}
& \oint_{i}^{\alpha} \lambda=(-1)^{\alpha} \sum_{f=0}^{\alpha} i_{i}^{i+1, \alpha-f} \uparrow_{i+1, f}^{\lambda} \\
& \oint_{i}^{\alpha \lambda}=(-1)^{\alpha} \sum_{g=0}^{\alpha} i, \alpha-g \uparrow_{i} i, g \text {. }
\end{aligned}
$$




\section{Proposition 5.3.}

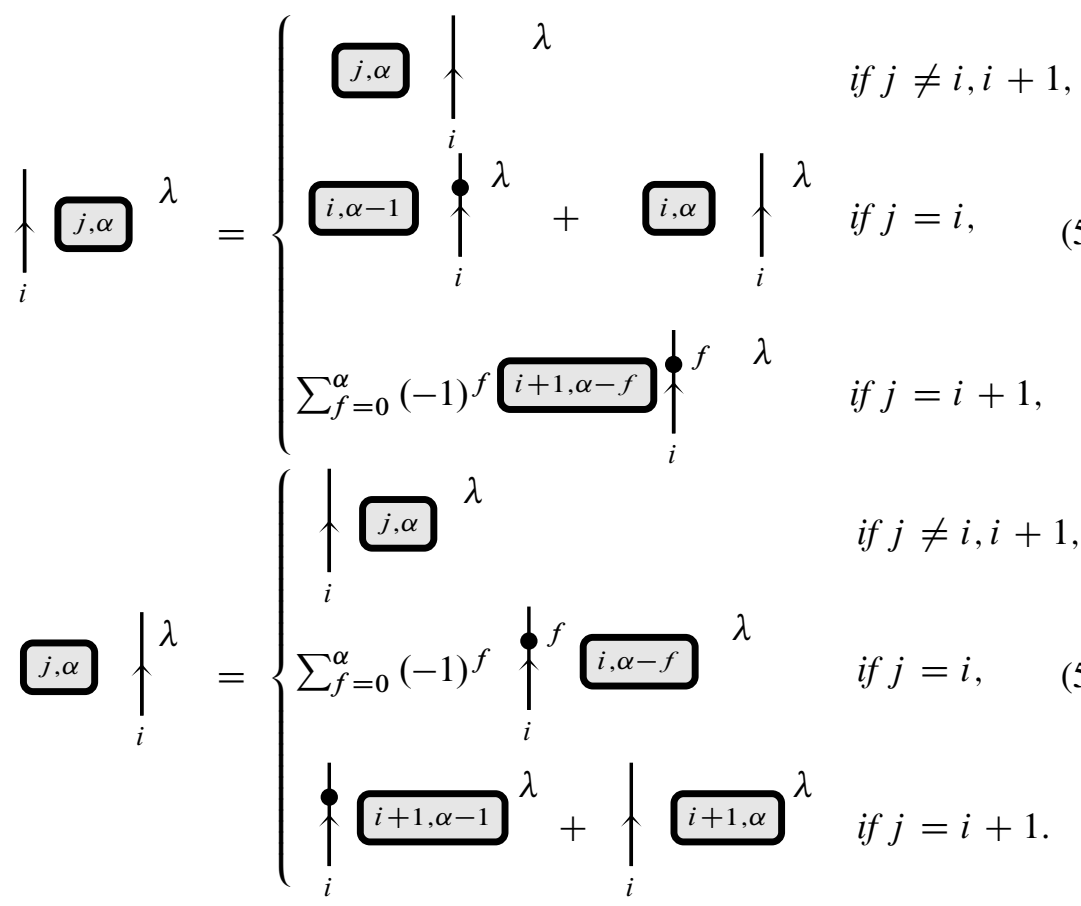

Proof. Recall that the elements $\overline{x(\underline{k})_{j, \alpha}}$ are sums of homogeneous symmetric terms in all variables except for $x(\underline{k})_{j, \beta}$. By (5.11) all terms $x(\underline{k})_{\ell, \alpha}$ for $\ell \neq i, i+1$ can be slid across the line labelled $i$. The case when $j \neq i, i+1$ then reduces to the problem of sliding symmetric combinations $\sum_{f=0}^{\beta} x(\underline{k})_{i, f} x(\underline{k})_{i+1, \beta-f}$ across a line labelled $i$. Such slides are determined by the following calculation in $H_{k^{+i}}$ :

$$
\begin{aligned}
& \sum_{f=0}^{\beta} x(\underline{k})_{i, f} x(\underline{k})_{i+1, \beta-f} \\
& \stackrel{(5.15)}{=} \sum_{f=0}^{\beta} \sum_{g=0}^{f}(-1)^{g} x(+i \underline{k})_{i, f-g} \xi_{i}^{g} x(\underline{k})_{i+1, \beta-f} \\
& \stackrel{(5.12)}{=} \sum_{f=0}^{\beta} \sum_{g=0}^{f}(-1)^{g} x(+i \underline{k})_{i, f-g} x(+i \underline{k})_{i+1, \beta-f-1} \xi_{i}^{g+1} \\
& +\sum_{f=0}^{\beta} \sum_{g=0}^{f}(-1)^{g} x(+i \underline{k})_{i, f-g} x(+i \underline{k})_{i+1, \beta-f} \xi_{i}^{g} .
\end{aligned}
$$

Change variables to $f^{\prime}=f+1$, and $g^{\prime}=g+1$ in the first summation, so that all 
terms cancel except for $g=0$ term in the second summation; this term is equal to

$$
\sum_{f=0}^{\beta} x(+i \underline{k})_{i, f} x(+i \underline{k})_{i+1, \beta-f} .
$$

Hence, when $j \neq i, i+1$ the elements $\overline{x(\underline{k})_{j, \alpha}}$ and $\overline{x(+i \underline{k})_{j, \alpha}}$ can be slid across a line labelled $i$.

The dual generator $\overline{x(\underline{k})_{j, \alpha}}$ contains symmetric homogeneous combinations of variables $x(\underline{k})_{\ell, \beta}$ for $\ell \neq j$. When $j=i$ in (5.18) all terms in $\overline{x(\underline{k})_{i, \alpha}}$ slide across lines labelled $i$ except for the variables $x(\underline{k})_{i+1, \beta}$. Using (5.12) to slide these across establishes (5.18) for the case $j=i$. Similarly, when $j=i+1$ all terms in $\overline{x(\underline{k})_{i+1, \alpha}}$ slide from right to left across lines labelled $i$ except for the variables $x(\underline{k})_{i, \beta}$. Using (5.15) to slide these completes the proof of (5.18). Equation (5.19) is proven similarly.

By duality, analogous equations as those above hold for downward pointing arrows. For example, equation (5.17) implies

$$
\lambda \oint_{i}^{\alpha}=(-1)^{\alpha} \sum_{g=0}^{\alpha} \lambda i, i, g \uparrow_{i} i, \alpha-g .
$$

5.2.3. Bimodules $\boldsymbol{H}_{\boldsymbol{k}^{\boldsymbol{i}}}$. For the remainder of this paper we write a signed sequence $\boldsymbol{i}=\varepsilon_{1} i_{1} \varepsilon_{2} i_{2} \ldots \varepsilon_{m} i_{m}$ as $\boldsymbol{i}=s_{1} s_{2} \ldots s_{m}$ with $s_{\alpha}=\varepsilon_{\alpha} i_{\alpha}$. For $s_{\alpha}=\varepsilon_{\alpha} i_{\alpha}$ write $s_{\alpha} \underline{k}$ for the sequence obtained from $\underline{k}=\left(k_{0}, k_{1}, k_{2}, \ldots, k_{n}\right)$ by increasing the $k_{i_{\alpha}}$ by 1 if $\varepsilon_{\alpha}=+$ and $k_{i_{\alpha}}<k_{i_{\alpha+1}}$, decreasing the sequence by 1 if $\varepsilon_{\alpha}=-$ and $k_{i_{\alpha-1}}<k_{i_{\alpha}}$, and setting the sequence to $\emptyset$ otherwise. Then $i \underline{k}$ is either set to $\emptyset$, or else it denotes the sequence obtained from $\underline{k}$ with the $\left(i_{\alpha}\right)$ th term increased by one if $s_{\alpha}=+i_{\alpha}$, or decreased by one if $s_{\alpha}=-i_{\alpha}$, sequentially for each $s_{\alpha}$ in the signed sequence $\boldsymbol{i}$, reading from the right. It is clear that the sequence $i \underline{k}$ is equal to the sequence $j \underline{k}$ whenever $\boldsymbol{i}, \boldsymbol{j} \in$ SSeq with $\boldsymbol{i}_{X}=\boldsymbol{j}_{X} \in X$ and $\boldsymbol{i} \underline{k} \neq \varnothing$ and $\boldsymbol{j} \underline{k} \neq \varnothing$.

We write $H_{\underline{k}} i$ for the $\left(H_{i \underline{k}}, H_{\underline{k}}\right)$-bimodule

$$
H_{\underline{k}^{i}}:=H_{s_{2} s_{3} \ldots s_{m}} \underline{k}^{s_{1}} \otimes_{H_{s_{2} s_{3} \ldots s_{m} \underline{k}}} \cdots \otimes_{H_{s_{m-1} s_{m} \underline{k}}} H_{s_{m}} \underline{\underline{k}}^{s_{m-1}} \otimes_{H_{s_{m} \underline{k}}} H_{\underline{k}^{s m}} .
$$

This bimodule can also be described as the cohomology ring of the variety of $m+n$ step iterated partial flags corresponding to the sequence obtained from $\underline{k}$ by the ordered insertion of subspaces determined from the signed sequence $\boldsymbol{i}$.

The $\left(H_{i \underline{k}}, H_{\underline{k}}\right)$-bimodule $H_{\underline{k}}$ can be understood using the graphical calculus. A general signed sequence $\boldsymbol{i}=s_{1} s_{2} \ldots s_{m}$ is represented by a sequence of lines coloured by the sequence $i_{1} i_{2} \ldots i_{m}$, where the line coloured by $i_{\alpha}$ is oriented upward if $s_{\alpha}=+i_{\alpha}$ and oriented downward if $s_{\alpha}=-i_{\alpha}$. 
Examples. (1) For the signed sequence $+j+i \in \mathrm{SSeq}$ consider the $\left(H_{+j+i}, H_{\underline{k}}\right)$ bimodule

$$
H_{\underline{k}^{+j+i}}=H_{+i \underline{k}} \underline{+j}^{+j} \otimes_{H_{+i} \underline{k}} H_{\underline{k}^{+i}} .
$$

As explained in the previous section, the identity elements of the $\left(H_{+i} \underline{k}, H_{\underline{k}}\right)$-bimodule $H_{\underline{k}^{+i}}$ and the $\left(H_{+i+j \underline{k}}, H_{+i \underline{k}}\right)$-bimodule $H_{+i \underline{\underline{k}}}{ }^{j}$ are depicted as

$$
\lambda+i_{X} \uparrow_{i} \lambda \quad \text { and } \lambda+i_{X}+\left.j_{X}\right|_{j} \lambda+i_{X}
$$

respectively.

The identity element of $H_{\underline{k}^{+j+i}}$ is represented by the diagram

$$
\lambda+i_{X}+\left.j_{X}\right|_{j} \lambda+\left.i_{X}\right|_{i} \lambda .
$$

The region in the middle of the two lines is labelled by the weight $\lambda+i_{X}$ corresponding to $+i \underline{k}$. The tensor product over the action of $H_{+i \underline{k}}$ is represented diagrammatically by the fact that a labelled bubble in the region with weight $\lambda+i_{X}$ can be equivalently regarded as an element of $H_{+i \underline{k}}$ acting on the line corresponding to $H_{\underline{k}^{+i}}$, or the line corresponding to $H_{+i} \underline{k}^{j}$.

$$
\left.\uparrow_{j} \uparrow_{i}\right|_{\ell, \alpha} ^{\lambda} \quad \uparrow_{j}^{\ell, \alpha} \uparrow_{i}^{\lambda} \quad \text { Action of } H_{+i \underline{k}} \quad \text { Action of } H_{+j+i \underline{k}} \uparrow_{i}^{\lambda} .
$$

The weight $\lambda$ on the far right, and the weight of $\lambda+i_{X}+j_{X}$ on the far left, indicate that this diagram is describing an $\left(H_{+j+i \underline{k}}, H_{\underline{k}}\right)$-bimodule where the various actions are depicted as above.

(2) For $\boldsymbol{i}=+i_{1}+i_{2}+i_{3} \cdots+i_{r}$ the identity element of the bimodule $H_{\underline{k}^{i}}$ is depicted by a sequence of upward oriented labelled lines

$$
H_{\underline{k}^{i}} \ni 1=\uparrow_{i_{1}} \uparrow_{i_{2}} \uparrow_{i_{3}} \cdots \uparrow_{i_{m-1}} \uparrow_{i_{m}}^{\lambda} .
$$

The canonical generators $\xi_{i_{\alpha}}$ of each term in the tensor product (5.21) are represented graphically by a dot on the line labelled $i_{\alpha}$,

$$
H_{\underline{k}^{i}} \ni 1 \otimes 1 \otimes \ldots 1 \otimes \xi_{i_{\alpha}} \otimes 1 \cdots \otimes 1=\uparrow_{i_{1}} \uparrow_{i_{2}} \cdots \uparrow_{i_{\alpha}} \uparrow_{i_{m}} \lambda .
$$


Likewise, the tensor product over the rings $H_{s_{\alpha} s_{\alpha+1} \ldots s_{m} \underline{k}}$ is represented by the regions between lines. Again, the weights on the far left and right of the diagram indicate the bimodule structure.

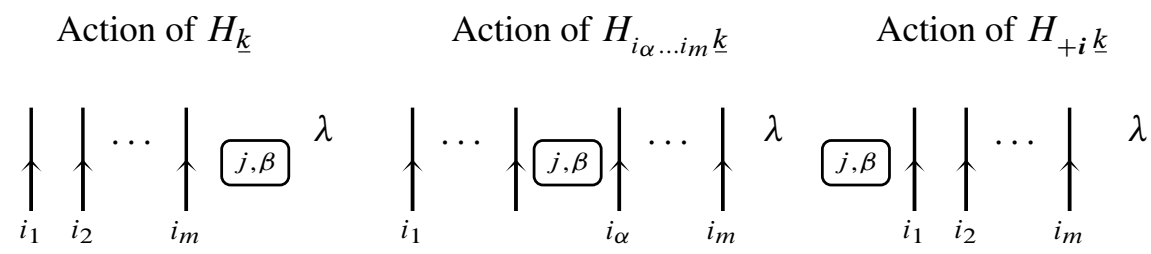

(3) Consider the $\left(H_{\underline{k}}, H_{\underline{k}}\right)$-bimodule corresponding to the tensor product

$$
H_{\underline{k}}^{+i} \otimes_{H_{+i} \underline{k}} H_{\underline{k}}+i
$$

where in the first factor we are regarding $H_{k}+i$ as a $\left(H_{\underline{k}}, H_{+i} \underline{k}\right)$-bimodule. The identity element of this bimodule is represented by the diagram

$$
\left.\lambda\right|_{i} \lambda+\left.i_{X}\right|_{i} \lambda .
$$

5.2.4. Identities arising from tensor products. Bubbles with a given label $(j, \alpha)$ can pass from right to left, or left to right, through a line coloured by $i$ as long as $j \neq i, i+1$. If $j=i$ or $j=i+1$ then a bubble can move through a line subject to the rules (5.12)-(5.15). Furthermore, dots on a line can be exchanged for bubbles in the neighboring regions using (5.16) and (5.17). Dual bubbles corresponding to dual generators can be slid across lines using the rules (5.18) and (5.19).

The following Lemma is needed for the definition of the 2-representation $\Gamma$ given in the next section. In particular, parts (i) and (ii) are used to provide two equivalent definitions of

$$
\Gamma\left(\succ_{i} \lambda_{\lambda}\right), \Gamma\left(\succ_{i} \gamma_{\lambda}\right),
$$

and (iii) and (iv) are used to show that

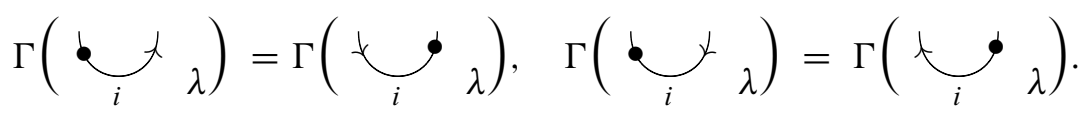

Lemma 5.4. The following identities hold.

$$
\begin{aligned}
& \sum_{f=0}^{\alpha}(-1)^{\alpha-f} \oint_{i}^{f} \uparrow{ }_{i}^{i, \alpha-f}{ }^{\lambda}=\left.\sum_{g=0}^{\alpha}(-1)^{\alpha-g} \sqrt{i, \alpha-g}\right|_{i} \oint_{i}^{g} \lambda, \\
& \sum_{f=0}^{\alpha}(-1)^{\alpha-f} \xi_{i}^{f} \otimes x(\underline{k})_{i, \alpha-f}=\sum_{g=0}^{\alpha}(-1)^{\alpha-g} x(\underline{k})_{i, \alpha-g} \otimes \xi_{i}^{g}
\end{aligned}
$$


for all $\alpha \in \mathbb{N}$.

ii) In the ring $H_{-i \underline{k}}^{+i} \otimes_{H_{-i} \underline{k}} H_{-i \underline{k}} \underline{+i}^{i}$ we have

$$
\begin{aligned}
\left.\left.\sum_{f=0}^{\alpha}(-1)^{\alpha-f}\right|_{i} ^{f}\right|_{i} ^{i+1, \alpha-f}{ }^{\lambda} & =\left.\sum_{g=0}^{\alpha}(-1)^{\alpha-g} \underbrace{i+1, \alpha-g}_{i}\right|_{i} \oint^{g \lambda}, \\
\sum_{f=0}^{\alpha}(-1)^{\alpha-f} \xi_{i}^{f} \otimes x(\underline{k})_{i+1, \alpha-f} & =\sum_{g=0}^{\alpha}(-1)^{\alpha-g} x(\underline{k})_{i+1, \alpha-g} \otimes \xi_{i}^{g}
\end{aligned}
$$

for all $\alpha \in \mathbb{N}$.

iii) Dot slide formulas for cups: in the ring $H_{\underline{k}}^{+i} \otimes_{H_{+i} \underline{\underline{k}}} H_{\underline{\underline{k}}+i}$ we have

$$
\begin{aligned}
\sum_{f=0}^{k_{i}-k_{i-1}}(-1)^{k_{i}-k_{i-1}-f} \xi_{i}^{f+1} \otimes x(\underline{k})_{i+1, k_{i}-k_{i-1}-f} \\
\quad=\sum_{g=0}^{k_{i}-k_{i-1}}(-1)^{k_{i}-k_{i-1}-g} \xi_{i}^{g} \otimes x(\underline{k})_{i+1, k_{i}-k_{i-1}-g} \cdot \xi_{i} .
\end{aligned}
$$

iv) Dot slide formulas for cups: In the ring $H_{-i \underline{k}} \underline{x}^{+i} \otimes_{H_{-i} \underline{\underline{k}}} H_{-i \underline{k}}+i$ we have

$$
\begin{aligned}
\sum_{f=0}^{k_{i+1}-k_{i}}(-1)^{k_{i+1}-k_{i}-f} \xi_{i}^{f+1} \otimes x(\underline{k})_{i+1, k_{i+1}-k_{i}-f} \\
\quad=\sum_{g=0}^{k_{i+1}-k_{i}}(-1)^{k_{i+1}-k_{i}-g} \xi_{i}^{g} \otimes x(\underline{k})_{i+1, k_{i+1}-k_{i}-g} \cdot \xi_{i} .
\end{aligned}
$$

Proof. Part i) follows from the chain of equalities below

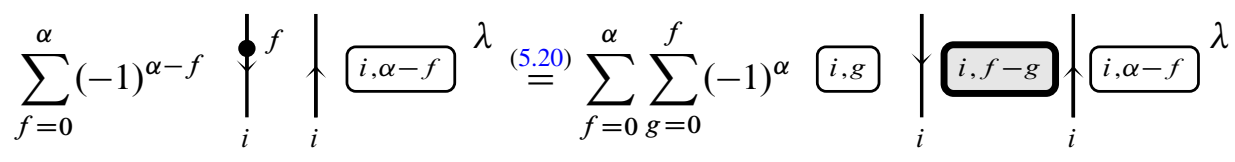

If we re-index by letting $f^{\prime}=\alpha-f$ and switch the order of summation we have

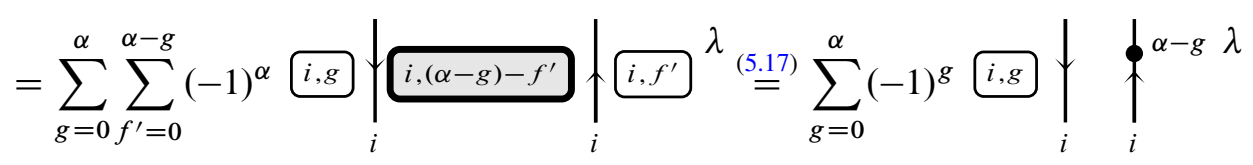

which after re-indexing completes the proof of part i). Part ii) is proven similarly. Part iii) follows from part i) by letting $f^{\prime}=f+1$ and adding and subtracting the 
term $(-1)^{\alpha+1} 1 \otimes x(\underline{k})_{i, \alpha+1}$, so that

$$
\begin{aligned}
\sum_{f=0}^{\alpha}(-1)^{\alpha-f} \xi_{i}^{f+1} \otimes x(\underline{k})_{i, \alpha-f} & \\
\quad= & \sum_{f^{\prime}=0}^{\alpha+1}(-1)^{\alpha+1-f^{\prime}} \xi_{i}^{f^{\prime}} \otimes x(\underline{k})_{i, \alpha+1-f^{\prime}}-(-1)^{\alpha+1} 1 \otimes x(\underline{k})_{i, \alpha+1} \\
& \stackrel{\text { part i })}{=} \sum_{g=0}^{\alpha+1}(-1)^{\alpha+1-g} x(\underline{k})_{i, \alpha+1-g} \otimes \xi_{i}^{g}-(-1)^{\alpha+1} 1 \otimes x(\underline{k})_{i, \alpha+1} .
\end{aligned}
$$

Pulling off the $g=0$ term and re-indexing $g^{\prime}=g-1$ we have

$$
\sum_{g^{\prime}=0}^{\alpha}(-1)^{\alpha-g^{\prime}} x(\underline{k})_{i, \alpha-g^{\prime}} \otimes \xi_{i}^{g^{\prime}+1}+(-1)^{\alpha+1}\left(x(\underline{k})_{i, \alpha+1} \otimes 1-1 \otimes x(\underline{k})_{i, \alpha+1}\right) .
$$

But the term with the summation is equal to

$$
\sum_{g^{\prime}=0}^{\alpha}(-1)^{\alpha-g^{\prime}} \xi_{i}^{g^{\prime}} \otimes x(\underline{k})_{i, \alpha-g^{\prime}} \cdot \xi_{i}
$$

by part i), and the remaining terms $(-1)^{\alpha+1}\left(x(\underline{k})_{i, \alpha+1} \otimes 1-1 \otimes x(\underline{k})_{i, \alpha+1}\right)$, are zero when $\alpha=k_{i}-k_{i-1}$ since $x(\underline{k})_{i, \alpha+1}$ is zero for $\alpha \geq k_{i}-k_{i-1}$, see (5.2). Part iv) is proven similarly using part ii).

Corollary 5.5. The assignments (see Definition 6.1)

$$
\begin{aligned}
\Gamma\left(\bigcup_{i, \lambda}\right): H_{\underline{k}} & \longrightarrow\left(H_{\underline{k}^{+i}} \otimes_{H_{+i} \underline{\underline{k}}} H_{\underline{k}^{+i}}\right)\left\{1+k_{i-1}-k_{i+1}\right\}, \\
1 & \longmapsto \sum_{f=0}^{k_{i}-k_{i-1}}(-1)^{k_{i}-k_{i-1}-f} \xi_{i}^{f} \otimes x(\underline{k})_{i, k_{i}-k_{i-1}-f},
\end{aligned}
$$

and

$$
\begin{aligned}
\Gamma\left(\bigcup_{i, \lambda}\right): H_{\underline{k}} & \longrightarrow\left(H_{-i \underline{k^{+}}} \otimes_{H_{-i \underline{k}}} H_{-i \underline{k}}+i\right)\left\{1+k_{i-1}-k_{i+1}\right\}, \\
1 & \longmapsto \sum_{g=0}^{k_{i}+1-k_{i}}(-1)^{k_{i+1}-k_{i}-g} \xi_{i}^{g} \otimes x(\underline{k})_{i+1, k_{i+1}-k_{i}-g},
\end{aligned}
$$

define morphisms of graded bimodules of degree $1+\lambda_{i}=1-k_{i-1}+2 k_{i}-k_{i+1}$ and $1-\lambda_{i}=1+k_{i-1}-2 k_{i}+k_{i+1}$, respectively.

Proof. For the first claim it suffices to check that the left action of each generator of $H_{\underline{\underline{k}}}$ on $\Gamma\left(\bigcup_{i, \lambda}\right)(1) \in\left(H_{\underline{\underline{k}}}^{+i} \otimes_{H_{+i \underline{\underline{k}}}} H_{\underline{\underline{k}}}+i\right)$ is equal to right action of this generator. The Corollary follows since bubbles can slide across lines by (5.12)-(5.15) at the cost of introducing powers of $\xi_{i}$ on one of the tensor factors; by Lemma 5.4 (iii) and (iv), factors of $\xi_{i}$ can be slid across tensor factors in the above sums. The second claim is proven similarly and the degrees of these bimodule maps are easily computed. 
5.3. The 2-category Flag $_{N}$. Bim is a 2-category whose objects are graded rings, whose 1-morphisms are graded bimodules, and 2-morphisms are degree-preserving bimodule homomorphisms. Idempotent bimodule homomorphisms split in Bim, so that any 2-representation $\Psi: U \rightarrow \mathbf{B i m}$ extends uniquely (up to isomorphisms) to a 2-representation $\dot{U} \rightarrow$ Bim; see Section 3.4.

Graded 2-homs between graded bimodules $M_{1}$ and $M_{2}$ are given by

$$
\operatorname{HOM}_{\text {Bim }}\left(M_{1}, M_{2}\right):=\bigoplus_{t \in \mathbb{Z}} \operatorname{Hom}_{\mathbf{B i m}}\left(M_{1}\{t\}, M_{2}\right)
$$

Let Bim* be the 2-category with the same objects and 1-morphisms as Bim and 2-morphisms given by

$$
\operatorname{Bim}^{*}\left(M_{1}, M_{2}\right):=\operatorname{HOM}_{\mathbf{B i m}}\left(M_{1}, M_{2}\right) .
$$

We now define a sub 2-category Flag $_{N}$ of the 2-category Bim for each integer $N \in \mathbb{Z}_{+}$.

Definition 5.6. The additive $\mathbb{k}$-linear 2-category Flag $_{N}$ is the idempotent completion inside of Bim of the 2-category consisting of:

- Objects: the graded rings $H_{\underline{k}}$ for all $\underline{k}=\left(k_{0}, k_{1}, k_{2}, \ldots, k_{n}\right)$ with $0 \leq k_{1} \leq$ $k_{2} \cdots \leq k_{n}=N$.

- Morphisms: generated by the graded $\left(H_{k}, H_{k}\right)$-bimodule $H_{k}$, the graded $\left(H_{+i \underline{k}}, H_{\underline{k}}\right)$-bimodules $H_{\underline{k}}+i$ and the graded $\left(H_{\underline{k}}, H_{+i \underline{k}}\right)$-bimodule $H_{\underline{k}^{+i}}$ for all $i \in I$, together with their shifts $H_{k}\{t\}, H_{k}+i\{t\}$, and $H_{k}+i\{t\}$ for $t \in \mathbb{Z}$. The bimodules $H_{k}=H_{k}\{0\}$ are the identity 1-morphisms. Thus, a morphism from $H_{\underline{k}}$ to $H_{i \underline{k}}$ is a finite direct sum of graded $\left(H_{j}, H_{\underline{k}}\right)$-bimodules of the form

$$
H_{\underline{k}}^{j}\{t\}:=H_{s_{2} s_{3} \ldots s_{m} \underline{k}^{s_{1}}} \otimes_{H_{s_{2} s_{3} \ldots s_{m} \underline{k}}} \cdots \otimes_{H_{s_{m-1} s_{m} \underline{k}}} H_{s_{m} \underline{k}^{s_{m-1}}} \otimes_{H_{s_{m} \underline{k}}} H_{\underline{k}^{s m}}\{t\}
$$

for signed sequences $\boldsymbol{j}=s_{1} s_{2} \ldots s_{m}$ with $\boldsymbol{i}_{X}=\boldsymbol{j}_{X} \in X$.

- 2-morphisms: degree-preserving bimodule maps.

There is a graded additive subcategory Flag $_{N}^{*}$ of Bim* with the same objects and 1-morphisms as Flag $_{N}$, but with

$$
\operatorname{Flag}_{N}^{*}\left(M_{1}, M_{2}\right):=\bigoplus_{t \in \mathbb{Z}} \operatorname{Flag}_{N}\left(M_{1}\{t\}, M_{2}\right)
$$

In Section 6 we show that $\mathbf{F l a g}_{N}^{*}$ provides a 2-representation of $U_{\rightarrow}^{*}$; using the isomorphism $\Sigma: U^{*} \rightarrow U_{\rightarrow}^{*}$ and restricting to degree zero 2-morphisms, the subcategory Flag $_{N}$ provides a 2-representation of $U$. 


\section{Representing $u_{\rightarrow}^{*}$ on the flag 2-category}

In this section we define for each positive integer $N$ a 2-representation $\Gamma_{N}: U_{\rightarrow}^{*} \rightarrow$ Flag $_{N}^{*}$. The 2-functor $\Gamma_{N}$ is degree-preserving so that it restricts to a weak 2-functor $\Gamma: \mathcal{U} \rightarrow$ Flag $_{N}$. We will sometimes shorten $\Gamma_{N}$ to $\Gamma$ for simplicity.

6.1. Defining the 2-functor $\Gamma_{N}$. On objects the 2-representation $\Gamma_{N}: u_{\rightarrow}^{*} \rightarrow$ $\operatorname{Flag}_{N}^{*}$ sends $\lambda=\left(\lambda_{1}, \lambda_{2}, \ldots, \lambda_{n-1}\right)$ to the ring $H_{\underline{k}}$ when $\lambda=\lambda(\underline{k})$, i.e., when $\lambda_{\alpha}=-k_{\alpha+1}+2 k_{\alpha}-k_{\alpha-1}$.

$$
\begin{aligned}
\Gamma_{N}: U_{\rightarrow} & \rightarrow \text { Flag }_{N}^{*} \\
\lambda & \mapsto \begin{cases}H_{\underline{k}} & \text { if } \lambda=\lambda(\underline{k}), \\
0 & \text { otherwise. }\end{cases}
\end{aligned}
$$

Morphisms of $U_{\rightarrow}$ get mapped by $\Gamma_{N}$ to graded bimodules:

$$
\begin{aligned}
\Gamma_{N}: U_{\rightarrow} & \rightarrow \text { Flag }_{N}^{*}, \\
\mathbf{1}_{\lambda}\{t\} & \mapsto \begin{cases}H_{\underline{k}}\{t\} & \text { if } \lambda_{\alpha}=-k_{\alpha+1}+2 k_{\alpha}-k_{\alpha-1}, \\
0 & \text { otherwise, }\end{cases} \\
\mathcal{E}_{+i} \mathbf{1}_{\lambda}\{t\} & \mapsto \begin{cases}H_{\underline{k}^{+i}}\left\{t+1+k_{i-1}+k_{i}-k_{i+1}\right\} & \text { if } \lambda_{\alpha}=-k_{\alpha+1}+2 k_{\alpha}-k_{\alpha-1}, \\
0 & \text { otherwise, }\end{cases} \\
\mathcal{E}_{-i} \mathbf{1}_{\lambda}\{t\} & \mapsto \begin{cases}H_{\underline{k}^{-i}}\left\{t+1-k_{i}\right\} & \text { if } \lambda_{\alpha}=-k_{\alpha+1}+2 k_{\alpha}-k_{\alpha-1}, \\
0 & \text { otherwise. }\end{cases}
\end{aligned}
$$
so that

Here $H_{\underline{k}^{-i}}\left\{t+1-k_{i}\right\}$ is the bimodule $H_{\underline{k}^{-i}}$ with the grading shifted by $t+1-k_{i}$

$$
\left.\left(H_{\underline{k}}+i\left\{t+1-k_{i}\right\}\right)_{j}=\left(H_{\underline{k}}+i\right)_{j-\left(t+1-k_{i}\right.}\right) .
$$

More generally, the 1-morphism

$$
\mathcal{E}_{\boldsymbol{i}} \mathbf{1}_{\lambda}\{t\}=\mathcal{E}_{s_{1}} \mathbf{1}_{\lambda+\left(s_{2}\right)_{X}+\cdots+\left(s_{m-1}\right)_{X}+\left(s_{m}\right)_{X}} \circ \cdots \circ \mathcal{E}_{s_{m-1}} \mathbf{1}_{\lambda+\left(s_{m}\right)_{X}} \circ \mathcal{E}_{s_{m}} \mathbf{1}_{\lambda}\{t\}
$$

is mapped by $\Gamma_{N}$ to the graded bimodule $H_{\underline{k}}+i$ with grading shift $\left\{t+t^{\prime}\right\}$, where $t^{\prime}$ is the sum of the grading shifts for each terms of the composition in (6.1). Formal direct sums of morphisms of the above form are mapped to direct sums of the corresponding bimodules.

\subsubsection{Biadjointness}

Definition 6.1. The 2-morphisms generating biadjointness in $\mathcal{U}_{\rightarrow}^{*}$ are mapped by $\Gamma$ to the following bimodule maps. 
$\Gamma(\underbrace{f_{\lambda}}_{i}):\left\{\begin{aligned} H_{\underline{k}} & \longrightarrow\left(H_{\underline{k}}+i \otimes_{+i \underline{k}} H_{\underline{k}}+i\right)\left\{1+k_{i-1}-k_{i+1}\right\}, \\ \lambda & \left.\left.\longmapsto \sum_{f=0}^{k_{i}-k_{i-1}}(-1)^{k_{i}-k_{i-1}-f}\right|_{i} ^{f}\right|_{i, k_{i}-k_{i-1}-f} ^{\lambda}, \\ 1 \longmapsto & \sum_{f=0}^{k_{i}-k_{i-1}}(-1)^{k_{i}-k_{i-1}-f} \xi_{i}^{f} \otimes x(\underline{k})_{i, k_{i}-k_{i-1}-f},\end{aligned}\right.$

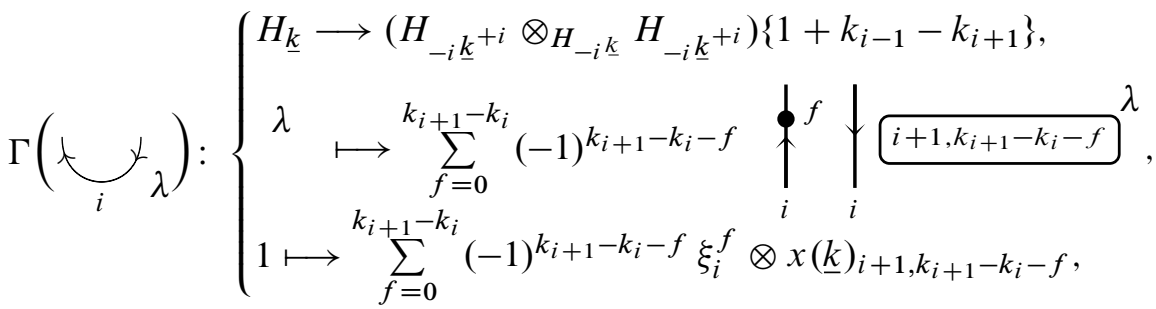

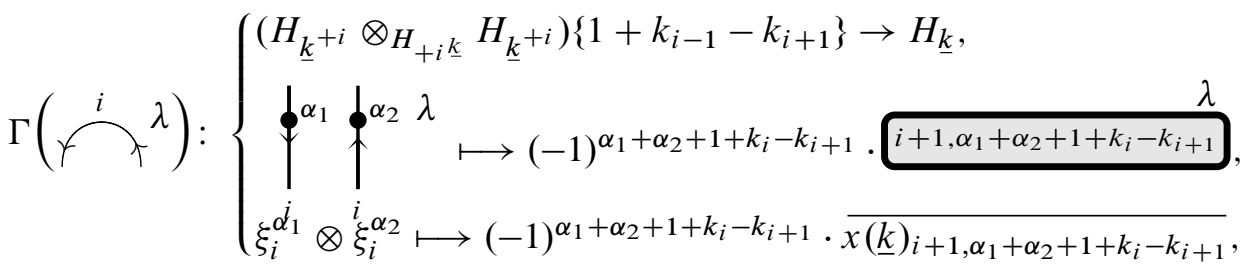

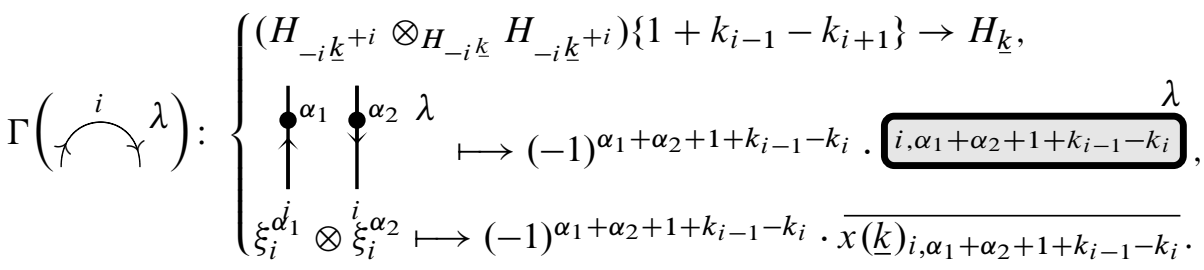

Corollary 5.5 shows that the cups above are bimodule maps. It is clear that the caps are bimodule maps. These definitions preserve the degree of the 2-morphisms of $u_{\rightarrow}^{*}$ defined in Section 3.1. By Lemma 5.4, the clockwise oriented cap and cup have degree $1-\lambda_{i}$ and the counter-clockwise oriented cap and cup have degree $1+\lambda_{i}$ so that these assignments are degree-preserving.

\subsection{2. $R(v)$ generators}

Definition 6.2. The 2-morphisms $\hat{\uparrow}_{i, \lambda}$ and $\downarrow_{i, \lambda}$ in $\mathcal{U}_{\rightarrow}^{*}$ are mapped by $\Gamma_{N}$ to the 
graded bimodule maps:

$$
\begin{aligned}
& \Gamma\left(\begin{array}{l|l}
\lambda+i_{X} & \oint \\
& \lambda
\end{array}\right):\left\{\begin{array}{l}
H_{\underline{k}^{+i}}\left\{1+k_{i-1}-k_{i+1}\right\} \longrightarrow H_{\underline{k}^{+i}}\left\{1+k_{i-1}-k_{i+1}\right\} \\
\xi_{i}^{\alpha} \mapsto \xi_{i}^{\alpha+1}
\end{array}\right.
\end{aligned}
$$

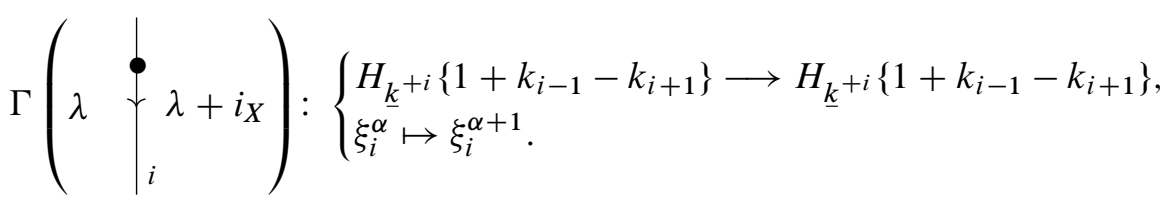

Note that these assignment are degree-preserving since these bimodule maps are degree 2 . maps:

The 2-morphisms $\nwarrow_{i, j, \lambda}$ and $\searrow_{\searrow_{i, j, \lambda}}$ are mapped by $\Gamma$ to the graded bimodule

$$
\begin{aligned}
& \Gamma\left(\succ_{i}^{\uparrow} \lambda\right): H_{+j \underline{k}^{+i}} \otimes_{H_{+j} \underline{k}} H_{\underline{k}^{+j}} \rightarrow H_{+i \underline{\underline{k}}}^{+j} \otimes_{H_{+i} \underline{\underline{k}}} H_{\underline{k}^{+i}},
\end{aligned}
$$

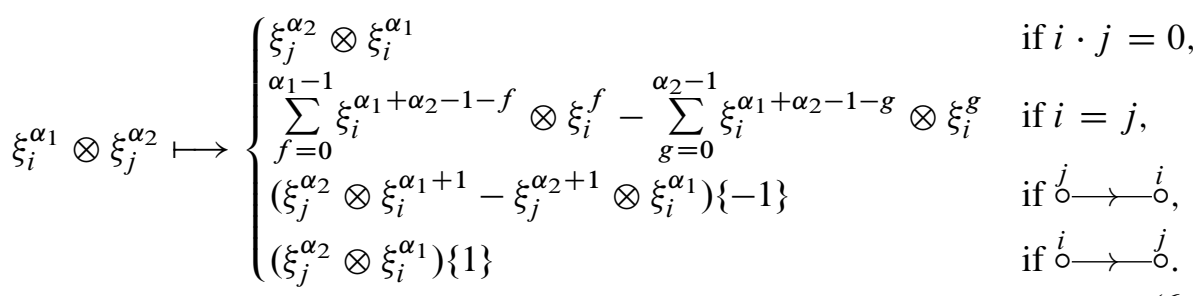

$$
\begin{aligned}
& \Gamma\left(\succ_{i \downarrow j}^{\lambda}\right): H_{-j \underline{k}^{-i}} \otimes_{H_{-j} \underline{k}} H_{\underline{k}^{-j}} \rightarrow H_{-i \underline{k}}^{-j} \otimes_{H_{-i} \underline{k}} H_{\underline{k}^{-i}},
\end{aligned}
$$

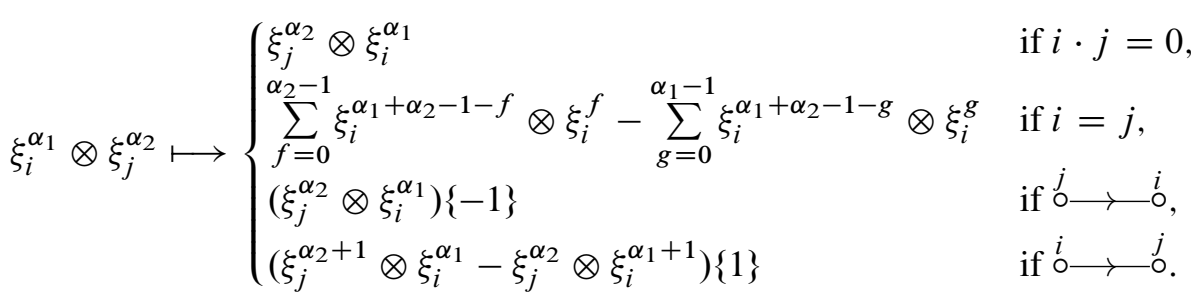

It is straightforward to see that these assignments define bimodule maps of degree $-i \cdot j$. In the case when $i=j$ the bimodule map is just the divided difference operator acting on $\xi_{i}$ and $\xi_{j}$. 
6.2. Checking the relations of $\boldsymbol{U}_{\rightarrow}$. In this section we show that the relations on the 2-morphisms of $U_{\rightarrow}^{*}$ are satisfied in $\mathbf{F l a g}_{N}^{*}$, thus establishing that $\Gamma$ is a graded additive $\mathbb{k}$-linear 2 -functor. From the definitions in the previous section it is clear that $\Gamma$ preserves the degrees associated to generators. For this reason, we often simplify our notation in this section by omitting the grading shifts $\{t\}$ when no confusion is likely to arise.

Proposition 6.3. $\Gamma$ preserves the $\mathfrak{s l}_{2}$-relations of $U_{\rightarrow}^{*}$.

Proof. The proof in [21] that the $\mathfrak{s} \Upsilon_{2}$-relations of $\mathcal{U}_{\rightarrow}^{*}$ are preserved by $\Gamma$ generalizes immediately with only minor refinements to grading shifts and summation indices. In [21] the condition that the dotted bubbles of degree zero are equal to one follows from [21, equation 8.1]. The proof of this proposition only makes use of (5.9) and (5.11)-(5.19). For example, to prove biadjointness we must show

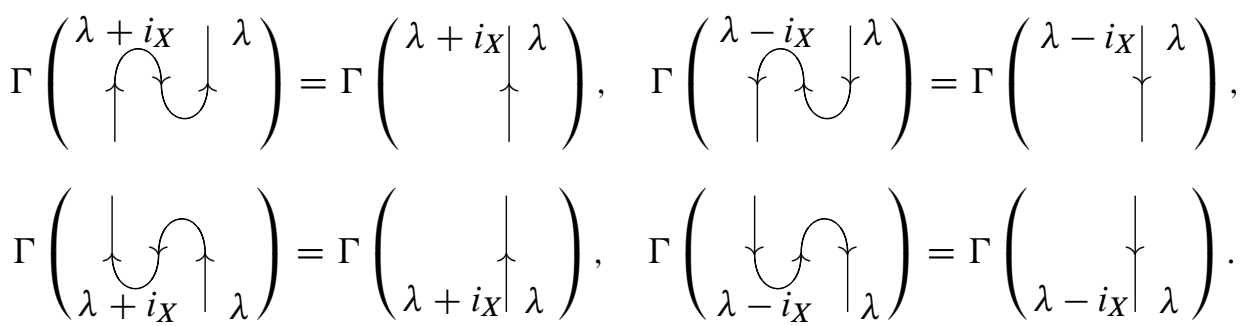

for all strings labelled by $i$. We will prove the first equality by computing the bimodules maps on elements $\xi_{i}^{\alpha} \in H_{\underline{k}}+i$.

$\Gamma\left(\stackrel{\lambda+i_{X}}{f} f^{\lambda}\right): \xi_{i}^{\alpha} \longmapsto \sum_{f=0}^{k_{i}-k_{i-1}}(-1)^{\alpha} i{ }_{i}^{i, \alpha-\left(k_{i}-k_{i-1}-f\right)} \uparrow{ }_{i, k_{i}-k_{i-1}-f}^{\lambda}$.

But

$$
\sum_{f=0}^{k_{i}-k_{i-1}}(-1)^{\alpha} i \stackrel{i, \alpha-\left(k_{i}-k_{i-1}-f\right)}{i, k_{i}-k_{i-1}-f} \stackrel{(5.17)}{=} \xi_{i}^{\alpha} .
$$

since the sum can be taken to $\alpha$ by removing terms that are equal to zero when $\alpha<k_{i}-k_{i-1}$, or by adding terms that are equal to zero if $\alpha>k_{i}-k_{i-1}$. The others are proven similarly.

\section{Lemma 6.4.}

$\Gamma(\overbrace{j}^{i} \overbrace{j}^{j} \lambda+i_{X})=\left\{\begin{array}{l}H_{\underline{k}^{+j}} \otimes_{H_{\underline{k}}} H_{\underline{k}^{+i}} \rightarrow H^{\alpha_{1+j} \underline{k}^{+i}} \otimes_{H_{+i+j \underline{k}}} H_{+i \underline{\underline{k}}}^{+j}, \\ \xi_{j}^{\alpha_{1}} \otimes \xi_{i}^{\alpha_{2}} \mapsto \xi_{i}^{\alpha_{2}} \otimes \xi_{j}^{\alpha_{1}},\end{array}\right.$ 


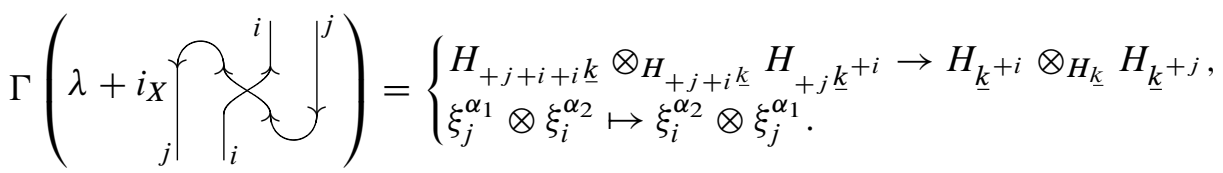

These bimodule maps have degree zero for all $i, j \in I$ and all weights $\lambda$.

Proof. We compute the bimodule maps directly using the definitions in the previous section. The case when $i=j$ appears in [21] so we will omit this case here. The map in (6.4), using (5.22) for the cup, is given by

$$
\begin{aligned}
& \Gamma(\overbrace{j}^{i} \overbrace{i}^{j} \lambda+i_{X}): \xi_{j}^{\alpha_{1}} \otimes \xi_{i}^{\alpha_{2}}
\end{aligned}
$$

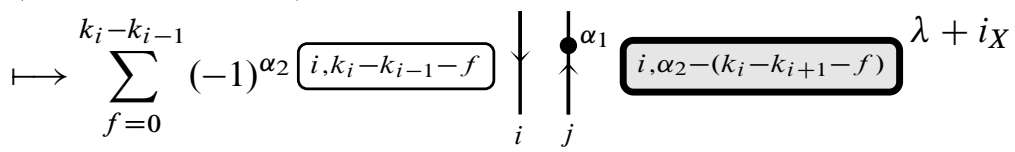

if $i \cdot j=0$ or $\stackrel{i}{\circ} \longrightarrow \stackrel{j}{\longrightarrow}$, and

$$
\begin{aligned}
& \left.\left.\sum_{f=0}^{k_{i}-k_{i-1}-1}(-1)^{\alpha_{2} \underbrace{}_{i, k_{i}-k_{i-1}-1-f}}\right|_{i}\right|_{j} ^{\alpha_{1}} \underbrace{\lambda+i_{X}}_{i, \alpha_{2}-\left(k_{i}-k_{i+1}-1-f\right)} \\
& -\left.\left.\sum_{f=0}^{k_{i}-k_{i-1}-1}(-1)^{\alpha_{2}-1}\right|_{i, k_{i}-k_{i-1}-1-f}\right|_{i, \alpha_{2}-\left(k_{i}-k_{i+1}-f\right)} ^{\alpha_{1}+1}+i_{X}{ }^{(6.6}
\end{aligned}
$$

if $\stackrel{j}{\circ} \longrightarrow \stackrel{i}{\circ}$. Careful calculation, keeping in mind the weights of each region, will show that these maps have degree zero. In both cases, the dual generators can slide across the line labelled $j$ via (5.19). After changing indices, equations (6.5) and (6.6) both become

$$
\sum_{f^{\prime}=0}^{\left(+{ }_{j} k\right)_{i}-\left(+{ }^{k}\right)_{i-1}}(-1)^{\alpha_{2}} i,\left.\left.f^{\prime}\right|_{i} ^{i, \alpha_{2}-f^{\prime}}\right|_{j} ^{\alpha_{1} \lambda+i_{X}}
$$

where $(+j k)_{i},(+j k)_{i-1}$ are the $(i-1)$-st and $i$-th terms in $+j \underline{k}$. By adding or removing terms that are equal to zero, depending on whether $\alpha_{2}$ is greater than or less than $\left(+{ }_{j} k\right)_{i}-\left(+{ }_{j} k\right)_{i-1}$, the above summation can be taken to $\alpha_{2}$, so that the dual version of (5.17) completes the proof.

Proposition 6.5. The equality

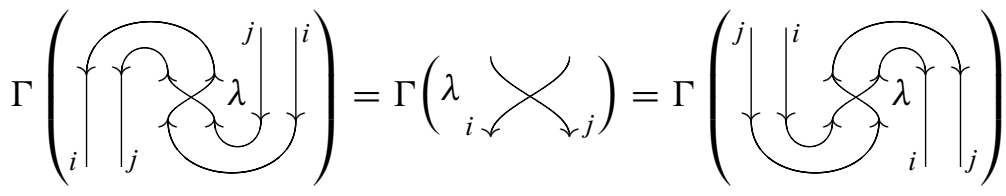


of graded bimodule maps holds in Flag $_{N}^{*}$ for all weights $\lambda$.

Proof. By direct calculation, using Lemma 6.4, we have that both the far left and right bimodule maps are given by

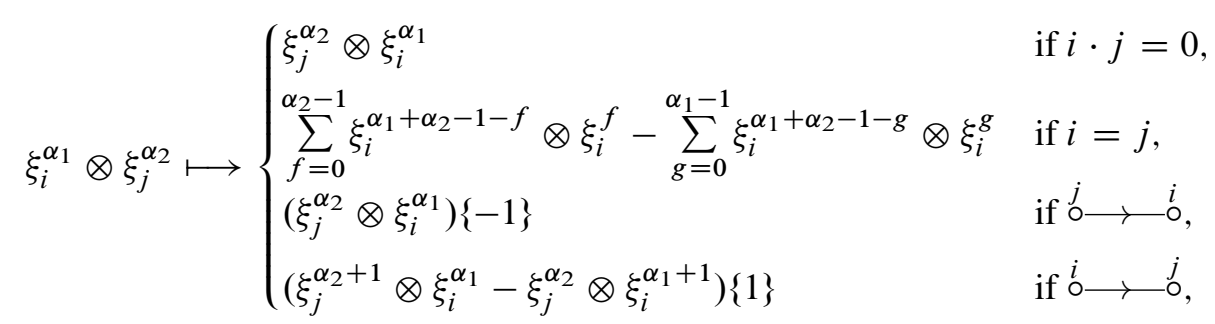

which agrees with $\Gamma\left(\searrow_{\searrow, j, \lambda+i_{X}+j_{X}}\right)$.

Proposition 6.6. The equalities

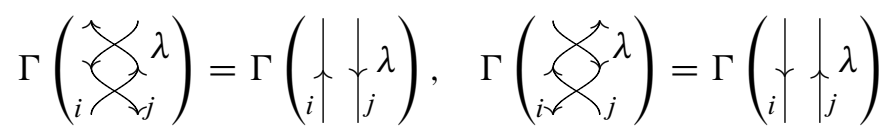

of graded bimodule maps hold in Flag $_{N}^{*}$ for all weights $\lambda$.

Proof. This follows immediately from Lemma 6.4.

Proposition 6.7. The equalities

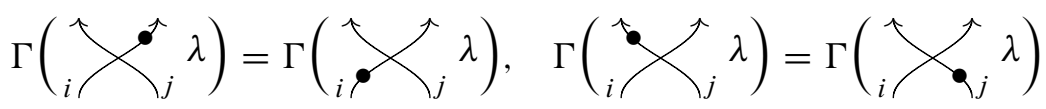

hold in Flag $_{N}^{*}$ for all weights $\lambda$.

Proof. Using the definition of the bimodule map $\Gamma\left(\nwarrow_{i, j, \lambda}\right)$ in (6.3) the proposition is easily verified.

Proposition 6.8. The equalities

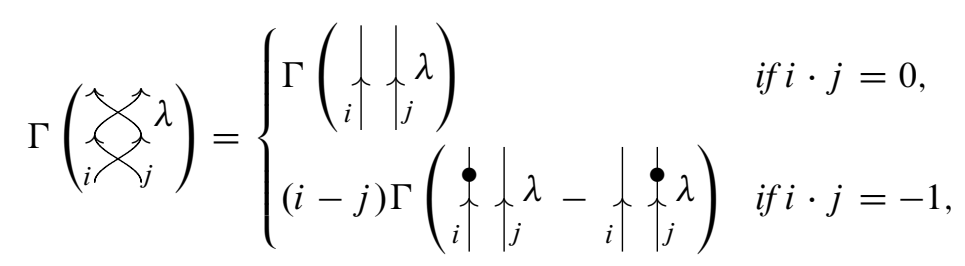




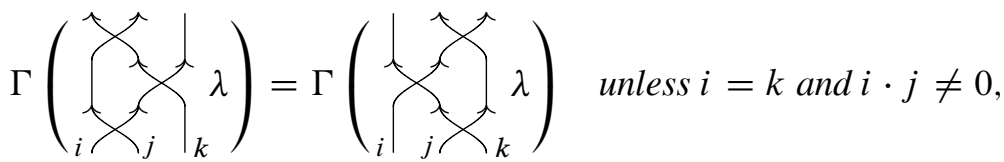

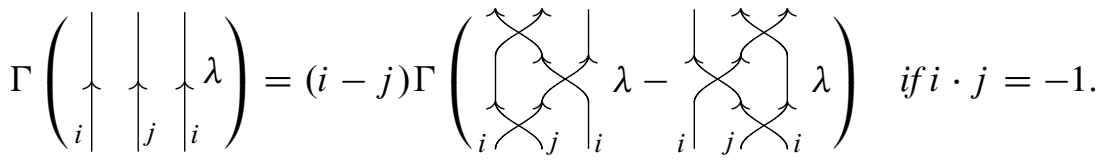

of bimodule maps hold in Flag $_{N}^{*}$ for all weights $\lambda$.

Proof. To prove the proposition we compute the bimodule maps in (6.7) on the elements of the form $\xi_{i}^{\alpha_{1}} \otimes \xi_{j}^{\alpha_{2}}$. Bimodule maps in (6.8) and (6.9) are computed on elements $\xi_{i}^{\alpha_{1}} \otimes \xi_{j}^{\alpha_{2}} \otimes \xi_{k}^{\alpha_{3}}$ and $\xi_{i}^{\alpha_{1}} \otimes \xi_{j}^{\alpha_{2}} \otimes \xi_{i}^{\alpha_{3}}$, respectively. The action on other elements in the cohomology rings is determined by the fact that the maps are bimodules morphisms.

The proof of these remaining relations is the same as the proof that rings $R(v)$ act on $\mathcal{P o}_{v}$ (notation as in [16]). Replacing the variables $x_{k}(\boldsymbol{i}) \in \mathcal{P} o \ell_{i}$ with the Chern classes of line bundles $\xi_{k}$ in the corresponding cohomology rings turns formulas in [16, Section 2.3] for the action of dots and crossings into formulas (6.2) and (6.3), with the signs taken into account.

Thus, we proved the following result:

Theorem 6.9. $\Gamma_{N}: \mathcal{U}_{\rightarrow}^{*} \rightarrow$ Flag $_{N}^{*}$ is a 2-functor and a 2-representation.

\subsection{Equivariant representation}

6.3.1. Reminders on equivariant cohomology. The GL $(N)$-equivariant cohomology of a point [11] is given by

$$
H_{\mathrm{GL}(N)}^{*}(\mathrm{pt})=H^{*}(\operatorname{Gr}(N, \infty))=\mathbb{k}\left[x_{1}, x_{2}, \ldots, x_{N}, y_{1}, y_{2}, \ldots\right] / I_{N, \infty}
$$

where $I_{N, \infty}$ is the ideal generated by the homogeneous terms in

$$
\left(1+x_{1} t+x_{2} t^{2}+\ldots x_{N} t^{N}\right)\left(1+y_{1} t+y_{2} t^{2}+\ldots y_{j} t^{j}+\cdots\right)=1 .
$$

Thus, $H_{\mathrm{GL}(N)}^{*}(\mathrm{pt})$ is isomorphic to the polynomial ring

$$
H_{\mathrm{GL}(N)}^{*}(\mathrm{pt}) \cong \mathbb{k}\left[x_{1}, x_{2}, \ldots, x_{N-1}, x_{N}\right]
$$

with $x_{i}$ in degree $2 i$.

Given a sequence $\underline{k}=\left(k_{0}, k_{1}, k_{2}, \ldots, k_{n}\right)$ with $0 \leq k_{1} \leq k_{2} \leq \cdots \leq k_{n}=N$, $\mathrm{GL}(N)$ acts transitively on $\mathrm{Fl}(\underline{k})$, so the equivariant cohomology of $\mathrm{Fl}(\underline{k})$ is

$$
H_{\mathrm{GL}(N)}^{*}(\mathrm{Fl}(\underline{k}))=H_{\mathrm{Stab}(\mathrm{pt})}^{*}(\mathrm{pt} \in \mathrm{Fl}(\underline{k}))
$$


where the stabilizer of a point $\left(0 \subset \mathbb{C}^{k_{1}} \subset \cdots \subset \mathbb{C}^{k_{n}}=C^{N}\right)$ in $F L(\underline{k})$ is the group of invertible block $\left(k_{1} \times\left(k_{2}-k_{1}\right) \times \cdots \times\left(N-k_{n-1}\right)\right)$ upper-triangular matrices. This group is contractible onto its subgroup $\mathrm{GL}\left(k_{1}\right) \times \mathrm{GL}\left(k_{2}-k_{1}\right) \times \cdots \times \mathrm{GL}\left(N-k_{n-1}\right)$. Hence,

$$
\begin{aligned}
H_{\underline{k}}^{G} & \cong H_{\mathrm{GL}\left(k_{1}\right) \times \mathrm{GL}\left(k_{2}-k_{1}\right) \times \cdots \times \mathrm{GL}\left(N-k_{n-1}\right)}^{*}(\mathrm{pt}) \\
& \cong \bigotimes_{j=1}^{n} H_{\mathrm{GL}\left(k_{j}-k_{j-1}\right)}^{*}(\mathrm{pt}) \\
& \cong \bigotimes_{j=1}^{n} \mathbb{k}\left[x(\underline{k})_{j, 1}, x(\underline{k})_{j, 2}, \ldots, x(\underline{k})_{j, k_{j}-k_{j-1}}\right]
\end{aligned}
$$

with $\operatorname{deg} x(\underline{k})_{j, \alpha}=2 \alpha$. Thus, the equivariant cohomology of $\operatorname{Fl}(\underline{k})$ has the same generators as the ordinary cohomology ring, but we do not mod out by the ideal $I_{\underline{k}, N}$.

The equivariant cohomology rings $H_{+i \underline{k}}^{G}$ and $H_{-i \underline{k}}^{G}$ of $\mathrm{Fl}(+i \underline{k})$ and $\mathrm{Fl}(-i \underline{k})$ can be similarly computed. They have the same generators as the ordinary cohomology rings $H_{+i \underline{k}}$ and $H_{-i \underline{k}}$, but no relations.

The equivariant cohomology $H_{\underline{k}^{+i}}^{G}$ of $\mathrm{Fl}\left(\underline{k}^{+i}\right)$ also has the same generators as the ordinary cohomology ring, with no relations. Using the forgetful maps $\mathrm{Fl}(\underline{k}) \leftarrow$ $\mathrm{Fl}\left(\underline{k}^{+i}\right) \rightarrow \mathrm{Fl}(+i \underline{k})$ we get inclusions

$$
\begin{aligned}
H_{\underline{k}}^{G} & \longmapsto H_{\underline{k}^{+i}}^{G}, \\
x(\underline{k})_{j, \alpha} & \longmapsto x\left(\underline{k}^{+i}\right)_{j, \alpha} \quad \text { for } j \neq i+1, \\
x(\underline{k})_{i+1, \alpha} & \longmapsto \xi_{i} \cdot x\left(\underline{k}^{+i}\right)_{i+1, \alpha-1}+x\left(\underline{k}^{+i}\right)_{i+1, \alpha},
\end{aligned}
$$

and

$$
\begin{aligned}
H_{+i \underline{k}}^{G} & \longmapsto H_{\underline{k}}^{G}, \\
x(+i \underline{k})_{j, \alpha} & \longmapsto x\left(\underline{k}^{+i}\right)_{j, \alpha} \quad \text { for } j \neq i, \\
x\left({ }_{+i}\right)_{i, \alpha} & \longmapsto \xi_{i} \cdot x\left(\underline{k}^{+i}\right)_{i, \alpha-1}+x\left(\underline{k}^{+i}\right)_{i, \alpha},
\end{aligned}
$$

making $H_{k^{+i}}^{G}$ a graded $\left(H_{+i \underline{k}}^{G}, H_{\underline{k}}\right)$-bimodule, just as in the non-equivariant case. Using these inclusions we introduce canonical generators of $H_{k^{+i}}^{G}$ given by identifying certain generators of $H_{\underline{k}}^{G}$ and $H_{+i \underline{k}}^{G}$ with their images in $H_{k^{+i}}^{G}$. Thus, we can identify $x(\underline{k})_{j, \alpha}$ and $x(+i \underline{k})_{j, \alpha}$ in $H_{\underline{k}^{+i}}^{G}$ when $j \neq i, i+1$, and

$$
\begin{aligned}
H_{\underline{k}^{+i}}^{G}=\bigotimes_{j \neq i+1} \mathbb{k}\left[x(\underline{k})_{j, 1} \ldots x(\underline{k})_{j, k_{j}-k_{j-1}}\right] \\
\\
\otimes \mathbb{k}\left[x(+i \underline{k})_{i+1,1}, \ldots, x(+i \underline{k})_{i+1, k_{i+1}-k_{i}-1}\right] \otimes \mathbb{k}\left[\xi_{i}\right],
\end{aligned}
$$


or equivalently

$$
\begin{aligned}
H_{\underline{k}}^{G i}=\bigotimes_{j \neq i} \mathbb{k}[ & \left.x(+i \underline{k})_{j, 1} \ldots x(+i \underline{k})_{j, k_{j}-k_{j-1}}\right] \\
& \otimes \mathbb{k}\left[x(\underline{k})_{i, 1}, \ldots, x(\underline{k})_{i, k_{i}-k_{i-1}}\right] \otimes \mathbb{k}\left[\xi_{i}\right] .
\end{aligned}
$$

The generators of $H_{\underline{k}}^{G}$ and $H_{+i \underline{k}}^{G}$ that are not mapped to canonical generators in $H_{\underline{k}^{+i}}^{G}$ can be expressed in terms of canonical generators as follows:

$$
\begin{aligned}
x(\underline{k})_{i+1, \alpha} & =\xi_{i} \cdot x(+i \underline{k})_{i+1, \alpha-1}+x(+i \underline{k})_{i+1, \alpha}, \\
x(+i \underline{k})_{i, \alpha} & =\xi_{i} \cdot x(\underline{k})_{i, \alpha-1}+x(\underline{k})_{i, \alpha}
\end{aligned}
$$

for all values of $\alpha$.

6.3.2. The 2-category $\mathbf{E q F l a g}_{N}$. The 2-category $\mathbf{E q F l a g}_{N}$ is the equivariant analog of $\operatorname{Flag}_{N}$.

Definition 6.10. The additive $\mathbb{k}$-linear 2-category $\mathbf{E q F l a g}_{N}$ is the idempotent completion inside of Bim of the 2-category consisting of:

- Objects: the graded rings $H_{k}^{G}$ for all $\underline{k}=\left(k_{0}, k_{1}, k_{2}, \ldots, k_{n}\right)$ with $0 \leq k_{1} \leq$ $k_{2} \leq \cdots \leq k_{n}=N$.

- Morphisms: generated by the graded $\left(H_{\underline{k}}^{G}, H_{\underline{k}}^{G}\right)$-bimodule $H_{\underline{k}}^{G}$, the graded $\left(H_{+i \underline{k}}^{G}, H_{\underline{k}}^{G}\right)$-bimodules $H_{\underline{k}^{+i}}^{G}$ and the graded $\left(H_{\underline{k}}^{G}, H_{+i \underline{k}}^{G}\right)$-bimodule $H_{\underline{k}+i}^{G}$ for all $i \in I$, together with their shifts $H_{\underline{k}}^{G}\{t\}, H_{k^{+i}}^{G}\{t\}$, and $H_{k^{+i}}^{G}\{t\}$ for $t \in \mathbb{Z}$. The bimodules $H_{\underline{k}}^{G}=H_{\underline{k}}^{G}\{0\}$ are the identity 1-morphisms. Thus, a morphism from $H_{\underline{k}}^{G}$ to $H_{i \underline{k}}^{G}$ is a finite direct sum of graded $\left(H_{j \underline{k}}^{G}, H_{\underline{k}}^{G}\right)$-bimodules of the form

$$
H_{\underline{k}}^{G}\{t\}:=H_{s_{2} s_{3} \ldots s_{m} \underline{k}^{s_{1}}}^{G} \otimes_{H_{s_{2} s_{3} \ldots s m \underline{k}}^{G}} \cdots \otimes_{H_{s_{m-1} s_{m} \underline{k}}^{G}} H_{s_{m} \underline{k}^{s_{m-1}}}^{G} \otimes_{H_{s_{m} \underline{k}}^{G}} H_{\underline{k}^{s m}}^{G}\{t\}
$$

for signed sequences $\boldsymbol{j}=s_{1} s_{2} \ldots s_{m}$ with $\boldsymbol{i}_{X}=\boldsymbol{j}_{X} \in X$.

- 2-morphisms: degree-preserving bimodule maps.

There is a graded additive subcategory $\mathbf{E q F l a g}_{N}^{*}$ of $\mathbf{B i m}{ }^{*}$ with the same objects and 1-morphisms as EqFlag $_{N}$, and

$$
\operatorname{EqFlag}_{N}^{*}\left(M_{1}, M_{2}\right):=\bigoplus_{t \in \mathbb{Z}} \operatorname{EqFlag}_{N}\left(M_{1}\{t\}, M_{2}\right)
$$


6.3.3. Equivariant representation $\Gamma_{N}^{G}$. A 2-representation of the 2-category $U^{*}\left(\mathfrak{s} \mathfrak{l}_{2}\right)=U_{\rightarrow}^{*}\left(\mathfrak{s} \mathfrak{l}_{2}\right)$ was constructed in [22] using equivariant cohomology of partial flag varieties. Here we extend that construction to the $\mathfrak{s l}_{n}$-case and define a 2-representation $\Gamma_{N}^{G}: U_{\rightarrow}^{*} \rightarrow \mathbf{E q F l a g}_{N}^{*}$.

The verification that $\Gamma_{N}$ is a 2-representation with the assignments given in Section 6.1 used only the relations (5.9) and (5.11)-(5.19), together with Lemma 5.4 and Corollary 5.5 which both follow from these relations.

To define the equivariant 2-representation $\Gamma_{N}^{G}$ ordinary cohomology rings are replaced by equivariant cohomology rings and bimodule maps associated to 2-morphisms are defined the same way, except that the dual generators $\overline{x(\underline{k})_{j, \alpha}}$ must be redefined. Set $\overline{x(\underline{k})_{j, 0}}=1$ and inductively define

$$
\overline{x(\underline{k})_{j, \alpha}}=-\sum_{f=1}^{\alpha} x(\underline{k})_{j, f} \overline{x(\underline{k})_{j, \alpha-f}} .
$$

For example,

$$
\begin{aligned}
& \overline{x(\underline{k})_{j, 1}}=-x(\underline{k})_{j, 1}, \\
& \overline{x(\underline{k})_{j, 2}}=-x(\underline{k})_{j, 1} \overline{x(\underline{k})_{j, 1}}-x(\underline{k})_{j, 2}=x(\underline{k})_{j, 1}^{2}-x(\underline{k})_{j, 2}, \\
& \overline{x(\underline{k})_{j, 3}}=-x(\underline{k})_{j, 1}^{3}+2 x(\underline{k})_{j, 1} x(\underline{k})_{j, 2}-x(\underline{k})_{j, 3} .
\end{aligned}
$$

In the nonequivariant cohomology rings the two definitions (5.3) and (6.12) of dual generators agree, but not in the equivariant cohomology ring.

Remark 6.11. We could have started with the definition of $\overline{x(\underline{k})_{j, \alpha}}$ given in (6.12) and used this definition to construct the non-equivariant representation in the previous section. After all, the two definitions (5.3) and (6.12) are equivalent in the nonequivariant cohomology ring. However, the recursive definition (6.12) makes it more cumbersome to calculate with in practice and that is why we used (5.3) to construct the non-equivariant representation.

Lemma 6.12. With $\overline{x(\underline{k})_{j, \alpha}}$ redefined as in (6.12) the relations (5.9) and (5.11)-(5.19) hold in the equivariant cohomology rings:

$$
\begin{aligned}
\sum_{f=0}^{\alpha} x(\underline{k})_{j, f} \overline{x(\underline{k})_{j, \alpha-f}} & =\delta_{\alpha, 0}, \\
x(\underline{k})_{j, \alpha} & =x(+i \underline{k})_{j, \alpha} \quad \text { for } j \neq i, i+1, \\
x(\underline{k})_{i+1, \alpha} & =\xi_{i} \cdot x(+i \underline{k})_{i+1, \alpha-1}+x(+i \underline{k})_{i+1, \alpha}, \\
x(+i \underline{k})_{i, \alpha} & =\xi_{i} \cdot x(\underline{k})_{i, \alpha-1}+x(\underline{k})_{i, \alpha}, \\
x(+i \underline{k})_{i+1, \alpha} & =\sum_{f=0}^{\alpha}(-1)^{f} \xi_{i}^{f} x(\underline{k})_{i+1, \alpha-f},
\end{aligned}
$$




$$
\begin{aligned}
& x(\underline{k})_{i, \alpha}=\sum_{f=0}^{\alpha} x(+i \underline{k})_{i, \alpha-f} \xi_{i}^{f}, \\
& (-1)^{\alpha} \xi_{i}^{\alpha}=\sum_{f=0}^{\alpha} x(+i \underline{k})_{i+1, \alpha-f} \overline{x(\underline{k})_{i+1, f}} \\
& =\sum_{g=0}^{\alpha} \overline{x(+i \underline{k})_{i \alpha-g}} x(\underline{k})_{i, g}, \\
& \overline{x(\underline{k})_{j, \alpha}}=\overline{x(+i \underline{k})_{j, \alpha}} \quad \text { if } j \neq i, i+1 \text {, } \\
& \overline{x(\underline{k})_{j, \alpha}}= \begin{cases}\overline{x(+i \underline{k})_{i, \alpha-1}} \xi_{i}+\overline{x(+i \underline{k})_{i, \alpha}} & \text { if } j=i, \\
\sum_{f=0}^{\alpha}(-1)^{\alpha} \overline{x(+i \underline{k})_{i+1, \alpha-f}} \xi_{i}^{f} & \text { if } j=i+1,\end{cases} \\
& \overline{x(+i \underline{k})_{j, \alpha}}= \begin{cases}\sum_{f=0}^{\alpha}(-1)^{\alpha} \overline{x(\underline{k})_{i, \alpha-f}} \xi_{i}^{f} & \text { if } j=i, \\
\overline{x(\underline{k})_{i+1, \alpha-1}} \xi_{i}+\overline{x(\underline{k})_{i+1, \alpha}} & \text { if } j=i+1,\end{cases}
\end{aligned}
$$

Proof. Equation (6.13) follows from the definition (6.12) of the dual elements $\overline{x(\underline{k})_{j, \alpha}}$. Equations (6.14)-(6.16) equate two images of noncanonical generators under the inclusions (6.10) and (6.11). Equations (6.17) and (6.18) follow from (6.15) and (6.16). The first equality in (6.19) is proven as follows:

$$
\begin{aligned}
(-1)^{\alpha} & \sum_{f=0}^{\alpha} x(+i \underline{k})_{i+1, \alpha-f} \overline{x(\underline{k})_{i+1, f}} \\
& \stackrel{(6.17)}{=} \sum_{f=0}^{\alpha} \sum_{g=0}^{\alpha-f}(-1)^{\alpha+g} \xi_{i}^{g} x(\underline{k})_{i+1, \alpha-f-g} \overline{x(\underline{k})_{i+1, f}} \\
& =\sum_{g=0}^{\alpha}(-1)^{\alpha+g} \xi_{i}^{g} \sum_{f=0}^{\alpha-g} x(\underline{k})_{i+1,(\alpha-g)-f} \overline{x(\underline{k})_{i+1, f}} \\
& \stackrel{(6.13)}{=} \sum_{g=0}^{\alpha}(-1)^{\alpha+g} \xi_{i}^{g} \delta_{\alpha-g, 0}=\xi_{i}^{\alpha} .
\end{aligned}
$$

The second equality above is just a re-indexing of the summation. The second equation in (6.19) is proven similarly.

Equation (6.20) follows from (6.14) and the definition of $\overline{x(\underline{k})_{j, \alpha}}$. Equations (6.21) and (6.22) follow from (6.15) and (6.16) and the definition of $\overline{x(\underline{k})_{j, \alpha}}$.

Theorem 6.13. $\Gamma_{N}^{G}: \mathcal{U}_{\rightarrow}^{*} \rightarrow$ EqFlag* $_{N}^{*}$ is a 2-representation.

Proof. It is clear that $\Gamma_{N}^{G}$ preserves the degree associated to 2-morphisms since the 2-representation $\Gamma_{N}$ does. The proof that $\Gamma_{N}^{G}$ preserves the relations in $U_{\rightarrow}^{*}$ can be 
copied line by line from the proof the $\Gamma_{N}$ preserves the relations. By Lemma 6.12 all identities used in the proof of Theorem 6.9 hold in $\mathbf{E q F l a g}{ }_{N}^{*}$ with the dual elements redefined according to (6.12).

Theorem 6.14. The 2-representations $\Gamma_{N}$ and $\Gamma_{N}^{G}$ categorify the irreducible representation $V_{N}$ of $\dot{\mathbf{U}}\left(\mathfrak{s} \mathfrak{l}_{n}\right)$ with highest weight $(N, 0, \ldots, 0)$.

Proof. Idempotent bimodule maps split in Bim, so by the universal property of the Karoubi envelope the additive 2-functors $\Gamma_{N}: U \rightarrow \operatorname{Flag}_{N}$ and $\Gamma_{N}^{G}: \mathcal{U} \rightarrow$ $\mathbf{E q F l a g}_{N}$, obtained using the isomorphism $\Sigma: \mathcal{U}^{*} \rightarrow \mathcal{U}_{\rightarrow}^{*}$ and restricting to degreepreserving 2-morphisms, extend to 2-representations of $\dot{u}$.

The rings $H_{k}$ and $H_{k}^{G}$ are graded local rings so that every projective module is free, and they both have (up to isomorphism and grading shift) a unique graded indecomposable projective module. The Grothendieck group of the category $\bigoplus_{\underline{k}} H_{\underline{k}}$-gmod (respectively $\bigoplus_{\underline{k}} H_{k}^{G}$-gmod) is a free $\mathbb{Z}\left[q, q^{-1}\right]$-module with basis elements $\left[H_{\underline{k}}\right]$ (respectively $\left[H_{\underline{k}}^{G}\right]$ ) over all $\underline{k}$, where $q^{i}$ acts by shifting the grading degree by $i$. Thus, we have

$$
K_{0}\left(\bigoplus_{\underline{k}} H_{\underline{k}}^{G}-\mathrm{gmod}\right) \cong K_{0}\left(\bigoplus_{\underline{k}} H_{\underline{k}}-\mathrm{gmod}\right) \cong{ }_{\mathcal{A}} V_{N}
$$

as $\mathbb{Z}\left[q, q^{-1}\right]$-modules, where the sums are over all sequences $0 \leq k_{1} \leq \cdots \leq k_{n}=N$ and ${ }_{\mathcal{A}} V_{N}$ is a representation of ${ }_{\mathcal{A}} \dot{\mathbf{U}}\left(\mathfrak{s} l_{n}\right)$, an integral form of the representation $V_{N}$ of $\dot{\mathbf{U}}\left(\mathfrak{s} \mathfrak{l}_{n}\right)$.

The bimodules $\Gamma\left(\mathbf{1}_{\lambda}\right), \Gamma\left(\mathcal{E}_{+i} \mathbf{1}_{\lambda}\right), \Gamma\left(\mathcal{E}_{-i} \mathbf{1}_{\lambda}\right)$ (or equivalently $\Gamma^{G}\left(\mathbf{1}_{\lambda}\right), \Gamma^{G}\left(\mathcal{E}_{+i} \mathbf{1}_{\lambda}\right)$ and $\left.\Gamma^{G}\left(\mathcal{E}_{-i} \mathbf{1}_{\lambda}\right)\right)$ induce functors on the graded module categories given by tensoring with these bimodules. The functors

$$
\begin{aligned}
\mathbf{1}_{\lambda} & :=H_{k} \otimes_{H_{\underline{k}}}-: H_{\underline{k}}-\operatorname{gmod} \rightarrow H_{\underline{k}}-\operatorname{gmod} \\
\mathcal{E}_{i} \mathbf{1}_{\lambda}: & =H_{\underline{k}^{+i}} \otimes_{H_{\underline{k}}}-\left\{1+k_{i-1}-k_{i+1}\right\}: H_{\underline{k}}-\operatorname{gmod} \rightarrow H_{+i \underline{k}-\operatorname{gmod}} \\
\mathcal{F}_{i} \mathbf{1}_{\lambda+i_{X}}: & =H_{\underline{k}^{+i}} \otimes_{H_{+i} \underline{k}}-\left\{-k_{i}\right\}: H_{+i \underline{k}}-\operatorname{gmod} \rightarrow H_{\underline{k}^{-g m o d}}
\end{aligned}
$$

have both left and right adjoints and commute with the shift functor, so they induce $\mathbb{Z}\left[q, q^{-1}\right]$-module maps on Grothendieck groups. Furthermore, the 2 -functor $\Gamma_{N}$ respects the relations of $\dot{U}$, so by Propositions 3.24-3.26 these functors satisfy relations lifting those of $\dot{\mathbf{U}}$.

\subsection{Nondegeneracy of $U_{\rightarrow}\left(\mathfrak{s} \mathfrak{l}_{n}\right)$}

Lemma 6.15. The surjective graded $\mathbb{k}$-algebra homomorphism

$$
\Pi_{\lambda} \rightarrow U^{*}\left(\mathbf{1}_{\lambda}, \mathbf{1}_{\lambda}\right) \cong U_{\rightarrow}^{*}\left(\mathbf{1}_{\lambda}, \mathbf{1}_{\lambda}\right)
$$

of Proposition 3.6 is an isomorphism. 
Proof. Injectivity is established by showing that for each $M \in \mathbb{N}$ there exists some large $N$ such that the images of bubble monomials in $\mathcal{U}_{\rightarrow}^{*}\left(\mathbf{1}_{\lambda}, \mathbf{1}_{\lambda}\right)$ in variables of degree less than $M$ act by linearly independent operators on $H_{\underline{k}}^{G}$ for $\lambda=\lambda(\underline{k})$, under the 2-functor $\Gamma_{N}^{G}$. By a direct calculation

$$
\begin{gathered}
\Gamma^{G}(\underbrace{i}_{\lambda_{i}-1+\alpha}): 1 \longrightarrow(-1)^{\alpha} \sum_{f=0}^{\lambda}{ }^{\min \left(\alpha, k_{i+1}-k_{i}\right)} \overline{x(\underline{k})_{i, \alpha-f}} x(\underline{k})_{i+1, f}, \\
\left.\Gamma_{-\lambda_{i}-1+\beta}^{\lambda}\right): 1 \longrightarrow(-1)^{\beta} \sum_{g=0}^{\min \left(\beta, k_{i}-k_{i-1}\right)} \overline{x(\underline{k})_{i+1, \alpha-g}} x(\underline{k})_{i, g},
\end{gathered}
$$

as bimodule endomorphisms of $H_{\underline{k}}^{G}$. After expanding the $\overline{x(\underline{k})_{j, \alpha}}$ using (6.12) we have

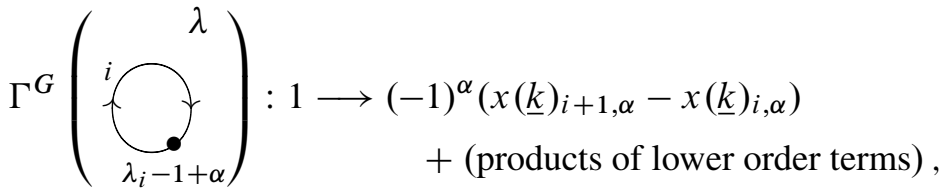

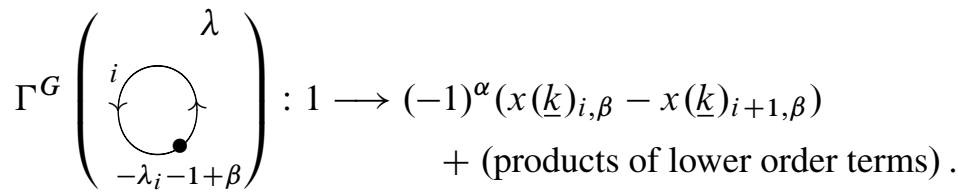

Only one orientation for dotted bubbles labelled by vertex $i$ is allowed in a bubble monomial in $\Pi_{\lambda}$, see (3.19). The image under $\Gamma^{G}$ of a bubble monomial is composed of products of bimodule maps of the above form where, in the equivariant cohomology ring, sums of products of element of the form $\left(x(\underline{k})_{i+1, \alpha}-x(\underline{k})_{i, \alpha}\right)$, respectively $\left(x(\underline{k})_{i, \beta}-x(\underline{k})_{i+1, \beta}\right)$ are independent provided $\alpha, \beta<k_{i}-k_{i-1}$ and $\alpha, \beta \leq k_{i+1}-k_{i}$ so that $\left(x(\underline{k})_{i+1, \alpha}-x(\underline{k})_{i+1, \alpha}\right)$, respectively $\left(x(\underline{k})_{i, \beta}-x(\underline{k})_{i+1, \beta}\right)$, is nonzero. By taking $N$ large, we can ensure this condition is satisfied for any fixed $M$. Hence, images of bubble monomials in $\mathcal{U}_{\rightarrow}^{*}\left(\mathbf{1}_{\lambda}, \mathbf{1}_{\lambda}\right)$ are independent.

Lemma 6.16. There is an isomorphism of graded $\mathbb{k}$-algebras,

$$
\begin{gathered}
\iota_{\lambda}^{\prime}: R(v) \otimes \Pi_{\lambda} \longrightarrow U_{\rightarrow}^{*}\left(\mathcal{E}_{\nu} \mathbf{1}_{\lambda}, \mathcal{E}_{\nu} \mathbf{1}_{\lambda}\right), \\
D \otimes D_{\pi} \longmapsto \iota_{\lambda}(D) \cdot D_{\pi},
\end{gathered}
$$

with $\iota_{\lambda}$ given by (4.3) and $D_{\pi}$ a bubble monomial in $\Pi_{\lambda}$.

Proof. Surjectivity of $\iota_{\lambda}^{\prime}$ follows from Lemma 3.9 and the isomorphism $\Sigma$ from Section 4.2.1. Injectivity of $\iota_{\lambda}^{\prime}$ is established by showing that for each $M \in \mathbb{N}$ there 
exists some large $N$ such that degree $M$ elements $\iota_{\lambda}(D) \cdot D_{\pi}$, as $D$ and $D_{\pi}$ run over a basis of $R(v)$, respectively $\Pi_{\lambda}$, act by linear independent operators under the 2representation $\Gamma_{N}^{G}$. The 2-functor $\Gamma^{G}$ must map horizontal composites to horizontal composites (given by tensor products) in $\mathbf{E q F l a g}{ }_{N}^{*}$, so that

$$
\Gamma^{G}\left(\iota_{\lambda}(D) \cdot D_{\pi}\right)=\Gamma^{G}\left(\iota_{\lambda}(D)\right) \otimes_{H_{\underline{k}}^{G}} \Gamma^{G}\left(D_{\pi}\right):=f_{D} \otimes_{H_{\underline{k}}^{G}} g_{D_{\pi}}
$$

for bimodule maps $g_{D_{\pi}}: H_{\underline{k}}^{G} \rightarrow H_{\underline{k}}^{G}$ and $f_{D}: H_{\underline{k}^{i}}^{G} \rightarrow H_{\underline{k}^{j}}^{G}$ for some $\boldsymbol{i}, \boldsymbol{j} \in \operatorname{Seq}(\nu)$.

Let

$$
\operatorname{Po\ell }_{v}(\xi):=\bigoplus_{i \in \operatorname{Seq}(v)} \operatorname{Po}_{\boldsymbol{i}}, \quad \operatorname{Po}_{\boldsymbol{i}}=\mathbb{k}\left[\xi_{\boldsymbol{i}_{1}}, \xi_{\boldsymbol{i}_{2}}, \ldots, \xi_{\boldsymbol{i}_{m}}\right], \quad m=|v|
$$

For large enough $N$, the bimodule $\Gamma^{G}\left(\mathcal{E}_{\nu} \mathbf{1}_{\lambda}\right)$ contains $\mathcal{P} o \ell_{\nu}(\xi)$ as a subspace. From the definition of $\iota_{\lambda}$ (see (4.3)), together with the definitions of the bimodule maps associated to $R(v)$ generators (see Section 6.1.2), it is clear that the action on $\operatorname{Po}_{v}(\xi)$ given by the 2 -functor $\Gamma^{G}$ coincides with the action of $R(v)$ on $\mathcal{P} o \ell_{v}$ defined in [16]. In particular, bimodule maps $f_{D}$ corresponding to basis elements of $R(v)$ must act by linear independent operators (see [16, proof of Theorem 2.5]) on the subspace $\operatorname{Po}_{\nu}(\xi)$ of $\Gamma^{G}\left(\mathcal{E}_{\nu} \mathbf{1}_{\lambda}\right)$. Furthermore, from the definitions of bimodule maps associated to $R(v)$ generators it is also clear that $f_{D}$ fixes all other generators of $\Gamma^{G}\left(\varepsilon_{\nu} \mathbf{1}_{\lambda}\right)$.

By Lemma 6.15, for large $N$ the bimodule maps $g_{D_{\pi}}$ act by linearly independent operators on $H_{\underline{k}}^{G}$. Write

$$
\pi_{i, \alpha}:= \begin{cases}\langle i, \lambda\rangle_{-1+\alpha}^{i} \lambda & \text { for }\langle i, \lambda\rangle \geq 0, \\ \underbrace{i}_{-\langle i, \lambda\rangle}\rangle_{-1+\alpha}^{\lambda} & \text { for }\langle i, \lambda\rangle<0 .\end{cases}
$$

and let $D_{\pi}=\pi_{\ell_{1}, \alpha_{1}} \pi_{\ell_{2}, \alpha_{2}} \ldots \pi_{\ell_{r}, \alpha_{r}}$. Then $D_{\pi}$ acts on $H_{\underline{k}^{i}}^{G} \cong H_{\underline{k}^{i}}^{G} \otimes_{H_{\underline{k}}^{G}} H_{\underline{k}}^{G}$ via $\Gamma^{G}\left(\operatorname{Id}_{\mathbf{1}_{\lambda}} \cdot D_{\pi}\right)=1 \otimes_{H_{\underline{k}}^{G}} g_{D_{\pi}}$. From (6.23) we have

$$
\begin{array}{r}
1 \otimes_{H_{\underline{k}}^{G}} g_{D_{\pi}}: 1 \otimes 1 \longmapsto\left(\sum_{f_{1}=0}^{\alpha_{1}} x(\underline{k})_{\ell_{1}+1, f_{1}} \overline{x(\underline{k})_{\ell_{1}, \alpha_{1}-f_{1}}}\right) \\
\cdots\left(\sum_{f_{r}=0}^{\alpha_{r}} x(\underline{k})_{\ell_{1}+1, f_{r}} \overline{x(k) \ell_{r}, \alpha_{r}-f_{r}}\right)
\end{array}
$$

in $H_{k^{i}}^{G}$. After expanding the $\overline{x(\underline{k})_{\ell, \alpha}}$ using their definition (6.12), we have an expression for the action of $g_{D_{\pi}}$ on $H_{\underline{k}^{i}}^{G}$ strictly in terms of variables $x(\underline{k})_{\ell, \alpha}$. 
The bimodule maps $f_{D} \otimes_{H_{k}^{G}} g_{D_{\pi}}$ are linearly independent operators since the bimodule maps $f_{D}$ and $g_{D_{\pi}}$ are separately independent and act on algebraically independent generators of $H_{k^{i}}^{G}$ :

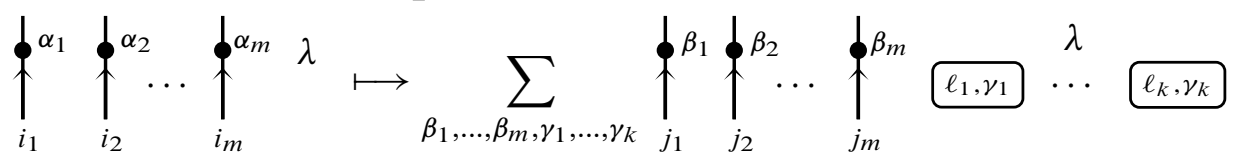

where the sum over the $\beta_{a}$ 's is determined by the action of $f_{D}$ on $\xi_{1}^{\alpha_{1}} \otimes \cdots \otimes \xi_{m}^{\alpha_{m}}$, and the sum over the $x(\underline{k})_{\ell_{b}, \gamma_{b}}$, represented by labelled boxes on the far right, is determined from (6.24) by expanding the $\overline{x(k)}_{j, \alpha}$. In particular, $f_{D}$ fixes variables $x(\underline{k})_{\ell, \alpha}$ represented by labelled boxes on the far right and $g_{D}$ acts only on such boxes.

This concludes the proof of Theorem 1.3 stated in the introduction, so that

$$
\gamma:{ }_{A} \dot{\mathbf{U}}\left(\mathfrak{s} \mathfrak{l}_{n}\right) \longrightarrow K_{0}\left(\dot{U}\left(\mathfrak{s}_{n}\right)\right)
$$

is an isomorphism. Proposition 3.28 allows us to view 2-functors $\tilde{\psi}, \tilde{\omega}, \tilde{\sigma}$, and $\tilde{\tau}$ as categorifications of symmetries $\psi, \omega, \sigma$, and $\tau$ of ${ }_{\mathcal{A}} \dot{\mathbf{U}}\left(\mathfrak{s l}_{n}\right)$. The graded homs between 1-morphisms in $\dot{U}\left(\mathfrak{s} \mathfrak{l}_{n}\right)$ categorify the semilinear form $\langle,\rangle_{\pi}$ given by (3.28).

\section{References}

[1] S. Ariki, On the decomposition numbers of the Hecke algebra of $G(m, 1, n)$. J. Math. Kyoto Univ. 36 (1996), 789-808. MR 1443748 Zbl 0888.20011

[2] A. A. Beĭlinson, G. Lusztig, and R. MacPherson, A geometric setting for the quantum deformation of $G L_{n}$. Duke Math. J. 61 (1990), 655-677. MR 1074310 Zbl 0713.17012

[3] J. Bernstein, I. Frenkel, and M. Khovanov, A categorification of the Temperley-Lieb algebra and Schur quotients of $U\left(\mathfrak{s I}_{2}\right)$ via projective and Zuckerman functors. Selecta Math. (N.S.) 5 (1999), 199-241. MR 1714141 Zbl 0981.17001

[4] F. Borceux, Handbook of categorical algebra, Vol. 1: Basic category theory. Encyclopedia Math. Appl. 50, Cambridge University Press, Cambridge 1994. MR 1291599 Zbl 0803.18001

[5] S. Cautis and J. Kamnitzer, Knot homology via derived categories of coherent sheaves, I: The sl(2)-case. Duke Math. J. 142 (2008), 511-588. MR 2411561 Zbl 1145.14016

[6] S. Cautis and J. Kamnitzer, Knot homology via derived categories of coherent sheaves II, $s l_{m}$ case. Invent. Math. 174 (2008), 165-232. MR 2430980 Zbl 05343986

[7] J. Chuang and R. Rouquier, Derived equivalences for symmetric groups and $\mathfrak{s}_{2}$ categorification. Ann. of Math. (2) 167 (2008), 245-298. MR 2373155 Zbl 1144.20001

[8] L. Crane and I. B. Frenkel, Four-dimensional topological quantum field theory, Hopf categories, and the canonical bases. J. Math. Phys. 35 (1994), 5136-5154. MR 1295461 Zbl 0892.57014 
[9] V. G. Drinfel'd, Quantum groups. In Proc. Internat. Congr. Math. (Berkeley, Calif., 1986), Vol. 1, Amer. Math. Soc., Providence, RI, 1987, 798-820. MR 0934283 Zbl 0667.16003

[10] I. Frenkel, M. Khovanov, and C. Stroppel, A categorification of finite-dimensional irreducible representations of quantum $\mathfrak{s l}_{2}$ and their tensor products. Selecta Math. (N.S.) 12 (2006), 379-431. MR 2305608 Zbl 05189561

[11] W. Fulton, Equivariant cohomology in algebraic geometry. Eilenberg lectures, Columbia University, Notes by Dave Anderson, 2007. http://www.math.washington.edu/ dandersn/eilenberg/

[12] N. Ganter and M. Kapranov, Representation and character theory in 2-categories. Adv. Math. 217 (2008), 2268-2300. MR 2388094 Zbl 1136.18001

[13] M. Jimbo, A $q$-difference analogue of $U(\mathrm{~g})$ and the Yang-Baxter equation. Lett. Math. Phys. 10 (1985), 63-69. MR 797001 Zbl 0587.17004

[14] M. Kashiwara, Global crystal bases of quantum groups. Duke Math. J. 69 (1993), 455-485. MR 1203234 Zbl 0774.17018

[15] M. Khovanov, A functor-valued invariant of tangles. Algebr. Geom. Topol. 2 (2002), 665-741. MR 1928174 Zbl 1002.57006

[16] M. Khovanov and A. Lauda, A diagrammatic approach to categorification of quantum groups I. Preprint 2008. arXiv:0803.4121 [math.QA]

[17] M. Khovanov and A. Lauda, A diagrammatic approach to categorification of quantum groups II. Preprint 2008. arXiv:0804.2080 [math.QA]

[18] M. Khovanov and L. Rozansky, Matrix factorizations and link homology. Fund. Math. 199 (2008), 1-91. MR 2391017 Zbl 1145.57009

[19] A. Kleshchev, Linear and projective representations of symmetric groups. Cambridge Tracts in Math. 163, Cambridge University Press, Cambridge 2005. MR 2165457 Zbl 1080.20011

[20] P. B. Kronheimer and H. Nakajima, Yang-Mills instantons on ALE gravitational instantons. Math. Ann. 288 (1990), 263-307. MR 1075769 Zbl 0694.53025

[21] A. D. Lauda, A categorification of quantum sl(2). Preprint 2008. arXiv:0803.3652 [math.QA]

[22] A. D. Lauda, Categorified quantum sl(2) and equivariant cohomology of iterated flag varieties. Preprint 2008. arXiv:0803.3848 [math.QA]

[23] G. Lusztig, Canonical bases in tensor products. Proc. Nat. Acad. Sci. U.S.A. 89 (1992), 8177-8179. MR 1180036 Zbl 0760.17011 http://www.pnas.org/content/89/17/8177.full.pdf+html

[24] G. Lusztig, Introduction to quantum groups. Progr. Math. 110, Birkhäuser, Boston 1993. MR 1227098 Zbl 0788.17010

[25] G. Lusztig, Canonical bases and Hall algebras. In Representation theories and algebraic geometry (Montreal, PQ, 1997), NATO Adv. Sci. Inst. Ser. C Math. Phys. Sci. 514, Kluwer Acad. Publ., Dordrecht 1998, 365-399. MR 1653038 Zbl 0934.17010

[26] H. Nakajima, Instantons on ALE spaces, quiver varieties, and Kac-Moody algebras. Duke Math. J. 76 (1994), 365-416. MR 1302318 Zbl 0826.17026 
[27] M. Reineke, Monomials in canonical bases of quantum groups and quadratic forms. $J$. Pure Appl. Algebra 157 (2001), 301-309. MR 1812057 Zbl 1022.17011

[28] J. Rosenberg, Algebraic K-theory and its applications. Grad. Texts in Math. 147, Springer-Verlag, New York 1994. MR 1282290 Zbl 0801.19001

[29] R. Rouquier, Higher representation theory. Talk at IAS, March 2008.

[30] R. Rouquier, Higher representations of Kac-Moody algebras. Work in progress.

[31] J. Sussan, Category $\mathcal{O}$ and $\mathfrak{s l}_{k}$ link invariants. Preprint 2007. arXiv:math/0701045 [math.QA]

[32] C. Weibel, The K-book: An introduction to algebraic K-theory. http://www.math.rutgers.edu/ weibel/Kbook.html

[33] H. Zheng. Categorification of integrable representations of quantum groups. Preprint 2008. arXiv:0803.3668 [math.RT]

Received July 5, 2009

M. Khovanov, Columbia University, New York, NY 10027, USA

E-mail: khovanov@math.columbia.edu

A. D. Lauda, Columbia University, New York, NY 10027, USA

E-mail: lauda@math.columbia.edu 